\title{
A Categorization of Power Measurement and Power Consumption Models in the Telco Cloud
}

\section{Etienne-Victor Depasquale ( $\nabla$ edepa@ieee.org )}

University of Malta https://orcid.org/0000-0003-3642-6305

\section{Humaira Abdul Salam}

Deutsches Elektronen-Synchrotron

Franco Davoli

University of Genoa: Universita degli Studi di Genova

\section{Research Article}

Keywords: Virtualization, power consumption, power models, power meters, energy-aware algorithms

Posted Date: October 5th, 2021

DOI: https://doi.org/10.21203/rs.3.rs-782848/v1

License: (c) (1) This work is licensed under a Creative Commons Attribution 4.0 International License. Read Full License 


\title{
A Categorization of Power Measurement and : Power Consumption Models in the Telco Cloud :
}

\author{
Etienne-Victor Depasquale ${ }^{7}$, Humaira Abdul Salam ${ }^{2}$ and Franco Davoli ${ }^{3}$ \\ 8

${ }^{1}$ Department of Communications and Computer Engineering, University of Malta, MSD2080 Msida, Malta

Full list of author information is available at the end of the article

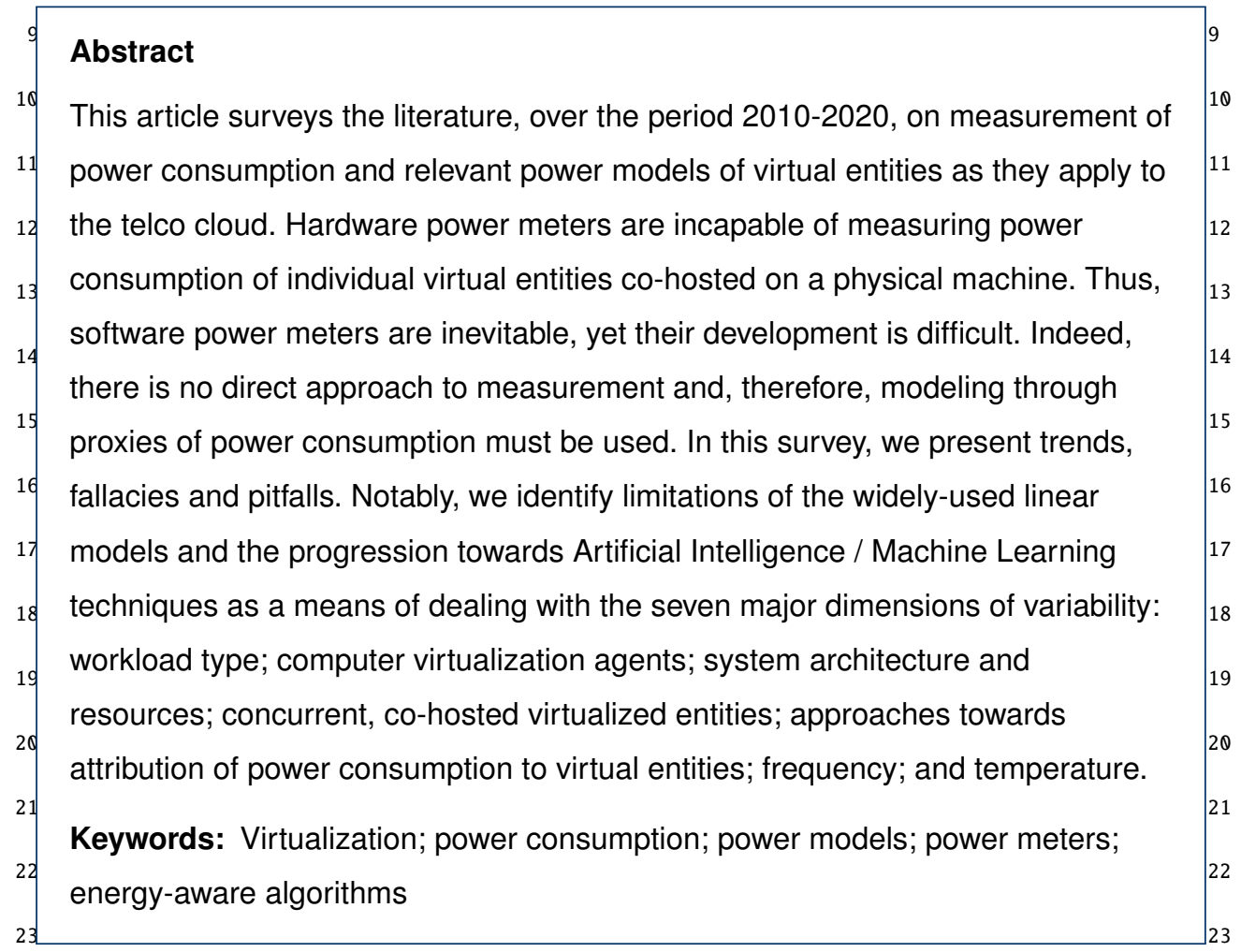

${ }^{26} \boldsymbol{1}$ Introduction 26

${ }^{27}$ Accuracy in power and energy measurements is necessary to devise energy- and/or power- ${ }^{27}$ ${ }^{28}$ efficient operations. It is also essential for billing in multi-tenant environments, so that the ${ }^{28}$ ${ }^{29}$ Infrastructure Provider (IPr) can charge customers the fair amount for the resources (in- ${ }^{29}$ ${ }^{30}$ cluding energy) they consume. Precise measurement of a virtual entity's (VE, i.e. virtual ${ }^{30}$ ${ }^{31}$ machine (VMs) or container) power consumption is difficult, since host power meter mea- ${ }^{31}$ ${ }^{32}$ surements cannot be related directly to it. Moreover, power consumption of a VE varies ${ }^{32}$ ${ }^{33}$ with its hosting machine. Therefore, for VEs, accurate measurement is predicated upon ${ }^{33}$ 
${ }^{1}$ precise modelling of energy- and/or power consumption. Before proceeding to the scope ${ }^{1}$

${ }^{2}$ of our survey, we pose three salient questions that frame our work.

2

3

${ }_{41.1}$ Why should energy consumption be a topic of interest?

${ }_{5}$ Energy efficiency in the Internet (and in computing and telecommunication networks in 5 ${ }_{6}$ general) has become a significant problem, which has received increasing attention since 6 7the early years 2000 (see, e.g., [1] [2] [3] and references therein), starting from cloud com-7 sputing infrastructures, and then extending to mobile and fixed networks. Indeed, it has been 8 gshown that the smaller data centres, within which telecommunications points of presence 9 ${ }_{10}(\mathrm{PoPs})$ may be classified, represent around $95 \%$ of the United States' data center energy 10 11 use [4]. Furthermore, this use is comparatively inefficient when compared with that of the 11 12hyperscale server farms (the remaining 5\%). 12 13

1.2 What is the underlying cause?

14 ${ }_{15}$ Traffic growth is one of the causes of increased energy consumption. Table 1 shows the ${ }_{15}$ ${ }_{16}{ }^{\text {consistency with which Cisco's Visual Networking Index (VNI) has been predicting heavy }}{ }_{16}$ ${ }_{17}$ growth in traffic exchanged over the access network by both businesses and consumers ${ }_{17}$ with:

19

1 endpoints over managed networks and

18

2 endpoints over unmanaged networks ("Internet traffic").

19 ${ }_{21}$ Note that the figures refer to compound annual growth rate (CAGR); they do not refer to ${ }_{21}$ ${ }_{22}$ the percentage share of total traffic. The key observation lies in the realization that, year ${ }_{22}$ ${ }_{23}$ after year, significant (heavy, in the case of mobile data) growth is persistently predicted. ${ }_{23}$

${ }^{24}$ Table 1: Compound Annual Growth Rate (CAGR) reported in Cisco's VNI over four con- ${ }^{24}$ 25

\begin{tabular}{|c|c|c|c|c|}
\hline Period & Fixed Internet Traffic & Managed IP Traffic & Mobile Data & 26 \\
\hline 2014-2019 [5] & 23 & 13 & 57 & 27 \\
\hline $2015-2020$ [6] & 21 & 11 & 53 & \\
\hline 2016-2021 [7] & 23 & 13 & 46 & 28 \\
\hline $2017-2022$ [8] & 26 & 11 & 46 & \\
\hline
\end{tabular}

30 This is corroborated by several other researchers, with perspectives varying from traffic ${ }^{30}$ ${ }^{31}$ at the access segment to traffic in transit between Internet Service Providers (ISPs) [9] [10] ${ }^{31}$ 32 [11]. Cisco [5] and Sandvine [12][13] identify "video traffic" and "real-time entertainment" ${ }^{33}$ as the drivers of this growth. The latest edition of Sandvine's Global Internet Phenomena ${ }^{33}$ 
${ }^{1}$ report [14] dedicates its executive summary exclusively to video traffic; the report shows ${ }^{1}$

${ }^{2}$ video as consuming $60 \%$ of downstream traffic, a further $2 \%$ increase over 2018 . Alcatel- ${ }^{2}$

${ }^{3}$ Lucent Bell Labs [10] observed that growth in the metro-core due to video traffic exceeds ${ }^{3}$

${ }^{4}$ growth in video traffic crossing the long-haul core. Indeed, it is widely recognized that ${ }^{4}$

${ }^{5}$ video is the prime driver of this growth in traffic and attendant energy consumption and ${ }^{5}$

${ }^{6}$ research has been addressing this problem [15-26].

${ }^{8} 1.3$ How is energy consumption being tackled? 8

${ }^{9}$ From these works, development of cache architectures emerges as an important approach ${ }^{9}$

${ }^{10}$ to controlling energy consumption, but other mechanisms exist and yet more are emerg ${ }^{10}$

${ }^{11}$ ing within the general thrust towards "future networks" (ITU-T Y.3001 [27]). Networks ${ }^{11}$

${ }^{12}$ are evolving into flexible and programmable "softwarized" virtualized infrastructures, and ${ }^{12}$

${ }^{13}$ strongly integrated paradigms as in $5 \mathrm{G}[28,29]$ rewrite the research agenda. We may now ${ }^{13}$

${ }^{14}$ distinguish between radical and reformist approaches. Reformist approaches seek to im- ${ }^{14}$

${ }^{15}$ prove the caching of content and are characterized by investigation of the reduction of the ${ }^{15}$

${ }^{16}$ length of the path between source and destination of IP traffic. Radical approaches employ ${ }^{16}$

${ }^{17}$ the dynamic and reactive control afforded by the softwarized, virtualized infrastructures. ${ }^{17}$

${ }^{18}$ Radical methods are enabled by standardized architectures (e.g. [30]) that equip the control ${ }^{18}$

${ }^{19}$ plane with uniform interfaces that exploit extant and emerging green capabilities. Signif- ${ }^{19}$

${ }^{20}{ }_{i c a n t}$ difference may exist in the techniques to be applied to achieve a trade-off between ${ }^{20}$

${ }^{21}$ energy and performance, with respect to "legacy" infrastructures. 21

22 Indeed, the impact of virtualization technologies on power consumption in public ${ }^{22}$

${ }^{23}$ telecommunication networks (PTNs) is still unclear. There is a general belief that $\mathrm{Net}^{23}$

${ }^{24}$ work Functions Virtualization (NFV) should result in reduced eneroy consumption, owing ${ }^{24}$

${ }^{25}$ to consolidation of resources and increased flexibility in turning unused hardware $(\mathrm{HW})$ on $^{25}$

26 and off as needed. However: it is also true that "the massive introduction of general-purpose

${ }^{27} \mathrm{HW}$ enabled by NFV would tend to increase power requests with respect to specialized ${ }^{27}$

${ }^{28} \mathrm{HW}$ solutions" [28]. Therefore, there is a need to operate power-aware management and ${ }^{28}$

${ }^{29}$ control mechanisms in these environments. At the same time, it is necessary to limit the ${ }^{29}$

${ }^{30}$ complexity of these mechanisms and the level of human intervention therein, to keep Op- ${ }^{30}$

31 erational Expenditures (OPEX) within reasonable limits. One approach to understanding ${ }^{31}$

${ }^{32}$ the impact consists of comparative analyses of implementations of infrastructure, with and ${ }^{32}$

${ }^{33}$ without virtualization. This approach is taken in [31], where the evolved packet core (EPC) ${ }^{33}$ 
${ }^{1}$ is studied. This work shows that the virtualized implementation is indeed less energy effi- ${ }^{1}$

${ }^{2}$ cient. Unfortunately, the scope of virtualization and containerization within the converged ${ }^{2}$

${ }^{3}$ wireless and wireline infrastructure is very broad and consideration of a single "use-case" ${ }^{3}$

${ }^{4}[32]$ cannot be generalized to an overall statement. We therefore note that the scope for our ${ }^{4}$

${ }^{5}$ survey needs an operational context and suggest one next. 5

6

${ }^{7} 1.4$ The scope of this survey ${ }^{7}$

${ }^{8} 1.4 .1$ Operational context 8

${ }^{9}$ We suggest that the "telco cloud" is our context. "Telco cloud" is an evolving notion that ${ }^{9}$

${ }^{10}$ evokes a number of common terms in attempts to describe it. Virtualization, software- ${ }^{10}$

${ }^{11}$ defined networking (SDN), automation and orchestration are four such terms. Other promi- ${ }^{11}$

${ }^{12}$ nent terms are edge computing, containerization, microservices and resilient infrastructure ${ }^{12}$

${ }^{13}$ [33]. To avoid reducing the telco cloud to a melting pot of terms requires three key obser- ${ }^{13}$

${ }^{14}$ vations that organize them into a coherent image. 14

15 The telco cloud is, fundamentally, a hybrid cloud: 15

(a) self-sourced virtualization and containerization and 16

(b) out-sourced (public cloud) containerization.

17

The complementary collaboration of the PTN operator's (PTNO) network, compute ${ }^{18}$ and storage infrastructure, with that of global providers of infrastructure and appli- ${ }^{19}$ cations, is manifested well in [34]. A distributed cloud infrastructure operates at net- ${ }^{20}$ work (transport and interconnect) junctions. It includes (cloud) infrastructure owned ${ }^{21}$ and operated by the PTNO, by public cloud providers, and by enterprises which con- ${ }^{22}$ sume their joint service.

2 Secondly, the telco cloud serves both internal and external clients [33].

(a) Internal use can suggestively be referred to as the IT Cloud [35]. This consists ${ }^{25}$ of applications specific to PTNOs: operational support systems (OSS) and busi- ${ }^{26}$ ness support systems (BSS), as well as more general applications, like customer ${ }^{27}$ relationship management (CRM).

(b) External uses are growing organically on the basis of use cases seeded by ETSI $^{29}$ [32] and the 5GPPP [36].

3 The service-based architecture (SBA) of the $5 G$ Core is a good fit with cloud- $^{31}$ native computing. Containerization is distinctively central to cloud-native comput- ${ }^{32}$ ing [37][38]. The Cloud Native Computing Foundation explicitly identifies contain- ${ }^{33}$ 
${ }_{13}^{1.4 .2}$ Model scope ers as components of the approach to the concept of cloud native computing [39]. ${ }^{1}$ There is a clear drive towards use of containers in lieu of virtual machines as the operating environment for network functions [37], and the 5G Core's SBA provides ${ }^{3}$ a clear scope for employing containers.

The VEs in context may be deployed in:

datacenters: the real estate referred to here consists of points of presence (PoPs) as metro-core PoPs at the near edge;

2 softwarized and virtualized networks: here, we refer to points of presence such as central offices (COs) and sites even deeper into the edge such as remote radio head (RRH) sites and roadside cabinets.

14We survey predictive energy and power models, as well as measurements that facilitate14 ${ }_{15}$ qualitative and/or quantitative prediction, of consumption by individual VEs relevant to the15 16telco cloud. A simple interpretation of the rationale that drove our selection is that we have16 17sought works that measure real-time power consumption by VEs and/or model real-time 17 18power consumption by VEs. The object of measurements and modelling is strictly the VE.18 19 Nonetheless, the devil is in the details and so the details of this simple rationale must ${ }^{19}$ ${ }^{20}$ be worked out. One important, finer point regards the VEs themselves. There are software ${ }^{2 \theta}$ ${ }^{21}$ technologies, which we shall elaborate upon in later sections, which are functionally criti $^{21}$ ${ }^{22} \boldsymbol{c a l}$ to VEs. Works that measure, and/or model, such technologies' power consumption are ${ }^{22}$ ${ }^{23}$ in scope. Justification of this claim on scope is not hard. Since power consumption is a ${ }^{23}$ ${ }^{24}$ scalar quantity, reduction of power consumption of a component of a VE translates into ${ }^{24}$ ${ }^{25}$ reduction of power consumption by the VE. In fairness, the translation is not direct $(1: 1){ }^{25}$ ${ }^{26} \mathrm{~A}$ generalization of Amdahl's law comes to mind: that improvement in a component, mea- ${ }^{26}$ ${ }^{27}$ surable by some metric, is attenuated by the ratio of that component's use (measureable by ${ }^{27}$ ${ }^{28}$ that metric) to the system's (the VE's) use (measurable by the same metric). However, we ${ }^{28}$ ${ }^{29}$ can safely summarize the finer point we alluded to at the start of this paragraph, as follows. ${ }^{29}$ ${ }^{30}$ Research that studies measurement and modelling of power consumption by a component ${ }^{30}$

${ }^{31} \mathrm{of} \mathrm{a} \mathrm{VE} \mathrm{is} \mathrm{in} \mathrm{scope.} \mathrm{We} \mathrm{clarify} \mathrm{this} \mathrm{by} \mathrm{example.} \mathrm{For} \mathrm{instance,} \mathrm{we} \mathrm{would} 31$

32 Include the Data Plane Development Kit (DPDK) [40], as it serves the critical func- ${ }^{32}$ tion of networking (VEs that serve as virtual network functions (VNFs)); 
12 Include a comparative study that measures power consumption by a VE using two ${ }^{1}$ 2 different implementations of input/output virtualization technology, say: SR-IOV ${ }^{2}$ (single-root IO virtualization) and paravirtualization;

$4 \quad 3$ Exclude a comparative study that measures power consumption by various network ${ }^{4}$

5 adapter (or network interface card (NIC)) architectures, unless it reveals the impact ${ }^{5}$

6 of these architectures on VEs' power consumption. 6

Further detail emerges from the "real-time" requirement. This term is a reflection of the

${ }^{8}$ need for application agnosticism. Power meters that follow from such measurements and ${ }^{8}$

9 models may be used regardless of whether instantaneous or statistical readings of power ${ }^{9}$

${ }^{10}$ are required. Studies (on measurements and models) meet this requirement by satisfying ${ }^{10}$

11 the following criteria.

12

13

1 Predictors

(a) must be of fine temporal granularity;

14

(b) must be updated with the regularity of the temporal granularity;

(c) must enable prediction of power consumption at the same temporal granularity.

2 Workload: Only resource-specific constraints are considered (e.g. processor-intensive). In particular, models must not constrain the stochasticity of the workload.

Our last detail regards workload. We observe, in section 4.4.1, that the universal power ${ }_{20}$ model is a fallacy and the principal reason is that interaction between workload and ar${ }_{21}$ chitecture cannot be pinned down indefinitely. This does not mean that modelling is $a_{21}$ ${ }_{22}$ fruitless endeavour. It simply means that validity constraints must be placed on the model ${ }_{22}$ ${ }_{23}$ in terms of workload and architecture. Therefore, we do not exclude modelling and mea- ${ }_{23}$ ${ }_{24}$ surement because of its workload-scope or architectural scope. We do, however, observe ${ }_{24}$ ${ }_{25}$ that such models are pitfalls for those who apply them without knowledge of such limits ${ }_{25}$ ${ }_{26}$ (section 4.3.3).

27 We now proceed to present some recent, related surveys, highlighting their methodology 27 ${ }_{28}$ and analytical approach. Against this background, we summarize the novelty of this survey ${ }_{28}$

${ }^{30} 1.5$ Related surveys $\quad 30$

${ }^{31}$ In recent years, a lot of research work has been done for modeling power for servers in data ${ }^{31}$

${ }^{32}$ centers and cloud environment in general. Several surveys in this regard cover different ${ }^{32}$

33 aspects of modeling and energy efficiency approaches for servers and virtual entities. 33 aspects of modeling and energy efficiency approaches for servers and virtual entities. 
1 An analysis of power models from micro- to macro-level is presented in [41]. This survey ${ }^{1}$

${ }^{2}$ covers different aspects and levels of both hardware- and software-centric modeling tech- ${ }^{2}$ ${ }^{3}$ niques. Researchers studied the models based on the computing resources (CPU, mem- ${ }^{3}$ ${ }^{4}$ ory, storage and I/O), system architecture (such as single or multiple cores), availability ${ }^{4}$ ${ }^{5}$ of Graphics Processing Units (GPUs), system/network components, operating system and ${ }^{5}$ ${ }^{6}$ virtualization environments. They categorize the existing models at different layers mov- ${ }^{6}$ ${ }^{7}$ ing from architecture level modeling to the level of power models for whole data centers. ${ }^{7}$ ${ }^{8}$ However, the survey did not focus greatly on power modeling techniques that consider the ${ }^{8}$ ${ }^{9}$ effect of different virtual entities.

10 According to the categorization in [41], power models depend on different organizational ${ }^{10}$

${ }^{11}$ context including power consumed by system components, running applications, and/or ${ }^{11}$

${ }^{12}$ execution strategy of processes. These metrics, however, can derive additive component- ${ }^{12}$

${ }^{13}$ based, regression-based or machine-learning-based power models. Additive models usu- ${ }^{13}$

${ }^{14}$ ally present an aggregated view of server power consumption, which could be based on ${ }^{14}$

${ }^{15}$ different resources (such as cpu, ram, I/O, disk), or disaggregate static and dynamic power ${ }^{15}$

${ }^{16}$ consumption of the server. Regression based models are mostly based on the relation of ${ }^{16}$

${ }^{17}$ power to the dynamic evolution of some measured system parameters. Power modeling ${ }^{17}$

${ }^{18}$ using machine learning techniques is an advanced research area which can be further clas- ${ }^{18}$

${ }^{19}$ sified as supervised, unsupervised, reinforcement and evolutionary learning. Furthermore, ${ }^{19}$

${ }^{20}$ at a much higher level, such as that of data center environments as a whole, power models ${ }^{20}$

${ }^{21}$ for group of servers, datacenter networks, conditioning and cooling systems were studied. ${ }^{21}$

${ }^{22}$ The survey also analyzed the modeling methods at operating system (OS) and virtualiza- ${ }^{22}$

${ }^{23}$ tion level, and for data-intensive, communication-intensive, and general applications. $\mathrm{At}^{23}$

${ }^{24}$ the end, the researchers compare the power models against their complexity, effectiveness, ${ }^{24}$

${ }^{25}$ application and use-cases.

${ }^{26}$ The survey carried out in [42], analyzes the power models based on their modeling ap- ${ }^{26}$ ${ }^{27}$ proaches. According to it, power modeling methods can be divided into two main themes; ${ }^{27}$

${ }^{28}$ analytical models and formula-learned models. In the former, parameters affecting the sys- ${ }^{28}$

${ }^{29}$ tem power are known to the researcher; however, the weight for each parameter needs to ${ }^{29}$

${ }^{30}$ be determined. On the contrary, in high-level formula-learned or machine learning based ${ }^{30}$

${ }^{31}$ modeling, no prior knowledge of the system is required and the model is developed from ${ }^{31}$

${ }^{32}$ scratch using the provided data set. The survey further describes the commonly used ma- ${ }^{32}$

${ }^{33}$ chine learning techniques, and later focuses on the use and effectiveness of neural networks ${ }^{33}$ 
${ }^{1}$ in power modeling. According to this survey, the approach to model power for any server ${ }^{1}$

${ }^{2}$ may vary in several aspects. These different characteristics are summarized as i) degree of ${ }^{2}$ ${ }^{3}$ autonomy, i.e. to which level the power model is dependent on external hardware, ii) level $^{3}$ ${ }^{4}$ of granularity, which describes the depth of logical (core, thread, etc.) or physical (de- ${ }^{4}$

${ }^{5}$ vice specific, system level, etc.) level to which the model can precisely estimate the power, ${ }^{5}$ ${ }^{6}$ iii) methodology, describing the selection of method, which could be simulation, analytical ${ }^{6}$

${ }^{7}$ modeling or data-training, iv) simplicity, which can be assessed by the number of variables, ${ }^{7}$

${ }^{8}$ method selection and models' computing overhead, v) portability, as most of the models ${ }^{8}$

${ }^{9}$ developed are effective for some specific architecture, workload, or environment, and their ${ }^{9}$

${ }^{10}$ generalization is still a question, vi) accuracy, which defines the measurement accuracy or ${ }^{10}$

${ }^{11}$ estimation precision of power for any model, and vii) power meter (as most studies use ${ }^{11}$

${ }^{12}$ external power meters as a ground truth for their modeling, their accuracy is also a major ${ }^{12}$

${ }^{13}$ concern for developing a more efficient model). 13

14 The survey in [43] adopts a slightly different approach of analysis and evaluates the ${ }^{14}$

${ }^{15}$ selected existing power modeling techniques in a unified environment. The comparative ${ }^{15}$

${ }^{16}$ analysis has been performed for 24 different software power models and measurement ${ }^{16}$

${ }^{17}$ methods with 9 different benchmarks under a single experimental environment. It evalu- ${ }^{17}$

${ }^{18}$ ates the existing software power measurement techniques and models for different applica- ${ }^{18}$

${ }^{19}$ tions, benchmarks, system configuration, server architecture and for their estimation errors. ${ }^{19}$

${ }^{20}$ According to [43], most of the software-based power models use system performance met- ${ }^{20}$

${ }^{21}$ rics provided by the operating system, or performance monitoring counters provided by ${ }^{21}$

${ }^{22}$ hardware sub-systems of the server. Software power models considered in this study are ${ }^{22}$

${ }^{23}$ categorized in three types as single variable CPU-based, multi-variable CPU-based, and ${ }^{23}$

${ }^{24}$ single-variable throughput-based. The result of this unified experimental setup shows that ${ }^{24}$

${ }^{25}$ power models based on support vector machine (SVM) and interpolation techniques shows ${ }^{25}$

${ }^{26}$ the least error for different resource-intensive applications, whereas lasso regression with ${ }^{26}$

${ }^{27} 30$ variables was found to be the worst power model with the highest error. Also the mod- ${ }^{27}$

${ }^{28}$ eling techniques are mainly divided into two categories; linear and non-linear, where each ${ }^{28}$

${ }^{29}$ category is further classified based on its derivation from mathematical modeling or ma- ${ }^{29}$

${ }^{30}$ chine learning techniques.

31 Another survey [44], with a more limited scope, focuses on the power modeling of servers ${ }^{31}$

${ }^{32}$ in the cloud while considering complexity of virtualization environment, heterogeneity and ${ }^{32}$

${ }^{33}$ workload complexity. It presents the usability, applicability and limitations of the studied ${ }^{33}$ 
${ }^{1}$ approaches, and also presents the analysis of traditional existing and emerging modeling ${ }^{1}$

${ }^{2}$ techniques. It reviews the existing power modeling methods at three stages: data acquisi- ${ }^{2}$

${ }^{3}$ tion methods, power consumption models and power modeling methods for servers, $\mathrm{VMs}^{3}$

${ }^{4}$ and containers. According to this study, the collection of data for power models could be ${ }^{4}$

${ }^{5}$ based on: a) instruments such as external power meters, b) dedicated acquisition systems ${ }^{5}$

${ }^{6}$ which are generally customized products by developers for a specific hardware, c) simu- ${ }^{6}$

${ }^{7}$ lation based, and d) software monitoring tools. The latter constitute a widely used method ${ }^{7}$

${ }^{8}$ that is based on system indicators and sensors. For the second stage, i.e. the power mod- ${ }^{8}$

${ }^{9}$ eling, either hardware-centric, virtualization-centric or application centric power modeling ${ }^{9}$

${ }^{10}$ schemes can be used. Each type has its own drawback and usability; hence, the domain is ${ }^{10}$

${ }^{11}$ still an open research area for further generalization and measurement precision. The se- ${ }^{11}$

${ }^{12}$ lection of method which is considered as third step of the modeling in [44] is characterized ${ }^{12}$

${ }^{13}$ as power modeling based on: a) empirical parameterization, b) function regression, c) ma- ${ }^{13}$

${ }^{14}$ chine learning, and d) evolutionary algorithms. The survey, after analyzing different power ${ }^{14}$

${ }^{15}$ models, concluded that functional regression and machine learning methods yield high ${ }^{15}$

${ }^{16}$ accuracy when provided with large enough data sets and clear power behavior of servers. ${ }^{16}$

${ }^{17}$ This survey is more similar to the present one; however, we distinguish further by providing ${ }^{17}$

${ }^{18}$ statistical analysis of existing approaches, used tools, methods and measurement metrics. ${ }^{18}$

${ }^{19}$ Also, our survey focuses on modeling of power for individual virtual entities and on the ${ }^{19}$

${ }^{20}$ effect of the virtualization environment on server and network functions power behavior. ${ }^{20}$

${ }^{21}$ In general, the surveys mentioned above present the literature of server models irrespec- ${ }^{21}$

${ }^{22}$ tively of the operational environment. One of the major trends, not only in data centers ${ }^{22}$

${ }^{23}$ but also in networking is the presence of virtual entities and the adoption of virtualiza- ${ }^{23}$

${ }^{24}$ tion environments. In this respect, this survey concentrates on power models of virtualized ${ }^{24}$

${ }^{25}$ entities, and focuses mainly on components and parameters that could affect their power ${ }^{25}$

${ }^{26}$ consumption. It further provides a statistical analysis of included research works with re- ${ }^{26}$

${ }^{27}$ spect to their data acquisition methods, measurement tools and modeling methods, to give ${ }^{27}$

${ }^{28}$ researchers a bird's-eye view of the existing literature through which one can identify the ${ }^{28}$

$\begin{array}{ll}29 & \text { approaches carried out in different studies. } \\ & 29\end{array}$

$30 \quad 30$

${ }^{31} 1.6$ Intended audience $\quad 31$

${ }^{32}$ Our intended audience is the prospective researcher, seeking to understand the dynamics ${ }^{32}$

${ }^{33}$ of research into predictive modelling, and supporting measurements, of power consump- ${ }^{33}$ 


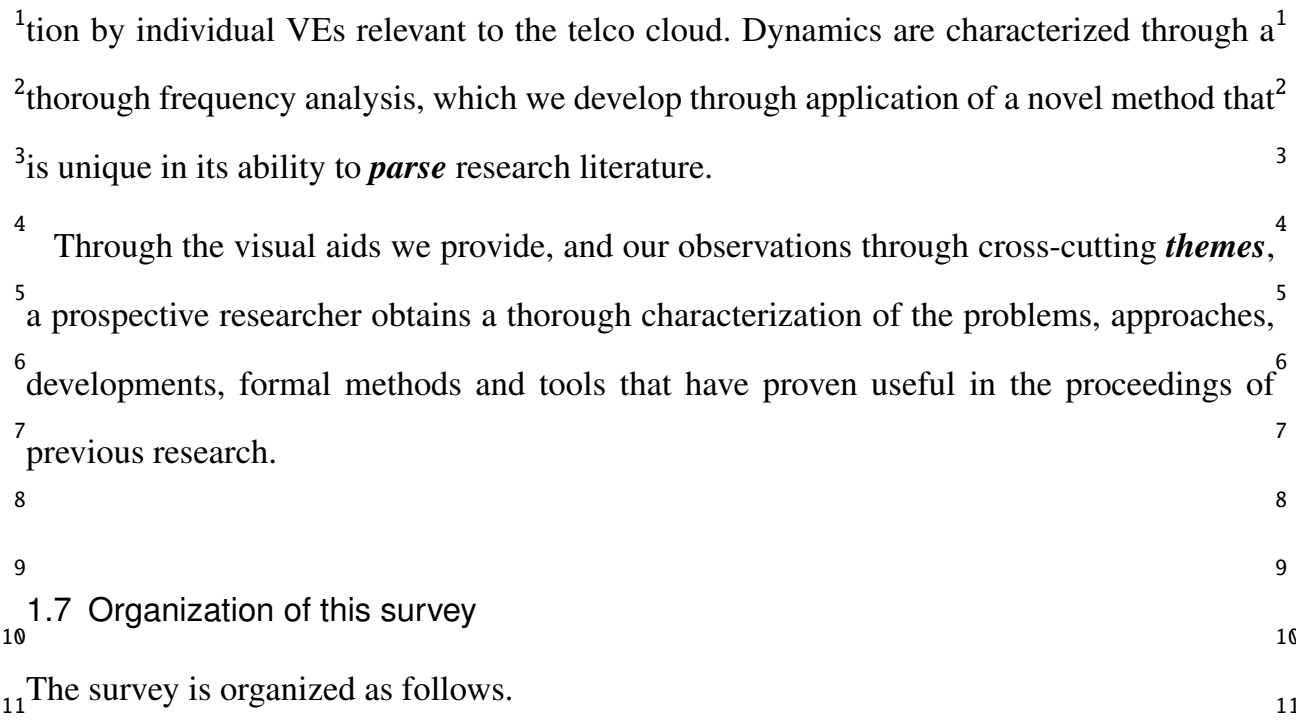

121 In [45], we describe our method: a problem-approach-development (PAD) triad,12 13 which, to the best of our knowledge, we are the first to use, to identify research $_{13}$ 14 dynamics. We have delegated description of the method to a separate paper, to re-14 15 solve the difficulty of elaborating fully on the method without distracting attention 15 from the results which we have obtained and which we document separately in this 16 review. our scope.

2 an appendix (section 6), which describes and classifies applications that depend upon ${ }^{29}$ accurate models of power consumption by virtual entities to execute their function ${ }^{30}$ effectively, and

3 another appendix (section 7), where we describe and compare tools and methods ${ }^{32}$ employed by researchers in their pursuit of developments within their field of study. ${ }^{33}$ 


\section{Research Domains (interest categories)}

${ }^{2}$ We observe a (limited) number of interests which give rise to research within our scope. ${ }^{2}$ ${ }^{3}$ These are:

1 predictive models of real-time power consumption;

5

3 .

It is important to note that interests are latent: they are not usually explicitly stated within

RUs. Researchers' interests - what motivated them to go through the pains of research - are 10 deduced through knowledge of the underlying field and comparison with other RUs from a high level. This is particularly diffic 11 sumption; researchers may have very diverse complementary disciplines (the discipline 12 which they are studying for energy consumption). There is a real risk of subjectivity in 13 parsing, although this is mitigated by group review (as we have done) and experience. No14 15 the sharper quirks of individuals' subjectivity. 16 ${ }_{17}$ We use this section to separate interest categories from the others ( $\mathrm{P}, \mathrm{A}$ and $\left.\mathrm{D}\right)$, for two ${ }_{17}$ reasons:

191 to avoid repeating essentially the same three interest categories with every RU ana- ${ }_{19}$ 20 lyzed, and

212 to avoid cluttering the remaining, more objective data with data that is always subject ${ }_{21}$ to the suspicion of subjectivity.

23 We close this introduction to the section on research domains with an important clari-23 ${ }_{24}$ fication. The research domains are not our scope: they encompass it. Our scope remains ${ }_{24}$ ${ }_{25}$ research on measurement of power consumption by VEs and models which predict their ${ }_{25}$ ${ }_{26}$ power consumption.

${ }^{29}$ Table 2 shows a list of the techniques we have detected for building power models. $\mathrm{We}^{29}$

${ }^{30}$ follow up on this table with a brief historical perspective of the development of these tech- ${ }^{30}$

${ }^{31}$ niques to meet the demands of the scope of VEs.

${ }^{32}$ Cycle-accurate simulators like SimplePower [46] and Wattch [47] extended application ${ }^{32}$

${ }^{33}$ of low-level simulation to estimation of power consumption in processors. The cost of ${ }^{33}$ 


\begin{tabular}{lllll}
\hline $\begin{array}{l}2 \\
\text { Technique }\end{array}$ & Scope & \multicolumn{2}{l}{$\begin{array}{l}\text { Sample } \\
\text { references }\end{array}$} \\
\hline
\end{tabular}

${ }_{11}$ low-level simulation is complexity, rendering this technique (i.e. simulating dynamics at ${ }_{11}$ ${ }_{12}$ register-transfer or gate level) unsuited to real-time estimation of power consumption. ${ }_{12}$ 13 System-software instrumentation modeling is referred to in [55] and [41] as the "top-13 14down" method. It is "architecture agnostic" [55] and uses system software's abstractions of 14 15system hardware to count the occurrence of specific events. Such event counters are proxies 15 16 for rates of hardware - and thus power - use. This is the intuitive basis for the use of event16 17 counters as predictors of power consumption. We point out that the terms "event counter"17 18and "performance monitoring counter" are largely used synonymously in the literature we18 19have reviewed.

20 A seminal work in correlation between energy consumption and processor hardware ${ }^{20}$ ${ }^{21}$ events investigated the latter's use in the scheduling of threads in an energy-aware man- ${ }^{21}$ ${ }^{22}$ ner [51]. Later, it was found [56] that the counter value for the number of instructions ${ }^{22}$ ${ }^{23}$ fetched can be used to estimate the system's power consumption. These two works rep- ${ }^{23}$ ${ }^{24}$ resent a departure from system-software instrumentation modeling (top-down) as they are ${ }^{24}$ ${ }^{25}$ predicated upon a knowledge of functional units within the computer system, particularly ${ }^{25}$

${ }^{26}$ at the microarchitectural level. 26 27 This technique was developed into what has come to be known as functional level power ${ }^{27}$ ${ }^{28}$ analysis. A significant advance in prediction of power consumption took place through the ${ }^{28}$ ${ }^{29}$ use of abstraction of processor operation into a set of functional blocks. Each such block ${ }^{29}$ ${ }^{30}$ implements a function recognizable at the semantic level of the microarchitecture, e. in $^{30}$ ${ }^{31}$ struction fetch and data fetch. This analytical approach was first referred to as functional ${ }^{31}$ ${ }^{32}$ level power analysis (FLPA) in [53]. The major components of this approach are still rele- ${ }^{32}$ ${ }^{33}$ vant to most of the modeling work which we have surveyed, i.e.: 
$1 \quad 1$ knowledge of microarchitecture

22 knowledge of interaction of program code and architecture 2

$3 \quad 3$ workloads that isolate specific resources.

4 Another name given to this technique $[41,55]$ is "bottom-up", in recognition that a power

121 functional level power analysis [52, 53] and 12

132 power consumption - hardware event correlation [51], respectively. 13

${ }^{14}$ We observe that this domain's practitioners are biased strongly towards these two bases as ${ }^{14}$

${ }^{15}$ their analytical premises.

${ }_{18}^{2.2}$ Research domain \# 2: Power/energy - efficient computer and network system 18 integration

${ }^{20}$ Several works are concerned with measurement of power consumption in virtualized en-20 ${ }^{21}$ vironments but stop well short of striving for predictive models [57-63]. These works ${ }^{21}$ ${ }^{22}$ seem to stem from an interest in obtaining causal relationships between, on the one hand, ${ }^{22}$ ${ }^{23}$ some aspect of implementation of computer and network systems, and on the other hand, ${ }^{23}$ ${ }^{24}$ power/energy consumption/efficiency. A wide variety of aspects can be found: non-uniform ${ }^{24}$ ${ }^{25}$ memory access (NUMA) and simultaneous multi-threading (SMT) [57], scaling execution ${ }^{25}$ ${ }^{26}$ over multiple processor cores [58], virtualization technology [59], virtualization genre [60], ${ }^{26}$ ${ }^{27}$ data plane [62] and network functions [63]. 27 28 Research of this kind might also stem from a perceived opportunity to break new re- ${ }^{28}$ 29 search ground and harvest the low-hanging fruit of an unexplored research space. The term ${ }^{29}$ ${ }^{30}$ "profiling", as used in [58], is indeed an apt description of research activities rooted in ${ }^{30}$ ${ }^{31}$ this common interest. However, even if this were the case, IT discipline skills exhibited in ${ }^{31}$ ${ }^{32}$ such research is suggestive of an interest in computer and network system integration. We ${ }^{32}$ 33 propose this as the common root. 
${ }^{1} 2.3$ Research domain \# 3: Power-efficient, high-performance packet I/O frameworks ${ }^{1}$

${ }^{2}$ General-purpose network I/O has been implemented in stacks of software modules operat- ${ }^{2}$

${ }^{3}$ ing in the kernel of general-purpose operating systems. To date, these network I/O kernel ${ }^{3}$

${ }^{4}$ stacks are incapable of transmitting and receiving at $10 \mathrm{~Gb} / \mathrm{s}$ line rates [64]. Higher per- ${ }^{4}$

${ }^{5}$ formance packet I/O frameworks have been developed, e.g. SR-IOV, DDIO (Data Direct ${ }^{5}$

${ }^{6} \mathrm{I} / \mathrm{O}$ ), DPDK and, more recently, AF_XDP (Address Family eXpress Data Path). Exploita- ${ }^{6}$

${ }^{7}$ tion of SR-IOV's VFs for high performance is hardware-dependent; this dependency ren- ${ }^{7}$

${ }^{8}$ ders SR-IOV problematic to the vision of cloud-native computing: running anywhere [65]

${ }^{9} @ 52: 01^{[1]}$. This hardware-dependence contrasts with DPDK and AF_XDP, which are less ${ }^{9}$

${ }^{10}$ restrictive in hardware specification. DPDK's cost might be power consumption [62-64] ${ }^{10}$

${ }^{11}$ (depending on its usage, as discussed in section 4.3.2)and AF_XDP's power-performance ${ }^{11}$

${ }^{12}$ tradeoff is still comparatively unknown. 12

13

High-performance packet I/O's centrality to the success of NFV seems likely to turn it

${ }^{14}$ into a rich source for future research. In addition, the pressing need to control operational ${ }^{14}$

15 expenditure should maintain interest in power efficiency. The conjunction of these two

${ }^{16}$ interests is the noteworthy research domain of power-efficient, high-performance packet ${ }^{16}$

17.17

I/O frameworks.

$18 \quad 18$

$19 \quad 19$

3 Survey results: a Digest of Challenges, Approaches and 20 Developments

21

${ }_{22} 3.1$ A taxonomy of the problem space

${ }_{23} \mathrm{As}$ our parsing efforts proceeded, we observed that the scope of this survey is relatively ${ }_{23}$ ${ }_{24}$ narrow and the problems in our set are not fully independent of one another. Rather, the ${ }_{24}$ ${ }_{25}$ problems diverge from one another only as aspects (we could also say that they are deriva-25 ${ }_{26}$ tives) of the core challenge of modeling the power consumption of virtualized entities. 26 ${ }_{27}$ Each RU is rooted in this core challenge, but the derivative problems (our P-categories and ${ }_{27}$ 28 their members) addressed differ from one RU to another. Fig. 1 is an illustration of a sim-28 29ple organization of the challenges which have been tackled in the literature and shows their 29 ${ }_{30}$ frequency of presence in RUs. The organization gives prominence to how challenges have ${ }_{30}$ 31been perceived: $\quad 31$

${ }^{32}{ }^{11}$ We use the @ symbol since a video is being cited and the content cited here may be found ${ }^{32}$

${ }^{33}$ by seeking the time indicated. 
11 one group regards the concern with obtaining an understanding of the dependency ${ }^{1}$

2 of power consumption on some genre of artifacts. Categories P1,2 and P9 - 11 are in ${ }^{2}$

3 this group.

42 the other regards the concern with how to predict power consumption. Categories $\mathrm{P} 3^{4}$

$5-8$ and P12 are in this group.

${ }^{6}$ Category P13 regards a very recent development of the problem space and works classified ${ }^{6}$

${ }^{7}$ here present both dependency and modeling concerns.

${ }^{8}$ We now proceed to describe the categories in more detail. Each description is preceded ${ }^{8}$

${ }^{9}$ by a list of references to works that tackle a challenge in the problem category.

10

${ }^{11}$ 3.1.1 P1: Host system hardware architecture perspective: dependency of VE power 11 12 consumption on host system architecture 12

${ }^{13}[57,58,63,66]$. Problem category P1 addresses the impact of specific architectural at- ${ }^{13}$

${ }^{14}$ tributes of the host system on power consumption of VEs. On the one hand are the major ${ }^{14}$

${ }^{15}$ attributes of architecture and system-level design. On the other hand are the changes in ${ }^{15}$

${ }^{16}$ power consumption (the behavior) as major features are adjusted, inserted or removed. ${ }^{16}$

${ }^{17}$ Insertions and removals are coarse configurative actions such as enabling or disabling; ad- ${ }^{17}$

${ }^{18}$ justments consist of progressive modifications such as adding increments of a resource. ${ }^{18}$

${ }^{19}$ Examples of features which have been tackled include multiple processor cores, processor ${ }^{19}$

${ }^{20}$ frequency scaling, NUMA and hardware threads (e.g. Intel Hyper-Threading).

21 For network functions, the importance of knowledge about the power efficiency of ${ }^{21}$

${ }^{22}$ NUMA and multiple-core architectures has the added relevance of these architectures ${ }^{22}$

${ }^{23}$ relationship to determinism [65] @ 19:00, @ 25:25. We dwell further on the underlying ${ }^{23}$

${ }^{24}$ premise of hard partitioning in our consideration of the impact of the high-performance ${ }^{24}$

${ }^{25}$ data plane on power efficiency (section 4.3.2).

26 Research that investigates the dependency of power consumption on architecture is ex- ${ }^{26}$

${ }^{27}$ ploratory, charting work. It attempts to provide a framework for detailed modeling through ${ }^{27}$

${ }^{28}$ the discovery of broad relationships. Problems in this category arise with developments ${ }^{28}$

${ }^{29}$ in architecture and system-level design. For example, while [58] is a comparatively old ${ }^{29}$

${ }^{30}$ work that tackles architecture, [57] is newer and finds scope for research in system soft- ${ }^{30}$

31 ware's exploitation of NUMA. One recent, highly significant scope is that of the use of

32 domain-specific architectures (DSAs). Researchers are exploring specialized hardware in ${ }^{32}$

${ }^{33}$ the quest for improvement of the energy-performance-cost ratio, and will investigate en- ${ }^{33}$ 
${ }^{1}$ ergy efficiency in the process of their research. As domain-specific cores are mixed with ${ }^{1}$

${ }^{2}$ general-purpose cores, many architectures will be investigated from each of the three pin- ${ }^{2}$

${ }^{3}$ nings: energy consumption, performance and cost.

4 A particularly relevant set of DSAs regards real-time packet processing by computer ${ }^{4}$

${ }^{5}$ systems hosting NFs at intermediate nodes (INs) at the network edge. Concern lies with ${ }^{5}$

${ }^{6}$ expediting the common tasks, such as sending/receiving packets and processing headers. ${ }^{6}$

${ }^{7}$ SR-IOV is a good example (see, for example [67], and its inclusion in [63]), but software- ${ }^{7}$

${ }^{8}$ only solutions, such as poll-mode drivers, may also help to cut through the many middle- ${ }^{8}$

${ }^{9}$ men characteristic of general-purpose operating systems [62, 63]. Introduced to serve the ${ }^{9}$

${ }^{10}$ perspective of performance, it is now necessary to understand their impact on power effi- ${ }^{10}$

${ }^{11}$ ciency. Therefrom, it is necessary to understand how to control their power consumption. ${ }^{11}$

12 We suggest that "profiling", the term chosen in [58] and [68], is a helpful descriptor of ${ }^{12}$

${ }^{13}$ this kind of research. Just as a profile produces an external boundary within which to fill ${ }^{13}$

${ }^{14}$ detail, so does this kind of research provide a framework through which modeling work is ${ }^{14}$

${ }^{15}$ facilitated and within which modeling work provides details of power consumption rela- ${ }^{15}$

${ }^{16}$ tionships. 16

$17 \quad 17$

${ }^{18}$ 3.1.2 P2: System perspective: impact of alternative virtualization genres and $\quad 18$

19 virtualization platforms on VE power consumption 19

${ }^{20}[59,60]$. Here, a behavior that consumes power is investigated across different implementa- ${ }^{20}$

${ }^{21}$ tions of a system concept. We have observed investigation of two different system concepts: ${ }^{21}$

${ }^{22}$ (a) virtualization genres and (b) virtualization platforms. There are three members of the ${ }^{22}$

${ }^{23}$ virtualization genre group: containers, para-virtualization and hardware-assisted virtualiza- ${ }^{23}$

${ }^{24}$ tion. In the virtualization platform group, examples include Xen, Hyper-V, Kernel Virtual ${ }^{24}$

${ }^{25}$ Machine (KVM [69]), Docker and Linux Containers (LXC). 25

26 Research questions (problems) typical of category P2 seek to control scope of experi- ${ }^{26}$

${ }^{27}$ mentation through exercise of specific resources, e.g. per-host networking using emulated ${ }^{27}$

${ }^{28}$ switches (software switches) [59, 60], processor-bound and memory-intensive processing ${ }^{28}$ ${ }^{29}[59]$.

30 We consider genres and platforms as sub-categories of the same overarching problem ${ }^{30}$

${ }^{31}$ category. Namely, this is system-level exploration that attempts to establish generalizations ${ }^{31}$

${ }^{32}$ about an uncharted space. Like problems in category P1, new problems in this category ${ }^{32}$

${ }^{33}$ arise with fresh alternative virtualization genres and platforms. However, here the scope ${ }^{33}$ 
${ }^{1}$ of investigation is broader than with works classified under P1. Unlike P1, where specific ${ }^{1}$

${ }^{2}$ architectural aspects (e.g. NUMA, hardware threads) are explored, the perspective taken ${ }^{2}$

${ }^{3}$ here is concern with the impact of a choice of implementation of a system. ${ }^{3}$

4

53.1.3 P3: Estimation of power consumption of (virtualization-) host system 5

${ }^{6}[70-82]$. Measurement of a single server's power consumption through the use of an exter- ${ }^{6}$

${ }^{7}$ nal power meter is a trivial task. However, at the scale of cloud datacenters, it is a logistical ${ }^{7}$

${ }^{8}$ burden. In addition, travel to the datacenter's site may be burdensome. Furthermore, ser- $^{8}$

${ }^{9}$ vice availability would be reduced by the process of attaching a physical power meter to ${ }^{9}$

${ }^{10}$ the hardware in the virtualization platform, e.g. between the server's power inlet and the ${ }^{10}$

${ }^{11}$ outlet in the racking cabinet's power distribution unit (PDU) (naturally, availability would ${ }^{11}$

${ }^{12}$ only be affected in cases that do not integrate (management and) measurement facilities ${ }^{12}$

${ }^{13}$ within the PDU). 13

14 The alternative is deployment of software power meters. In the scope of this survey, the ${ }^{14}$

${ }^{15}$ cases we consider are meters that attempt to predict host power consumption on the basis ${ }^{15}$

${ }^{16}$ of activity in the $\boldsymbol{V E}$. This challenge is tackled, for example, in [52, 71-73, 75, 80]. These ${ }^{16}$

${ }^{17}$ works then proceed to tackle the problem of attribution of system power to the guest VEs. ${ }^{17}$

${ }^{18}$ Indeed, inclusion within scope of both challenges (modeling power consumption of VEs ${ }^{18}$

${ }^{19}$ and that of the host system power) seems to significantly enhance the usefulness of such ${ }^{19}$

${ }^{20}$ research, with relatively less effort. 20

21 Host power consumption may be predicted in terms of VE resource utilization, or in ${ }^{21}$

${ }^{22}$ terms of a simple characterization of the VEs' workload. Use of simple workload char- ${ }^{22}$

${ }^{23}$ acterization as predictor requires knowledge of workload parameters like number of pro- $^{23}$

${ }^{24}$ cesses, number of threads, web interactions per second and network interface utilization. ${ }^{24}$

${ }^{25}$ Enokido's and Takizawa's work [80, 81, 83] is noteworthy in its consistency in modeling in ${ }^{25}$

${ }^{26}$ these terms but other variants of this approach have been found, as (a) web interactions per ${ }^{26}$

${ }^{27}$ second [78] and (b) number of running VMs [79] of processor- and/or network-intensive ${ }^{27}$

${ }^{28}{ }^{2}$ orkloads. 28 workloads.

${ }^{29}$ To contrast: works like [70, 84-88] are not included within this category - notwithstand- ${ }^{29}$

${ }^{30}$ ing their development of models for prediction of host system power consumption. In these ${ }^{30}$

${ }^{31}$ works, host system models were developed as part of the scope of the challenge of mod- ${ }^{31}$

${ }^{32}$ eling virtualized entities. Therefrom, the challenge of system power attribution (problem ${ }^{32}$

33 category P8) was tackled to proceed to guests' power models. 33

category P8) was tackled to proceed to guests' power models. 


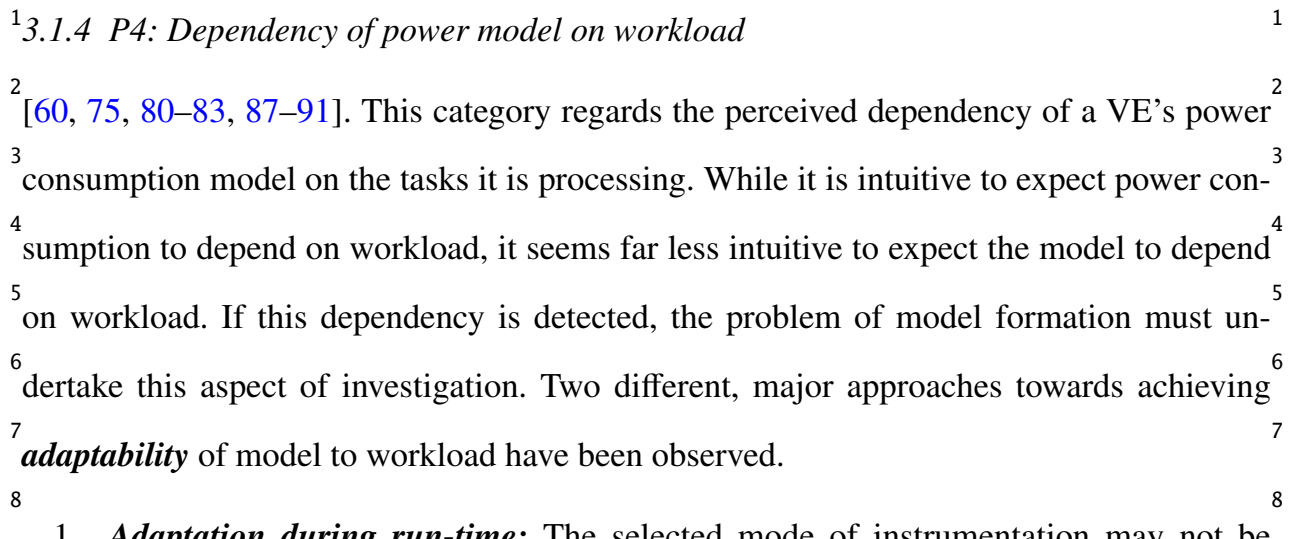
${ }_{17}$ specific. The term "homogeneous" is encountered in literature to refer to the case where ${ }_{17}$ ${ }_{18}$ host system deployments within scope are subjected to a single workload. The term seems ${ }_{18}$ ${ }_{19}$ to originate in warehouse scale computing (WSC). Conclusions drawn from this kind of ${ }_{19}$ ${ }_{20}{ }^{\text {Workload have drawn criticism as the results, while significant by virtue of the mass of }}{ }_{20}$ ${ }_{21}$ WSC, are not generalizable. The term "specific" identifies a single application; for $\mathrm{ex}^{-}{ }_{21}$ ${ }_{22}$ ample, a member of the Standard Performance Evaluation Corporation (SPEC) CPU2006 ${ }_{22}$ ${ }_{23}$ suite [92]. This term is used to indicate that models tested under such a workload are ${ }_{23}$ ${ }_{24}$ application-dependent and are valid only within a limited range of the this dimension of ${ }_{24}$ ${ }_{25}$ variability (“workload", see section 4.1.1).

26

27 3.1.5 P5: Dependency of VE's power consumption and model on VE's resource 27 28 configuration (heterogeneity)

${ }^{29}[75,85,90]$ This category regards the perceived dependency of a VE's power consumption ${ }^{29}$

30 ${ }^{30}$ and/or the dependency of its power consumption model on (a) the physical host's resource ${ }^{31}$ configuration and (b) the individual VE's resource allocation. Research here is concerned ${ }^{31}$ ${ }^{32}$ with a very practical problem: the impact on power consumption of the differences between ${ }^{32}$

${ }^{33}$ hosting machines/containers (case (a)) and the differences between virtual machines (case ${ }^{33}$ 
$\left.{ }^{1}(b)\right)$. We have observed that occurrences of research that undertake the challenge tackle it ${ }^{1}$

${ }^{2}$ as an adjoint to another focus, not as the research's primary objective. 2

3 Physical host configuration Host machines in a cloud datacenter may be expected to ${ }^{3}$

4 come in a limited variety of models, principally related to resource capacities such as ${ }^{4}$

5 the number of processor packages per server, cores per processor package, amount ${ }^{5}$

6 of RAM per server, spread in storage device sizes, etc. Processor power consumption ${ }^{6}$

7 is notably variable, even within a single family of processors. Indeed, specialization ${ }^{7}$

8 in optimized power consumption within a family is part of the study carried out in ${ }^{8}$

9 [31] within the context of an edge cluster for use in NFV. As a VE migrates from one ${ }^{9}$

10 processor within a family to a processor of different specialization, the model will ${ }^{10}$

11 differ 11

122 Individual VE's resource allocation The power consumed by a VE varies with al ${ }^{12}$

13 location of resources to (i.e. in use by) a VE, which can be dynamically varied, ${ }^{13}$

14 the number of virtual cores assigned to a VE being a notable example; see, e.g. ${ }^{14}$

$15[70,74,84-86]$. Moreover, VEs are commonly offered in sizes, e.g. small, medium ${ }^{15}$

16 and large, where allocation varies within all the major resource categories, demand- ${ }^{16}$

$17 \quad$ ing prediction of power consumption matched to the size of the purchased VE. 17

${ }_{19}^{3}$ 3.1.6 P6: Impact of temperature and/or frequency on models that predict VEs' power 19

20 consumption

${ }_{21}[73,93,94]$. This category regards the challenge of inclusion of processor package tem- ${ }_{21}$ ${ }_{22}$ perature in models of power consumption. Works that tackle this challenge are concerned ${ }_{22}$

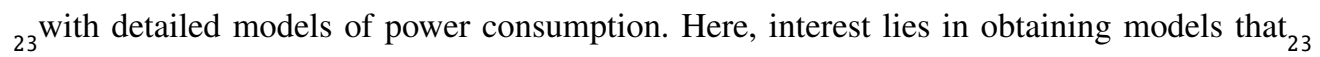
${ }_{24}$ incorporate dependence on hyper-parametric attributes like temperature. 24

25

3.1.7 P7: Loading the VE's resources and measuring resource use 25

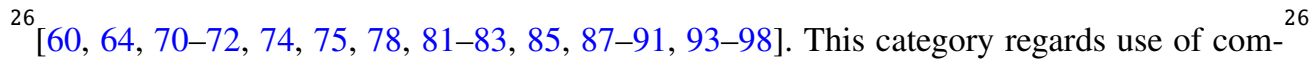

${ }^{27}$ puting resources and measurement of such use by VEs. Interest stems from the role of ${ }^{27}$

${ }^{28}$ resources as predictors in modeling. The researcher is firstly concerned with loading, or ${ }^{28}$

${ }^{29}$ effecting use of resources. What means within the operating context of the $V E$, can be ${ }^{29}$

${ }^{30}$ used to load a resource? Should it be loaded in isolation (using synthetic loads) or should ${ }^{30}$

${ }^{31}$ it be loaded using representative (realistic) workloads? Once these problems have been ad- ${ }^{31}$

${ }^{32}$ dressed, the concern with measurement of resource use arises. The problem here consists ${ }^{32}$

33 f identifying the means that quantify resource use made by the loading. 33

of identifying the means that quantify resource use made by the loading. 


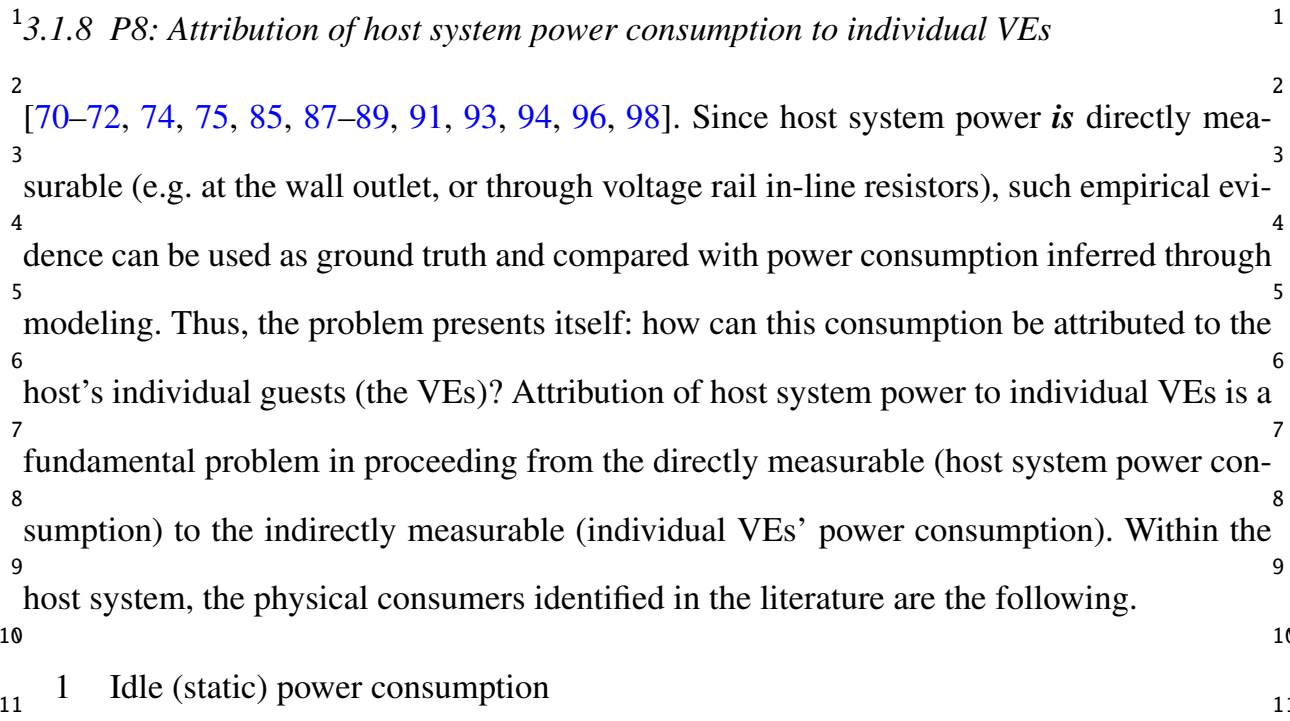

(a) Power consumption while idle is not attributable to any VE at all, as this con- ${ }_{12}$ sumption arises out of electronic behavior of semiconductor material, not of ${ }_{13}$ computation, communication or storage.

(b) Nonetheless, this power consumption must be accounted for and different ap- ${ }_{15}$ proaches have been followed. For example: the physical machine's idle power ${ }_{16}$ is attributed to individual VEs in fractions equal to the ratio of each VE's virtual ${ }_{17}$ CPUs (vCPUs) count to the total complement of vCPUs active on the physical ${ }_{18}$ machine $[70,84-86]$.

2 Active (dynamic) power consumption is required to process a workload

(a) The active component can be linked to a particular VE.

(b) This includes active power consumption in peripherals, e.g. NIC and mass stor- ${ }_{22}$ age device.

3 Overhead, for example:

24

(a) operation of heat dissipating units (fans) to prevent thermal runaway, and 25

(b) losses in the power supply.

A "top" (host) - "down" (guest) approach to attribution has been observed. 27

1 Decide on what host system power consumption is within the scope of the study ${ }^{28}$ and how to divide it. The problem of attribution of the above three causes may be summarized as follows:

(a) Is idle power attributed to the VEs or is it attributed to the host/a privileged ${ }^{31}$ guest?

(b) Is consumption by peripherals within scope? How will this be attributed? 
2

3

54 This fourth consideration is tackled only by those researchers who investigate the ${ }^{5}$

6

7 Implementation of software Layer 2 (L2) data plane switching tion going to account for them?

2 Select a set of performance metrics that are correlated to a VE's power consumption. ${ }^{3}$

3 Select a model that maps a VE's performance metrics to its power consumption. 4 adaptability of the attribution obtained through steps 1,2 and 3. Does the obtained ${ }^{6}$ attribution adapt well to concurrent, co-hosted VEs? That is: if concurrent, co-hosted ${ }^{7}$ VEs were to be investigated, would the division, metrics and model still result in ${ }^{8}$ accurate prediction?

3.1.9 P9: Implementation of virtual I/O; P10: Implementation of network functions; P11:

(c) Are overheads modeled or is correlation with other sources of power consump- ${ }^{1}$ ${ }_{14}[62,63,66]$. These three categories are introduced together, since elements from the re- ${ }_{14}$ ${ }_{15}$ spective categories are commonly implemented as a set for the purpose of realization of ${ }_{15}$ ${ }_{16}{ }^{\text {virtualization of network functions. Here, researchers seek comparative statements and/or }}{ }_{16}$

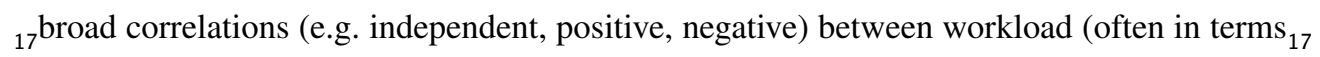
${ }_{18}$ of packet rate and size) and power consumption, across implementations of the same type ${ }_{18}$ ${ }_{19}$ As was observed for categories P1 and P2, researchers seek a profile of the power charac- ${ }_{19}$ ${ }_{20}{ }^{\text {teristics of implementations. It may be helpful to repeat that by "profile", we understand }} 20$ ${ }_{21}{ }^{\text {that }}$ characteristics sought here are not of the detailed form of closed-form expressions. $\quad 21$

22 Examples of elements from the respective categories are: 22

23 - Virtual I/O (P10): virtio [99] and DPDK poll-mode drivers (PMDs) [100] 23

24 - Network functions (P11): Bro (now Zeek) [101] and Snort [102] 24

25 - Software layer 2 data plane switching (P12): Open Virtual Switch (OvS) [103] and ${ }^{25}$

26 VALE [104]. 26

${ }^{27}$ Problems in each of these three categories merit separate classification as they have been ${ }^{27}$

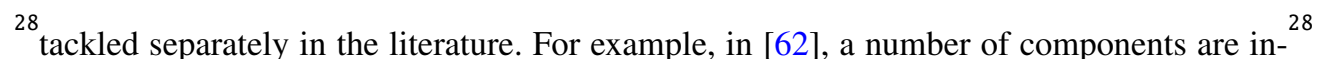

${ }^{29}$ vestigated: three different implementations of software virtual switch (P12), two different ${ }^{29}$

${ }^{30} \mathrm{I} / \mathrm{O}$ virtualization devices and two different implementations of the same network function ${ }^{30}$

${ }^{31}$ (intrusion detection system (IDS)). In [66], power consumption by packet transmission un- ${ }^{31}$

${ }^{32}$ der DPDK is investigated under the condition of enforcement of (a) the network adapter's ${ }^{32}$

33 affinity to NUMA nodes and (b) DPDK process pinning to procssor cores. 33

affinity to NUMA nodes and (b) DPDK process pinning to processor cores. 
${ }^{1} 3.1 .10$ P12: Investigation of processor green capabilities 1

${ }^{2}[57,64,73,80,93-95,97,105]$. Works in this category investigate low-power-idle (LPI) ${ }^{2}$

${ }^{3}$ and adaptive rate (AR) operation of a processor as a means of reducing power consump- ${ }^{3}$

${ }^{4}$ tion. The challenge is broad enough to permit a sub-categorization into (a) those works ${ }^{4}$

${ }^{5}$ that investigate the influence of frequency as a power-model parameter $[93,94]$ and $(b)^{5}$

${ }^{6}$ other works that address improved, real-time governance of LPI and/or AR [64, 95, 105] ${ }^{6}$

${ }^{7}$ to minimize the power consumed to process a load.

${ }^{9}$ 3.1.11 P13: Improvement of power efficiency of high-performance IO virtualization 9

10 frameworks 10

${ }^{11}$ A separate classification was set up for [64] as this work represents an evolution of those ${ }^{11}$

${ }^{12}$ classified under P9. This work extends beyond profiling and suggests use of low-power- ${ }^{12}$

${ }^{13}$ instructions as the means to balance performance and power efficiency. 13

14

${ }^{15} 3.2$ A taxonomy of characteristics of application of the scientific method 15

${ }^{16}$ Fig. 2 illustrates the taxonomy we use to structure the approaches detected in research ${ }^{16}$

${ }^{17}$ work. Line thickness and percentage values represent the utility (see [45, subsection 7.5]) ${ }^{17}$

18 of the specific approach. We now proceed to describe the categories within the context of ${ }^{18}$

19 the taxonomy. Each approach-category's description is preceded by a list of references to ${ }^{19}$

${ }^{20}$ works, each one of which uses a component within that category's set.

22.2 .1 Analytical foundations -22

${ }^{23}$ This group of categories regards the theory and hypotheses that comprise the essential ${ }^{23}$ 24 abstractions at the basis of scientific research.

25

${ }^{26}$ A1: Power attribution principle $\left[62,63,70-72,74,75,82,85,87-89,91,93,94,96{ }^{26}\right.$

${ }^{27} 98$ ]. When system power is measured, whether at the wall outlet or at one or more of

${ }^{28}$ the power supply's output lines, there is the problem of attributing the measurement to ${ }^{28}$

${ }^{29}$ the logical divisions (VEs) of the host computer system. Attribution of system power to ${ }^{29}$

${ }^{30}$ modeled entities starts with a decision on which power consumption is within scope (see ${ }^{30}$

${ }^{31}$ section 3.1.8). Next, power in scope is attributed to (burdened on) one or more entity. ${ }^{31}$

${ }^{32}$ For example: will idle power consumption be attributed to the host system or will it be ${ }^{32}$

33 atributed to the VEs? 33 attributed to the VEs? 
${ }^{1}$ A2: Modeling bias $\quad[64,70-77,85,87-89,91,93,95-97,105]$. Researchers approach the ${ }^{1}$ ${ }^{2}$ problem of developing a model under some bias which conditions their final outcome. This ${ }^{2}$ ${ }^{3}$ bias is manifest in researchers' selection of a particular type of regression to apply to their ${ }^{3}$ ${ }^{4}$ data. We note that this same observation is carried in [106].

5 We delegate our observations on researchers' methods to section 7.2.2. Here, on the other ${ }^{5}$

${ }^{6}$ hand, our purpose is solely to draw attention to what we have observed as researchers ${ }^{16}$

${ }^{7}$ modus operandi without analyzing their choice of approach. ${ }^{7}$

9A3: Green operating principles $[64,95,105]$. Works in this category weave radical (i.e. 9 10 from the grass roots) approaches to power efficiency into their developments. For example10 11instead of conventional scheduling, run-to-completion [107] is exploited to obtain dedi-11 12cated (or, at least, very sparsely shared) resources for the processing of packets. This ap-12 13proach is further nuanced by real-time control of adaptive rates and sleep depth. In one13 14particular case [64], the novel concept of a low-power instruction instead of transitions14 15to/from low-power idle (sleep) states is used. 15 16 16 ${ }_{17}$ A4: Physical analysis [73]. This category regards approaches rooted in physical proper- ${ }_{17}$ ${ }_{18}$ ties of (semiconductor) material in the consumption of energy. For example, [52] regards ${ }_{18}$ ${ }_{19}$ a unique study that implicitly applied Dennard's law in the process of obtaining weights ${ }_{19}$ ${ }_{20}$ that scale a processor sub-unit's contribution to power consumption. In [108], the physical ${ }_{20}$ ${ }_{21}$ cause of power consumption in metal-oxide-semiconductor (MOS) material is examined ${ }_{21}$ ${ }_{22}$ and used as the basis for modeling equations. 22 23 A5: Identification and use of metrics of energy efficiency $\quad[57,58,78,97]$. The relationship between system architecture and power consumption can be investigated through identifi25 cation of relevant metrics of energy efficiency. For example, an easily recognizable metric, 2626 albeit rather broad in possible interpretations, is the $\mathrm{J} / \mathrm{b}$ (joule/bit). Use of such metrics encourages joint consideration of function and power consumption. 28

${ }^{29} 3.2 .2$ Experiment design $\quad 29$

${ }^{30}$ The practical, hands-on aspects of the empirical process are the product of a (probably ${ }^{30}$ ${ }^{31}$ cyclical) design phase, concerned with a number of stewarding activities pertinent to test ${ }^{31}$ ${ }^{32}$ subjects and ancillary objects in the testing scenario, instrumentation, inputs and outputs.

${ }^{33}$ The activities include selection, configuration, interconnection, initiation, observation and ${ }^{33}$ 
${ }^{1}$ termination. We have identified several examples of such activities within our research ${ }^{1}$

${ }^{2}$ scope and grouped them under resource provision (categories A6, A7), workload selection ${ }^{2}$

${ }^{3}$ (A8 - A9) and data collection (A10 - A13). We describe these categories next.

4

${ }_{5}$ A6: Managed resource provision [57-60, 62, 63, 66, 73, 79-81, 85, 93-95]. This con-5 ${ }_{6}$ cerns the provision of resource capacity either to a specific VE (the guest system) or to the ${ }_{6}$ 7physical entity (the host system) hosting the VEs. Within the empirical process, the tech-7 ${ }_{8}$ niques in this category provide the means to observe the effect on power consumption of $f_{8}$ gmanaged changes in resource provision. Examples include (manual) pre-configuration of: 9

10 - frequency of operation of processor cores [57, 63, 93]; 10

11 - core affinity $[59,66]$ and hardware-thread affinity [81]; 11

12 - network interface data rate capacity capping [79]. 12

13 These techniques are executed as part of the process of setting up experimentation, before $_{13}$ ${ }_{14}$ Operations start. This qualification is necessary to distinguish from such approaches as may ${ }_{14}$ ${ }_{15}$ change the operating, run-time context. 15

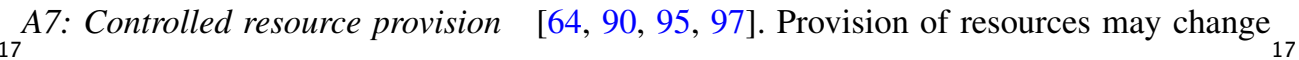
${ }_{18}$ during the running of an experiment (rather than before it starts). Approaches in this category include the automated adjustment of:

1 processor frequency (also known as performance state, or P-state) [64, 90, 95, 97]

2 depth of processor sleep (also known as as low-power-idle-state, or C-state) [95]

3 number of hardware threads [90]

4 time spent running a low-power instruction [64]

These techniques are approaches to solving the problem of full-throttle operation. Without ${ }_{25}$ guided operation of adjustments like those listed above, operation of the processor may ${ }_{25}$ ${ }_{26}$ quite reasonably be likened to a multi-assembly-line manufacturing plant that operates line ${ }_{26}$ machinery whether there are goods to produce or not.

${ }^{28}$ A8: Resource-specific workload, A9: Representative workloads These two categories re- ${ }^{28}$ ${ }^{29}$ gard the workload selection stage, within experiment design in the scientific method. The ${ }^{29}$ ${ }^{30}$ workload comprises the inputs referred to earlier (section 3.2.2). In Table 6, we identify ${ }^{30}$ ${ }^{31}$ workload type as one of the seven dimensions of variability of power models. The influence ${ }^{31}$ 32 of workload type on the model obtained is evident in the attention paid by researchers to ${ }^{32}$ 33 selection of workload type. We can distinguish two broad categories of type. 
1 Resource-specific workloads (category A8) [57-60, 62, 63, 66, 72-74, 76, 77, 79, 80, 82, ${ }^{1}$

$\left.{ }^{2} 83,87-89,96,98\right]$ are applied to investigate the impact of utilization of specific resources ${ }^{2}$

${ }^{3}$ on power consumption. Such synthetic workloads are applied to a machine (whether virtual ${ }^{3}$

${ }^{4}$ or physical) to reduce (as much as possible) the scope of power-consuming resources to $\mathrm{a}^{4}$

${ }^{5}$ targeted set. Resource-specific workloads are most commonly used in exploratory work, to ${ }^{5}$

${ }^{6}$ gain an understanding of the behavior of a resource's power consumption. We refer to this ${ }^{6}$

${ }^{7}$ approach as resource-specific workloads, or synthetic workloads, or resource isolation. ${ }^{7}$

8 Representative workloads (i.e. representative of real use) (category A9) [60, 63, 64, 70, ${ }^{8}$

$\left.{ }^{9} 71,73,75,78,81,82,85,87,90,91,93-97\right]$ may be used complementary with, or alterna- ${ }^{9}$

${ }^{10}$ tive to, resource-specific (synthetic) workloads. A notable complementary use is made in ${ }^{10}$

${ }^{11}$ the testing (post-training) phase of model development, when representative workloads are ${ }^{11}$

${ }^{12}$ used to validate a model (obtained using synthetic workloads). They may also be used in an ${ }^{12}$

${ }^{13}$ entirely alternative approach to synthetic loading, to support development of application- ${ }^{13}$

${ }^{14}$ agnostic models. Representative workloads lead to training data that incorporates variation ${ }^{14}$

${ }^{15}$ in utilization of more than one resource at a time; hence providing at least limited applica- ${ }^{15}$

${ }^{16}$ tion agnosticism. 16

$17 \quad 17$

${ }^{18}$ A10: Resource instrumentation (microarchitecture and system software), A11: Load in- ${ }^{18}$

${ }^{19}$ strumentation: direct characterization, E measurement of workload, A12: Direct power ${ }^{19}$

${ }^{20}$ measurement, A13: Hardware instrumentation of predictors These four categories regard ${ }^{20}$

${ }^{21}$ the data collection stage, within experiment design in the scientific method. Previous cate- ${ }^{21}$

${ }^{22}$ gories (section 3.2.2) regard selection of workload type. Approaches described here regard ${ }^{22}$

${ }^{23}$ measuring how much of a resource is being used, or workload is being applied. Categories ${ }^{23}$

${ }^{24} \mathrm{~A} 10, \mathrm{~A} 11$ and A13 regard instrumentation of those variables considered (by the researchers ${ }^{24}$

${ }^{25}$ concerned) to be reliable predictors of power consumption. 25

${ }^{26}$ Category A10 [58, 60, 63, 64, 70-72, 74-77, 82, 85, 87-91, 93-98] includes approaches ${ }^{26}$

${ }^{27}$ that measure resource use. These measurements are then used to predict power consump- ${ }^{27}$

${ }^{28}$ tion. We make a somewhat weak distinction, for reasons we shall refer to, between in- ${ }^{28}$

29 strumentation of microarchitecture and instrumentation by system software. The former ${ }^{29}$

30 gards parts of the processor interface that address the processor's infrastructure for mon 30 regards parts of the processor interface that address the processor's infrastructure for mon-

${ }^{31}$ itoring: event counters (e.g. instructions retired, last-level-cache (LLC) misses, translation ${ }^{31}$

${ }^{32}$ lookaside buffer misses) and, more recently power counters (e.g. Intel's Running Aver- ${ }^{32}$

${ }^{33}$ age Power Limit (RAPL) [109]). On the other hand, system software's instrumentation ${ }^{33}$ 
${ }^{1}$ is carried out through intermediary system software and includes, most notably, proces- ${ }^{1}$

${ }^{2}$ sor utilization. We have seen references to these two categories as "hw counters" and "sw

${ }^{3}$ counters" respectively [110]. The distinction is weak since system software is increasingly ${ }^{3}$

${ }^{4}$ exposing microarchitecture instrumentation (consider, for example, Linux's perf tool). This ${ }^{4}$

${ }^{5}$ reduces the need to directly access hardware registers and blurs the separation between ${ }^{5}$

${ }^{6}$ what is abstracted and what is concrete, raw, hardware data. Nonetheless, through our ${ }^{6}$

${ }^{7}$ understanding of the data used, we have been able to separate the approaches into two ${ }^{7}$

${ }^{8}$ sub-categories.

9 Category A13 regards use of hardware sensors to obtain inputs and/or parameters for the ${ }^{9}$

${ }^{10}$ power model $[64,73,93,94]$. These include: 10

11 voltage sensors (processor supply voltage, VDD) 11

$\begin{array}{lll}12 & 2 & \text { temperature sensors (processor package and memory temperatures) } \\ & 12\end{array}$

$13 \quad 3$ fan speed sensors (processor and chassis fans) 13

144 wall-clock time measurement 14

${ }^{15}$ Some of these variables are used in models that predict power consumption while account- ${ }^{15}$

${ }^{16}$ ing for the effect of drift of temperature and automated supply voltage adjustment (in dy- ${ }^{16}$

${ }^{17}$ namic voltage and frequency scaling - DVFS). 17

18 Category A11 regards use of a simple characterization of workload as predictor of power ${ }^{18}$

${ }^{19}$ consumption [58, 62-64, 77, 78, 80, 81, 83, 90, 95, 97]. This is notably different from ${ }^{19}$

${ }^{20}$ approaches in category A10, which are concerned with resource use as predictor of power ${ }^{20}$

${ }^{21}$ consumption. Other examples (apart from those given earlier) of workload metrics as inputs ${ }^{21}$

${ }^{22}$ are "number of processes" [83] (from the same process image, say), "transmission rate" 22

${ }^{23}$ [81] and millions of instructions per second (MIPS) [77]. Characterizations may need to ${ }^{23}$

${ }^{24}$ be sharper. For example, since packet network traffic arrival is known to often have the ${ }^{24}$

${ }^{25}$ properties of a Batch Markov Arrival (stochastic) Process (BMAP), this is an operating ${ }^{25}$

${ }^{26}$ constraint adopted in the approach of several works studying power efficiency of network ${ }^{26}$

${ }^{27}$ functions $[64,95,105,111] . \quad 27$

${ }^{28}$ Category $\mathbf{A 1 2}$ refers to the measurement of power consumption, which is most usually ${ }^{28}$

${ }^{29}$ measured at the wall outlet or at the power supply inlet $\left[57-60,62,63,70-72,74-77,{ }^{29}\right.$

$\left.{ }^{30} 80,81,83,85,88,91,96\right]$. More granular approaches are desirable, and indeed we do ${ }^{30}$

${ }^{31}$ find cases (e.g. [75]) that attempt measurement at the power supply output. The principal ${ }^{31}$

${ }^{32}$ drawback of such granular techniques is not construction of intermediary hardware (e.g. ${ }^{32}$

${ }^{33}$ riser boards, or line resistors) but the difficulty in attributing power drawn through any ${ }^{33}$ 
${ }^{1}$ single dc voltage output (or group thereof) to particular consumers. With the advent of ${ }^{1}$

${ }^{2}$ RAPL and certain guarantees on its accuracy, the need for direct power measurement has ${ }^{2}$

${ }^{3}$ been, at least partially, avoided.

3

5.2.3 Model validation

${ }^{6}$ Model validation is a multi-faceted endeavor and this is reflected in the approaches we have ${ }^{6}$

${ }^{7}$ detected. The approaches range across the candidates that would typically be considered: ${ }^{7}$

${ }^{8}$ simulation (A14) [64], use of test data (A15) [70, 75-77, 89, 97] and corroboration through ${ }^{8}$

${ }^{9}$ experimentation (A16) $[64,95]$. We skip elaborating on these categories as they are either ${ }^{9}$

${ }^{10}$ self-evident (A15) or because they are too rarely used to permit general commentary. How- ${ }^{10}$

${ }^{11}$ ever, to these three categories we add a fourth (A17), namely: model adaptation technique, ${ }^{11}$

${ }^{12}$ which we describe below and explain why it fits within this branch of the taxonomy. 12

13

${ }^{14}$ A17: Model adaptation technique This refers to the approach(es) taken (if any) to de- ${ }^{14}$

${ }^{15}$ velop an adaptable model or modeling system $\left[70-72,74,75,80-83,85,87,88,90,91,{ }^{15}\right.$

$\left.{ }^{16} 93,94,96,98\right]$. Here, adaptability refers to the fitness for use which the model exhibits ${ }^{16}$

${ }^{17}$ under change in one or more of the seven dimensions of variability that will be defined ${ }^{17}$

${ }^{18}$ in detail in Section 4.1.1 below (see table 6). Model adaptability is essential for practical ${ }^{18}$

${ }^{19}$ virtualization, where changes in, say, the number of co-hosted, concurrent VEs, or in work- ${ }^{19}$

${ }^{20}$ load type are commonplace. Below, we list the major approaches taken towards producing ${ }^{20}$

${ }^{21}$ adaptable models. Since these approaches emerge in the context of validating a model's ac- ${ }^{21}$

${ }^{22}$ curacy under some limited range of the seven-dimensional space of operating conditions, ${ }^{22}$

${ }^{23}$ we classify this category of approaches as an aspect of model validation. 23

24 Adaptation to change in the number of co-hosted, concurrent VEs: is widely ${ }^{24}$ 25 achieved through time-division multiplexing of event counters $[72,75,82,91,98],{ }^{25}$

26 RAPL counters and CPU utilization [74, 85]. This approach enables use of such met- ${ }^{26}$

27 rics as predictors of dynamic (active) power consumption, by apportioning counts to ${ }^{27}$

$28 \quad$ VEs in accolate 28 VEs in accordance with the time during which the VEs were active.

29 Adaptation to uncorrelated causes of power consumption: might be achieved ${ }^{29}$ through additional predictors [94], to follow causes of power consumption that do 30 not correlate well with counters within the current set. This case reflects itself as ${ }^{31}$ poor accuracy in predicted power consumption. Although counter-based models are ${ }^{32}$ reported to fit a variety of processor- and memory-intensive workloads well, it may ${ }^{33}$ 
be necessary to account for unanticipated activity through the approach of adding ${ }^{1}$ previously unused counters.

3 It may not be possible to fit a single model with parameters known a priori to the ${ }^{3}$ whole range of inputs within the scope of study. The commonality here is that no ${ }^{4}$ single representation of power can be obtained, notwithstanding the diversity of pre- $^{5}$ dictors employed in this pursuit. The following adaptive techniques have been found ${ }^{6}$ in the literature.

(a) Dataset partitioning, where the dataset is of the form $\{(\{\text { predictors }\} \text {,response })\}^{\beta}$, is used in [89] to match the best model out of a set of models to current, actual ${ }^{9}$ operation. An individual model in the set is associated with a single node in $\mathrm{a}^{10}$ decision tree and the node is selected according to features pertinent to current, ${ }^{11}$ actual operation. A simpler, but conceptually similar, approach is taken in [71]. ${ }^{12}$ A number of models are devised and model-selection features are limited to ${ }^{13}$ the number of active VMs and a coarse grading of CPU utilization. 14

(b) Modeling on demand is the term we use to succinctly refer to the fourth adap- ${ }^{15}$ tation class of techniques.

i. One early example of this approach is found in [87], where the depen- ${ }^{17}$ dence of the model on workload has been addressed through online train- ${ }^{18}$ ing, whenever prediction accuracy of extant models falls out of a range of ${ }^{19}$ tolerance. The rationale adopted is that if model adaptation to such an "un- ${ }^{20}$ seen" case is limited to parametric tuning, then a modeling system might ${ }^{21}$ be able to construct a model while VEs are in operation.

ii. A broader perspective is found in [90]. An automated system for pro- ${ }^{23}$ filing containerized applications is described and demonstrated. Con- ${ }^{24}$ tainerized applications are profiled from three perspectives: computing re- ${ }^{25}$ sources consumed, energy consumed and performance. In this case, the ${ }^{26}$ rationale is that energy consumption can be optimized by determination ${ }^{27}$ of a frequency-and-hardware-threads host configuration that meets per- ${ }^{28}$ formance requirements. Thus: starting from central functional require- ${ }^{29}$ ments (performance requirements), operating conditions are determined ${ }^{30}$ that minimize energy consumption. This approach is capable of meet- ${ }^{31}$ ing the challenges posed by heterogeneous host hardware and application ${ }^{32}$ (workload) diversity, at the cost of analytical modeling. Indeed, charac- ${ }^{33}$ 
${ }_{5}$ Developments fall cleanly into one of two groups: (a) models of power consumption and (b) ${ }_{6}$ observations on dependencies of power consumption. The first group (D1-D10) includes ${ }_{6}$ ${ }_{7}$ developments that predict power consumption over a sub-space of the seven-dimensional ${ }_{7}$ ${ }_{8}$ space of operating conditions. The second group (D11 - D18) includes developments that ${ }_{8}$ ${ }_{9}$ are oriented towards the correlation of power consumption with aspects of system integra- ${ }_{9}$ ${ }_{10}$ tion. As the taxonomy is rather broad, we present it in three parts:

11 Fig. 3: top-level fork into models and dependencies.

11

122 Fig. 4: the taxonomy of models.

$13 \quad 3$ Fig. 5: the taxonomy of dependencies.

${ }^{14} 3.3 .1$ Models of power consumption

${ }^{15}$ Models of host system power consumption: D1: Linear regression models; D2: Non-linear ${ }^{15}$

${ }^{16}$ regression models; D3: Machine-learnt models and D4: Models of local mass storage ${ }^{16}$

${ }^{17}$ These four categories regard developments in host system power models, under the condi- ${ }^{17}$

${ }^{18}$ tion where workload is processed by VEs. This contrasts with the scope of developments ${ }^{18}$

${ }^{19}$ referred to under categories D5, D6 and D7, where power consumption by a VE is mod- ${ }^{19}$

${ }^{20}$ eled. Such models are interesting from the perspective of analyses of sets of hosted VEs ${ }^{20}$

${ }^{21}$ that seek to identify operating conditions of optimal host power efficiency. As predictors, ${ }^{21}$

2222 such analyses use instrumentation that measures resources used by the VEs.

23 Categories D1, D2 and D3 all predict power consumption in terms of resource use but ${ }^{23}$

24

differ in the type of model produced.

- D1 regards models of power consumption through linear combinations of scalar pre- ${ }^{25}$

26 dictors [70-72, 82, 87]. The scalar predictors are resource usage metrics.

27 - D2 regards polynomial or simple mathematical powers of resource use (the scalar ${ }^{27}$ predictors) $[70,73,74,88,93]$.

- D3 regards models that employ machine learning (e.g. Gaussian Mixture, Support ${ }^{29}$ Vector Machine, Neural Networks) [75, 89, 91]

31 Category D4 regards models of power consumed by mass storage local to the host sys- ${ }^{31}$ ${ }^{32}$ tem [98]. These models attempt to predict power consumption in terms of activity metrics ${ }^{32}$ ${ }^{33}$ such as total amount of time spent in a known state (in terms of power consumption, e.g. ${ }^{33}$ 
${ }^{1}$ active/idle), rate of data exchange (MB/s or input/output operations per second) and mode ${ }^{1}$

${ }^{2}$ of operation (sequential/random and read/write). In the context of the approximations ob- ${ }^{2}$

${ }^{3}$ served in the development of these models, their accuracy cannot be fairly assessed without ${ }^{3}$

${ }^{4}$ implementation. Our choice of a separate category for this device is not as arbitrary as the ${ }^{4}$

${ }^{5}$ first glance might suggest. Few works extend into meaningful consideration of I/O. As edge ${ }^{5}$

${ }^{6}$ computing takes shape, the share of power consumption attributed to I/O devices can fairly ${ }^{6}$

${ }^{7}$ be expected to increase.

${ }_{10}$ Adaptable VE models: D5, D6 and D7 Developments within these categories consist of ${ }_{10}$ ${ }_{11}$ adaptable models of the virtualized entity's power consumption and have two important ${ }_{11}$ ${ }_{12}$ characteristics in common.

131 They are adaptable in the seventh dimension of adaptability (see Table 6): within the 13

14 scope of the employed workload, these models can predict power consumption by 14

15 multiple concurrent, co-hosted (active on a single host system) VEs. 15

162 The predictors are the measured amount of used computing resources. 16

17 Models can be distinguished by the predictors they use, workloads employed and mod- ${ }^{17}$

${ }^{18}$ eling approach: $\quad 18$

191 predictors (of VE power consumption) are obtained from system software's instru- ${ }^{19}$ 20 mentation, e.g. CPU utilization (see section 3.2.2), and from microarchitecture in- ${ }^{20}$

21 strumentation, e.g. LLC misses (see section 3.2.2);

2 workloads used to obtain the model (this restricts the range of workloads within ${ }^{22}$ which the model is valid) may be:

(a) specific workloads: the most restrictive, as they relate to a particular test load; ${ }^{24}$

(b) synthetic workloads: less restrictive than specific but limited to exercise of one ${ }^{25}$ resource, typically the CPU;

(c) combinational workloads: still less restrictive, involving the exercise of a num- ${ }^{27}$ ber of resources of the host system (e.g., SPEC CPU benchmarks may be both ${ }^{28}$ processor and memory intensive); $\quad 29$

(d) representative workloads (e.g. TPC-W [112]) produce models that are readily ${ }^{30}$ associated with use cases. 
2

${ }^{27}$ point, or change in power consumption over some operating range. Examples follow. $\quad 27$ mic regressions $[74,79,88]$;

2

(c) category D7: machine-learnt (combinations of resource use) [75, 91].
Models of power consumption that use workload profile as predictors: D8 and D9 Categories D8 and D9 group developments from (two) sets of RUs that predict power consumption of hosts and/or VEs through (measurement of) some characteristic of the submitted workload. This contrasts with RUs in categories D1 - D7, where prediction is obtained hrough (measurement of) some computing resource (processor and/or memory and/or ${ }_{10}$ $\mathrm{I} / \mathrm{O})$. Most developments in this category are obtained through abstraction of hardware by one or more model parameters that express power consumption under case-specific conditions of operation. Some of these abstractions are identified in the descriptions of these two

14

Developments in D8 [64, 76-81, 83, 90, 95, 97, 105] use: 15

1 processing load (number of processes, millions of instructions per second (MIPS),16 etc.) pertaining to a specific application, as predictors of host system power [76-17 80, 83];

18

2 packets per second, through an intrusion detection system implemented in a VNF19 [97];

3 transcoded frames per second, through a transcoder implemented in a containerized 21 network function (CNF), and inferred images per second, also in a CNF [90]; $\quad 22$

4 average network transmission rate, as a predictor of host system power [81] 23

5 statistics of a Batch Markov Arrival Process (BMAP) (packet traffic) as a means of 24 prediction of power consumption by a VNF $[95,105]$

Hardware is abstracted through measurement of power consumption at some operating ${ }^{26}$

1 In [78], where energy efficiency of web interactions is studied, the operating point is ${ }^{28}$ an entire VM running the TPC-W benchmark [112].

2 In [80, 83], the operating points are the host's power consumption when (a) idle, (b) ${ }^{30}$ one core is active (processing load) and (c) maximum, with all cores active. Fur- ${ }^{31}$ thermore, use is made of the step increment in consumption corresponding to the ${ }^{32}$

(b) category D6: power (integer and non-integer powers), exponential and logarith- ${ }^{1}$ activation of each additional core. Cores are activated when they are utilized by VEs. ${ }^{33}$ 
13 In [81], the operating point is the power consumption when the host is not transmit- ${ }^{1}$

2 ting. An affine relationship with the host's transmission rate (transmissions originate ${ }^{2}$

3 on hosted VEs) is found.

44 In [77], an operating range is used: the increase in power consumption that corre- ${ }^{4}$

5 sponds to an increase in MIPS on the VEs. 5

65 In [95], the operating point is the power consumption when a VE running on a single ${ }^{6}$

7 processor core is switching packets at the maximum rate for a given performance ${ }^{7}$ state.

8

96 In [97], fifty-four (54) different features of network traffic are input to an artificial $^{9}$ neural network that selects the operating frequency that optimizes power consump- ${ }^{10}$ tion. processor's internals (to obtain predictors of power consumption). The exception is [95]; 
1 Notwithstanding the origin of these observations in modelling work, it may be argued ${ }^{1}$

${ }^{2}$ that they might also be classified within the dependencies branch of the taxonomy. $\mathrm{We}^{2}$ ${ }^{3}$ have chosen the models branch, but as further studies add to the body of knowledge on ${ }^{3}$ ${ }^{4}$ how to operate processor green capabilities, this category's position in the taxonomy might ${ }^{4}$ ${ }^{5}$ need to be changed.

${ }_{7}$ D10: Relative accuracy of modeling approaches $\quad[73,75,76,89]$ Developments presented sunder category D10 are comparisons of the relative accuracy of alternative modeling meth-8 ${ }_{9}$ ods with respect to conventional polynomial (including linear), power, exponential and 9 ${ }_{10}$ logarithmic regression. These developments have been found within works that show mod-10 ${ }_{11}$ els classified under category D9. The purpose is to qualify and quantify improvements of ${ }_{11}$ 12 machine-learnt models with respect to conventional regression models. 12 13

3.3.2 Dependencies of power consumption 15

This parent node of the taxonomy is divided into two child nodes that are not strictly mutually exclusive. For example, the software data plane is considered in works under D15. 16 . ${ }_{17}$ Clearly, the software data plane is a logical artifact and might be included within a child ${ }_{17}$ node of "physical and logical artifacts", or directly thereunder as a leaf node (i.e. as a category). The choice of separation of D15 - D18 and inclusion under parent node "networking workloads" was taken for two principal reasons. Firstly, the recurrence of investigation of ${ }_{21}$ power consumption's dependency on networking workload merits attention through sep- ${ }_{21}$ ${ }_{22}$ aration. Secondly, as this survey caters for an audience with an interest in softwarized ${ }_{22}$ networking, an emphasis on power dependency on networking workload seems justified. 23

${ }^{24}$ D11: Knowledge about dependency of power consumption on specific hardware; D12: de- ${ }^{24}$ ${ }^{25}$ pendency of power consumption on architecture; D13: dependency of power consumption ${ }^{25}$ ${ }^{26}$ on resource provisioning and D14: dependency of power consumption on virtualization ${ }^{26}$ ${ }^{27}$ genre E technology Categories D11 - D14 are grouped into a set of works that obtain the ${ }^{27}$ ${ }^{28}$ sense of the correlation (positive/negative/none) between power consumption and some ${ }^{28}$ ${ }^{29}$ genre of artifacts: specific hardware types (D11), computer architecture (D12), resources ${ }^{29}$ $30 \quad 30$ provided (D13) and virtualization genre and technology (D14).

31 Category D12 groups works that relate to observations on the impact of architectural ${ }^{31}$ ${ }^{32}$ features on power consumption [57, 58, 80, 83, 88]. While these observations are useful, ${ }^{32}$ ${ }^{33}$ they are generally too coarse to be directly applicable to real-time power control. Their ${ }^{33}$ 
${ }^{1}$ use emerges from guidance which they provide in the development of power models. For ${ }^{1}$

${ }^{2}$ example, it was observed that when the number of threads that fully occupy a core's time ${ }^{2}$

${ }^{3}$ (active $100 \%$ ) exceeds the number of logical cores in the system, the energy efficiency ${ }^{3}$

${ }^{4}$ (measured, in this case, in hash/J, a computational metric of energy efficiency) decreases ${ }^{4}$

${ }^{5}[57]$. Evidently, this is good guidance; equally evidently, it is not a directly applicable ${ }^{5}$

${ }^{6}$ development.

7 Categories D11, D13 and D14 relate closely to (various aspects of) implementation, and ${ }^{7}$

${ }^{8}$ as such are of particular interest to the system integrator. Data of high quality from these ${ }^{8}$

${ }^{9}$ developments inform and guide the tasks of gathering components into systems that meet ${ }^{9}$

${ }^{10}$ the non-functional requirements obtained from concern with energy and power efficiency. ${ }^{10}$

11 Category D11 gathers observations about the dependency of power consumption on spe- ${ }^{11}$

${ }^{12}$ cific processor hardware $[58,82,88]$. We have observed that these developments are gath- ${ }^{12}$

${ }^{13}$ ered as a by-product of the process of research; they are rather incidental. Like any imple- ${ }^{13}$

${ }^{14}$ mentation, their usefulness is limited to the lifetime of the concerned device(s). ${ }^{14}$

15 Category D13 [85] includes developments that regard the specific resource configuration ${ }^{15}$

${ }^{16}$ of: $\quad 16$

171 the instantiating host, i.e. the relationship between a VE's power consumption and ${ }^{17}$

18 the specific resources of its host hardware specifics such as the number of cores and ${ }^{18}$

19 amount of memory carried by the host instance;

202 the guest VE, i.e. the variation of a VE's power consumption with its resource as- ${ }^{20}$ signment, on the same host.

22 Category D14 gathers observations about the dependency of power consumption on in- ${ }^{22}$

${ }^{23}$ stances of virtualization genre and technology $[59,60]$. Developments in this category are ${ }^{23}$

${ }^{24}$ less incidental than those in category D2 and are obtained with the focused intention of ${ }^{24}$

${ }^{25}$ tackling challenges relating power consumption of implementations. These developments ${ }^{25}$

${ }^{26}$ relate to a less diversified group of implementations. For example, there are fewer virtu- ${ }^{26}$

${ }^{27}$ alization platforms than processors to choose from. Direct use of these developments is ${ }^{27}$

${ }^{28}$ mostly limited to the specific implementations concerned; however, some generalizable ${ }^{28}$

${ }^{29}$ conclusions exist. For instance, it was observed that both hardware-assisted virtualization ${ }^{29}$

${ }^{30}$ and paravirtualization are less efficient (in the specific empirical setup) than non-virtualized ${ }^{30}$

${ }^{31}$ operation in the use of processor caches [60]. This empirical evidence favours the hypothe- ${ }^{31}$

${ }^{32}$ sis that cache hit ratios suffer due to the greater thread rotation in virtualized and container- ${ }^{32}$

33. ized environments. 
${ }^{1}$ D15, D16, D17 and D18: Knowledge about dependency on power consumption while pro- ${ }^{1}$

${ }^{2}$ cessing a networking workload Development categories D15, D16, D17 and D18 are ${ }^{2}$

${ }^{3}$ grouped here as their central characterization is knowledge about the behavior of power ${ }^{3}$

${ }^{4}$ consumption by VEs while processing a networking workload. 4

5 Categories D15, D16 and D17 reflect the challenges described in P11, P9 and P10, re- ${ }^{5}$

${ }^{6}$ spectively. Category D15 regards contributions to knowledge about the power consump- ${ }^{6}$

${ }^{7}$ tion of software data planes [62, 63]; D16 regards virtualization of network I/O [62, 66], ${ }^{7}$

${ }^{8}$ and D17 is about network functions [62, 63, 97]. Thus, in [66], the power efficiency of ${ }^{8}$

${ }^{9}$ DPDK PMDs is demonstrated with respect to Netmap drivers, for packet transmission. ${ }^{9}$

${ }^{10}$ This development is balanced by [63], where the power efficiency of transmission through ${ }^{10}$

${ }^{11}$ a DPDK-enhanced Open vSwitch is shown to be worse (@500-byte Maximum Transfer ${ }^{11}$

${ }^{12}$ Unit (MTU)) than that of the unenhanced Open vSwitch. In each of these categories, ef- ${ }^{12}$

${ }^{13}$ forts are made to allocate burden through isolation of power consumption and attribution ${ }^{13}$

14 to the sub-system (data plane/virtualized IO/network function) under study. 14

15 Category D18's developments differ from those of D15 - 17, because they cut across these ${ }^{15}$

${ }^{16}$ categories' sub-system boundaries [60, 78]. For example, in [78], the energy efficiency ${ }^{16}$

${ }^{17}$ of web transactions executed on co-located VMs is found to be highest in the operating ${ }^{17}$

${ }^{18}$ condition of processor-core over-subscription (more VMs than cores). In [60], it is shown ${ }^{18}$

${ }^{19}$ that power consumed by packet delivery to a VM through a software packet switch is ${ }^{19}$

${ }^{20}$ much higher than that required for delivery in a non-virtualized environment. In both these ${ }^{20}$

${ }^{21}$ papers, the object of interest incorporates virtualization of network I/O and the data plane. ${ }^{21}$

${ }^{22}$ In [78], the object of interest further encompasses the network function (a web service and ${ }^{22}$

${ }^{23}$ accompanying application and database components). 23

$24 \quad 24$

All four categories are of keen interest to the system integrator. Emphasis is on the com- ${ }^{24}$

${ }^{25}$ ponents in the integrator's set of building blocks, specifically on the behavior of power ${ }^{25}$

${ }^{26}$ consumption of various implementations, and types thereof (in the scope of the categories). ${ }^{26}$ 27

${ }^{28} 3.4$ P-A dyads graphics 28

${ }^{29}$ Over the following pages, we present a comprehensive set of graphics (Figs. 6 and $11^{29}$

${ }^{30}$ to 22) that illustrate the approaches detected to tackle specific challenges (reference num- ${ }^{30}$

${ }^{31}$ bers for pertinent RUs appear in the shape tags). For example, consider Fig. 6 . The graphic ${ }^{31}$

${ }^{32}$ shows the component approaches applied to discover "the impact of specific architectural ${ }^{32}$

33 attributes of the host system on power consumption of VEs" (see P1 in section 3.1.1). The ${ }^{33}$ 
${ }^{1}$ presence of approach categories A1, A5, A6, A8 and A10 - A12 does not mean that $\boldsymbol{e v}{ }^{1}$

${ }^{2}$ ery $\boldsymbol{R U}$ tackling this challenge uses a component from all of the approach categories. $\mathrm{It}^{2}$

${ }^{3}$ does, however, mean that every RU tackling P1 uses a subset of the approaches shown. $\mathrm{We}^{3}$

${ }^{4}$ proceed by giving examples (relevant to P1), with references. 4

51 Managed resource provision (A6): Prior to running experiments, researchers set the ${ }^{5}$

$6 \quad$ conditions for the experiment through this approach $[57,58,63,66]$. 6

72 Resource-specific workload (A8): This may be used to stress the component imple- ${ }^{7}$

8 menting the architectural attribute under test $[57,58,63,66]$. 8

93 Simple workload characterization and instrumentation (A11): The workload must ${ }^{9}$

10 be characterized by some parameter that serves to measure its demand for power ${ }^{10}$

$11 \quad[58,63]$ $[58,63]$.

124 Resource instrumentation (A10): This is an alternative to use of workload profiling ${ }^{12}$

13 (A11) as predictor of power consumption. Rather than use, say, number of threads, ${ }^{13}$

14 or transmit bandwidth (for networking workloads) as predictors, this approach uses ${ }^{14}$

15 resource instrumentation $[58,63]$. 15

165 Identification of metric of energy efficiency (A5): In certain cases [57, 58], energy or ${ }^{16}$

17 power efficiency is investigated, rather than energy or power consumption. In these ${ }^{17}$

18 cases, the researchers identify and use a relevant metric of efficiency, rather than ${ }^{18}$

19 metrics of consumption (watts or joules). 19

20 On any of the dyad graphics, the approaches shown (inside the approach categories) in $^{20}$

${ }^{21}$ clude only those which are used by at least one RU that tackles the challenge category that ${ }^{21}$

${ }^{22}$ is the root of the dyads. We delegate a repository with a full tabulation of all approaches in ${ }^{22}$

${ }^{23}$ any approach category. Similarly, all individual developments within a category are dele- ${ }^{23}$

24

gated to the same repository. See section 7.2.2 for the details.

${ }^{26} 3.5$ Causality DAG 26

${ }^{27}$ The Causality Directed Acyclic Graph (DAG) in Fig. 7 shows a bird's eye-view of the ${ }^{27}$

28 proceedings of research in scope.

29

${ }^{30} 3.6$ Triads Graphic

${ }^{31}$ Fig. 8 shows the triads graphic. To improve readability, we limit illustration to the triads ${ }^{31}$ ${ }^{32}$ that are in the top twenty percentile of a list ordered according to thickness. These triads ${ }^{32}$

33 comprise $49.2 \%$ of the total number of compiled triads. 
${ }^{1} 3.7$ Statistics

${ }^{2}$ Bar charts that illustrate the category metrics described in [45, section 7] are presented ${ }^{2}$ ${ }^{3}$ below.

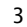

41 Challenges: Fig. 9

5 (a) frequency of occurrence in the set of all RUs;

6 (b) frequency of occurrence in the set of all challenges in all RUs; 6

$\begin{array}{ll}7 & \text { (c) frequency of occurrence, weighted by approach diversity, in the set of all chal- }{ }^{7}\end{array}$ lenges in all RUs.

92 Approaches: Fig. 10

10

(a) frequency of occurrence in the set of all approaches in all RUs;

(b) frequency of occurrence in the set of all triads in all RUs.

123 Developments: frequency of occurrence in the set of all developments in all RUs: ?? ${ }^{12}$

${ }^{13}$ To improve legibility, the $\mathrm{x}$-axis labels show category numbers only. The codes ("terse, ${ }^{13}$

${ }^{14}$ dense representations of a verbose articulation of a concept", see [45, section 3.1] linked to ${ }^{14}$

${ }^{15}$ the numbers are shown in Table 3 , Table 4 and Table 5. In Table 3, we also show questions ${ }^{15}$

${ }^{16}$ that help to clarify articulation of the challenge posed. 16

17

183.7.1 Other results

17

${ }_{19}$ Average number of challenges tackled per RU, $\frac{R_{P_{k}}}{F_{P_{k}}}=2.6$.

18

20

4 Analysis Through Themes

21

The themes presented in this section are the product of a thematic analysis undertaken according to the method described in [45] and summarized in [45, section 10.2]. We first ${ }_{24}$ present an overview through a graphic (Fig. 23) that groups the themes and then proceed to an exposition of the themes within the sub-sections.

25

${ }^{26} 4.1$ State of the art $\quad 26$

${ }^{27} 4.1 .1$ Seven dimensions of variability 27

${ }^{28}$ Through our collation and resolution of the derivatives of the core challenge in this research ${ }^{28}$

29 space, we observe seven dimensions of variability in modeling power consumption by a ${ }^{29}$

${ }^{30}$ virtual entity. Comparison with the power consumption of physical machines throws the ${ }^{30}$

31

core challenge into sharper relief. With physical machines:

32

33

- the foundational dependence on virtualizing agent is ontologically foreign and 


\begin{tabular}{|c|c|c|c|}
\hline 2 & CAT\# & CODE & PROBLEM \\
\hline 4 & P1 & $\begin{array}{l}\text { Architecture of VEs and PMs - profiling } \\
\text { power consumption }\end{array}$ & $\begin{array}{l}\text { What is the impact of specific major architec- } \\
\text { tural features of computer hardware on the } \\
\text { power consumption of VEs? }\end{array}$ \\
\hline 5 & $\mathrm{P} 2$ & Virtualization genre, platform & $\begin{array}{l}\text { Is it possible to meaningfully rationalize the } \\
\text { behaviour of VE power consumption across }\end{array}$ \\
\hline 6 & & & $\begin{array}{l}\text { different implementations of systems for vir- } \\
\text { tualization? }\end{array}$ \\
\hline 8 & P3 & $\begin{array}{l}\text { Estimation of power consumption of } \\
\text { virtualization-host system }\end{array}$ & $\begin{array}{l}\text { Can host system power consumption be pre- } \\
\text { dicted on the basis of VE activity? }\end{array}$ \\
\hline 9 & $\mathrm{P} 4$ & Workload-adaptable power models & $\begin{array}{l}\text { Can workload-adaptable power models be } \\
\text { developed? }\end{array}$ \\
\hline 11 & P5 & Resource heterogeneity & $\begin{array}{l}\text { How do a VE's power consumption and } \\
\text { power model vary with resource configura- } \\
\text { tion (heterogeneous VEs)? }\end{array}$ \\
\hline 12 & P6 & Influence of temperature on power-model & $\begin{array}{l}\text { How does temperature of operation affect } \\
\text { VEs' power models? }\end{array}$ \\
\hline 13 & P7 & Resource use and measurement in VEs & $\begin{array}{l}\text { How can load be targeted at specific VE re- } \\
\text { sources? How can the actual resource con- } \\
\text { sumption be measured? }\end{array}$ \\
\hline 15 & P8 & Host system power attribution & $\begin{array}{l}\text { How can the measured power consumption } \\
\text { of a host to be attributed to the hosted VEs? }\end{array}$ \\
\hline 17 & P9 & IO Virtualization & $\begin{array}{l}\text { What is the impact of specific major imple- } \\
\text { mentations of IO virtualization on the power } \\
\text { consumption of VEs? }\end{array}$ \\
\hline 19 & P10 & Network functions & $\begin{array}{l}\text { Which particular implementation of a net- } \\
\text { work function is most power or energy effi- } \\
\text { cient? }\end{array}$ \\
\hline 21 & P11 & Software L2 data plane switching & $\begin{array}{l}\text { What is the impact of specific major imple- } \\
\text { mentations of software layer } 2 \text { data plane } \\
\text { switching on the power consumption of VEs? }\end{array}$ \\
\hline 22 & P12 & Investigation of processor green capabilities & $\begin{array}{l}\text { How can we model operation under real-time } \\
\text { exploitation of processor green capabilities? }\end{array}$ \\
\hline 24 & P13 & $\begin{array}{l}\text { Improvement of power efficiency of high- } \\
\text { performance IO virtualization frameworks }\end{array}$ & $\begin{array}{l}\text { How can we improve the power efficiency of } \\
\text { high performance packet IO frameworks? }\end{array}$ \\
\hline 25 & & & \\
\hline & & & \\
\hline
\end{tabular}

- the activity of other physical machines (that do not send or receive workload) $\mathrm{is}_{27}$

29 The seven dimensions of variability are shown in Table 6. It is not surprising that the ${ }^{30}$ problems we have seen researchers tackle are closely aligned with these dimensions. The ${ }^{30}$ ${ }^{31}$ research space is precisely about the need to obtain an understanding of the impact that ${ }^{31}$ ${ }^{32}$ these variables have on power consumption. The scope of models we found in the RUs cov- ${ }^{32}$ ${ }^{33}$ ers only a subspace of the seven-dimensional space but the extent is not usually stated. We ${ }^{33}$ 
Table 4: Approach category codes

\begin{tabular}{|c|c|c|}
\hline 2 & CAT\# & CODE \\
\hline \multirow{2}{*}{3} & A1 & Power attribution principle \\
\hline & A2 & Modeling bias \\
\hline 4 & A3 & Green operating principles \\
\hline \multirow{2}{*}{5} & A4 & Physical analysis \\
\hline & A5 & Identification and use of metrics of energy efficiency \\
\hline 6 & A6 & Managed resource provision (setup time) \\
\hline \multirow{2}{*}{7} & A7 & Controlled resource provision (operation time) \\
\hline & A8 & Resource-specific workloads \\
\hline 8 & A9 & Workloads representative of real use \\
\hline \multirow{2}{*}{9} & A10 & Computing resource instrumentation \\
\hline & $\mathrm{A} 11$ & Workload characterization and instrumentation \\
\hline 10 & $\mathrm{~A} 12$ & Direct power measurement \\
\hline \multirow{2}{*}{11} & $\mathrm{~A} 13$ & Hardware instrumentation of predictors \\
\hline & A14 & Simulation \\
\hline 12 & A15 & Use test data in post-training phase \\
\hline \multirow{2}{*}{13} & A16 & Corroboration through experimentation \\
\hline & A17 & Model adaptation technique \\
\hline
\end{tabular}

14

${ }^{32}$ touch upon this issue briefly in section 4.3 .3 (research without context) and section 4.4.1 ${ }^{32}$

${ }^{33}$ (universal power model). 


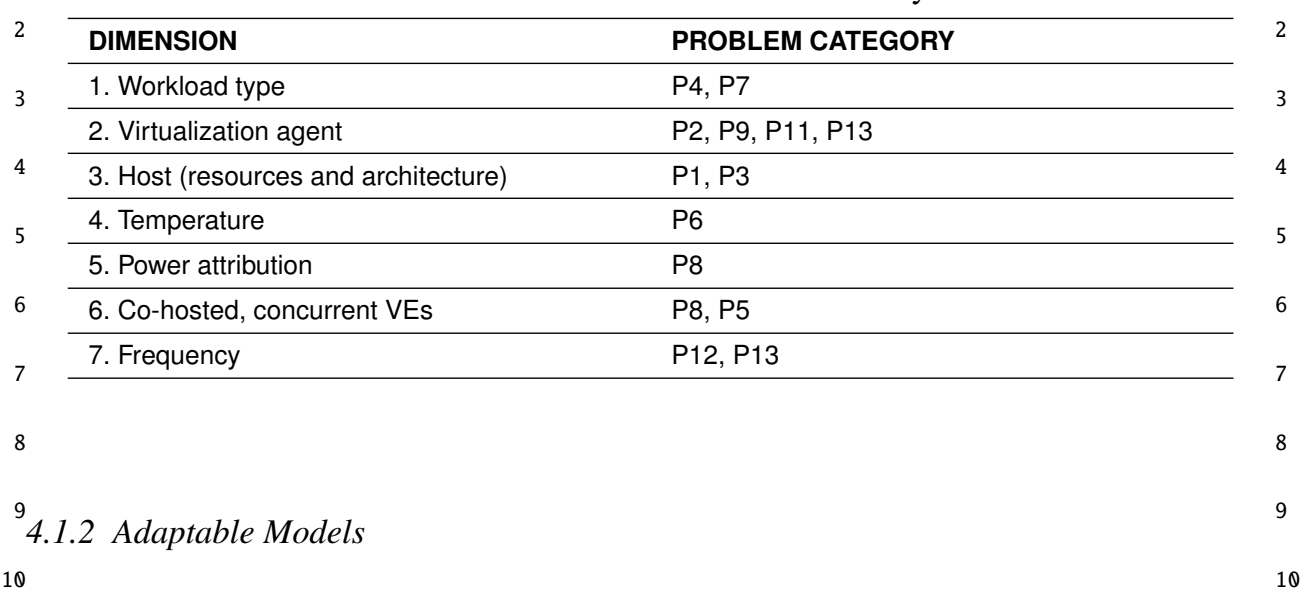

We observe three conditions which must be met for an automated modeling system to ${ }_{11}$ ${ }_{12}$ obtain a model of VE power consumption. The first, fundamental condition is common to ${ }_{12}$ ${ }_{13}$ all successful research into modeling of VE power consumption.

141 Resources utilized by the VE (whether measured through architectural or microar-14 15 chitectural instrumentation) must be strongly correlated to power consumption by 15 16 the VE as well as power consumption in host system overheads. If the overheads16 17 are uncorrelated to the VE's activity, or weakly so, then any significant power over-17 18 head must be modelled through separate consideration of its driving causes, e.g.18 19 temperature [93]. Furthermore, in order that modeling may apply across a diversity 19 20 of operating contexts, two other conditions must hold.

212 Any change in the parameters of correlation must be dynamically learnt and the 21

233 Any change in operating context that invalidates parameters of correlation, must be 23 of finite duration. An indefinite transient precludes the formation of a model. $\quad 24$

25 Two of the seven dimensions are commonly investigated in evaluation of modeling sys- ${ }^{25}$ ${ }^{26}$ tems: workload type and co-hosted, concurrent VEs. We suggest that a modeling system ${ }^{26}$ ${ }^{27}$ may be labelled adaptable if, minimally, it meets the above three conditions under these ${ }^{27}$ ${ }^{28}$ two dimensions, i.e. (a) operations of variable workload type per VE, and (b) a variable ${ }^{28}$ ${ }^{29}$ number of concurrent, co-hosted VEs. Before proceeding to refer to evaluations observed ${ }^{29}$ ${ }^{30}$ in the RUs, it is useful to draw attention here to the need for a modeling system, rather ${ }^{30}$ ${ }^{31}$ than simply a model, in the estimation of power consumption. Dynamic adjustments are ${ }^{31}$ ${ }^{32}$ effected through the intervention of such a system, which adjusts model parameters to the ${ }^{32}$ 33 operating context. 
${ }^{1}$ Workload type Different types of workload correspond to different use of resources; ${ }^{1}$

${ }^{2}$ hence, behavior of power consumption also differs. Several researchers $[63,87,88]$ stud- $^{2}$

${ }^{3}$ ied the effect of changing workload type either by categorizing the workload itself or by ${ }^{3}$

${ }^{4}$ categorizing the resources consumed by a specific kind of workload. Research studies [87] ${ }^{4}$

${ }^{5}$ and [88] characterized the use of CPU utilization for workloads, which is known to have ${ }^{5}$

${ }^{6}$ a workload-type dependent relationship with power consumption [75]. Thus, in both [87] ${ }^{6}$

${ }^{7}$ and [88], the need arises to re-train on change of workload type (second condition). Also, ${ }^{7}$

${ }^{8}$ note the observation in both [87] and [88] about the duration of re-training for refined mod- $^{8}$

${ }^{9}$ els (third condition). In [63], energy consumption for multimedia applications is studied, ${ }^{9}$

${ }^{10}$ which identifies the use of forwarding engines for different data-planes as one major cause ${ }^{10}$

${ }^{11}$ of increase in power consumption. 11

12 On the other hand, each one of $[75,82,91,93,94]$ is capable of adapting to different ${ }^{12}$

${ }^{13}$ workload types without re-training. All predictors here are event counters. However, events ${ }^{13}$

${ }^{14}$ are not necessarily linear predictors of power consumption across all workload types. $\mathrm{In}^{14}$

${ }^{15}$ [96], it was found that the model parameters of a linear regression of event counters onto ${ }^{15}$

${ }^{16}$ power consumption are workload specific. Given the contrast with the other works cited ${ }^{16}$

${ }^{17}$ above, it seems that the root cause is the selection of events for prediction. $\quad{ }^{17}$

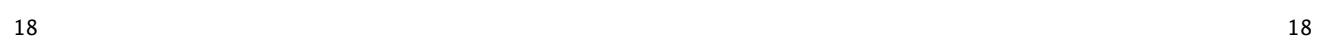

${ }_{19}$ Concurrent operation In [74], a non-linear model of the dynamic power of a multi-19 ${ }_{20}$ (virtual)-core VM is obtained. The dynamic power $p_{v m}$ is expressed in terms of the average ${ }_{20}$ ${ }_{21}$ utilization $\left(u_{v}\right)$ which $n$ virtual cores impose on a total of $N$ physical cores: 21

${ }^{24}$ Parameters $\alpha$ and $\beta$ are determined through linear regression of the logarithmic form. The ${ }^{24}$ ${ }^{25}$ relationship was successfully tested under the operating context of one VM and three VMs. ${ }^{25}$

${ }^{26}$ The model is limited to processor-intensive workloads; indeed, this is not surprising, since ${ }^{26}$ ${ }^{27}$ the predictor is (virtual) processor utilization. However, a conclusion can be drawn: the ${ }^{27}$ ${ }^{28}$ accuracy of the model in predicting the dynamic power of an individual VM is independent ${ }^{28}$

29 f concurrent operation of other VMs (albeit for a limited range of workload types). $\quad 29$ of concurrent operation of other VMs (albeit for a limited range of workload types).

30 In [87], it is shown that estimation based on processor utilization can accurately predict ${ }^{30}$ ${ }^{31}$ the power consumption of multiple concurrent VEs. The prerequisite is that a model for that ${ }^{31}$

${ }^{32}$ VE's workload type has been learnt. The conclusion to be drawn here is the same as that ${ }^{32}$ ${ }^{33}$ drawn for [74]: that the prediction accuracy for individual VEs is independent of concurrent ${ }^{33}$ 
${ }^{1}$ operation. Furthermore, the range of workload types has expanded to a broader range. With ${ }^{1}$

${ }^{2}$ regard to results obtained in [96], the conclusion is yet again the same: individual prediction ${ }^{2}$

${ }^{3}$ accuracy (albeit workload-type-dependent) is independent of concurrent operation. ${ }^{3}$

4 Independence of workload type is manifestly claimed in [75, 82, 91, 93]. Furthermore, ${ }^{4}$

${ }^{5}$ the first three of these have been evaluated under multiple concurrent VEs. It is noteworthy, ${ }^{5}$

${ }^{6}$ therefore, that not only is the modeling system adaptable, but it also produces a model that ${ }^{6}$

${ }^{7}$ is itself adaptable without the need for real-time adjustment. In [91], evaluation is restricted ${ }^{7}$

${ }^{8}$ to a single VE; therefore, this modeling system does not meet our conditions of adaptability. ${ }^{8}$

9 We therefore conclude that limited adaptability (defined as independence of workload ${ }^{9}$

${ }^{10}$ type and number of concurrent, co-hosted VEs) has been achieved. From surveyed RUs, ${ }^{10}$

${ }^{11}$ we conclude that presently, the limits of adaptability are the following. 11

121 Workload type: Power consumption of processor-intensive, memory-intensive, disk- ${ }^{12}$

13 intensive workloads and mixes thereof, has been modeled by a single modeling sys- ${ }^{13}$

14 tem in an automated manner:- 14 tem in an automated manner.

152 Co-hosted, concurrent VEs have been modeled up to but not exceeding over- ${ }^{15}$

$16 \quad 16$ commitment of processor physical cores.

17 Caution has been exercised in claiming these limits. For example, while commitment ${ }^{17}$

18 Caf 18

of logical cores (e.g. Intel Hyper-Threading logical cores) has been investigated, we have

${ }^{19}$ found no evidence that power consumption has been modeled in a manner that automati- ${ }^{19}$

20

cally adapts to a transition of consumption from physical to logical cores.

21

${ }^{22} 4.1 .3$ Lack of use of metrics of energy efficiency and standards to address this deficiency ${ }^{22}$

${ }^{23}$ We note several experiments, e.g. $[59,60,81,83,114-116]$ that target power consump- ${ }^{23}$

${ }^{24}$ tion but less than $12 \%$ of the RUs in our corpus approach the problem in terms of some ${ }^{24}$

${ }^{25}$ energy efficiency metric [57, 58, 78, 97]. It is necessary to move beyond measurements ${ }^{25}$

${ }^{26}$ of how much power was consumed, to measurements of how much power was consumed ${ }^{26}$

${ }^{27}$ to carry out a specific task. This change in approach facilitates comparison between re- ${ }^{27}$

${ }^{28}$ search works. More importantly, it directly addresses the question about cost of operation ${ }^{28}$

$\begin{array}{ll}29 & \\ & \text { of infrastructure. }\end{array}$

30 This approach requires identification of a unit of comparison that transcends the bound- ${ }^{30}$

${ }^{31}$ aries of disparate systems that deliver this unit. This unit of comparison is referred to in ${ }^{31}$

${ }^{32}$ the Life Cycle Assessment (LCA) framework (ISO 14040) [117] as the functional unit. ${ }^{32}$

${ }^{33}$ A definition specific to telecommunications equipment is given in [118] : the functional ${ }^{33}$ 
${ }^{1}$ unit is defined as "a performance representation of the system under analysis". Since this ${ }^{1}$

${ }^{2}$ definition is too broad to serve as a standard, units specific to a variety of classes of equip- ${ }^{2}$

${ }^{3}$ ment are defined too [118]. In our corpus, two approaches we have seen are hash/J [57] and $^{3}$

${ }^{4} \mathrm{~J} /$ Web Interaction [78]. The functional units in these cases are performance of one crypto- ${ }^{4}$

${ }^{5}$ hash and one web interaction, respectively. Another is to define a functional unit specific ${ }^{5}$

${ }^{6}$ to a digital service delivered over a telecommunications network, e.g. ten minutes' time of ${ }^{6}$

${ }^{7}$ browsing [119]. Note that L.1310 [[118], p.4] recognizes both metrics where energy is in ${ }^{7}$

${ }^{8}$ the numerator [78] as well as those where it is in the denominator [57]. Guidance is avail- ${ }^{8}$

${ }^{9}$ able: energy efficiency measurement for several NFV components has been standardized, ${ }^{9}$

${ }^{10}$ as well as measurement standards for servers, switches and virtualization systems [120]. ${ }^{10}$

11

${ }^{12} 4.1 .4$ Trends 12

${ }^{13}$ Here, we present trends which we have identified through our collation of problems, ap- ${ }^{13}$

${ }^{14}$ proaches and developments. This sub-sub-section is divided into four parts: section $4.1 .4^{14}$

${ }^{15}$ regards trends in tackled problems; section 4.1.4 in complexity of tackled problems; sec- ${ }^{15}$

${ }^{16}$ tion 4.1.4 in approaches and section 4.1.4 in developments. Within each part, trends are ${ }^{16}$

${ }^{17}$ numbered using Arabic numerals, to differentiate cleanly between them. 17

$18 \quad 18$

${ }^{19}$ Challenges $\quad{ }^{19}$

201 The causality DAG (Fig. 7) shows that the research space can be characterized suc- ${ }^{20}$

21 cinctly: note the thickness of the links originating at P7 (VE resource use and mea 21

2222

23 (a) The graphic in Fig. 1 and the bar chart in Fig. 9 show that the challenge- ${ }^{23}$ category tackled most frequently $\left(R_{P_{7}}=25.3 \%\right)$ is how to load the VE's re- ${ }^{24}$ sources and measure the loading (P7). P7 also has the most frequent presence ${ }^{25}$ in RUs: $F_{P_{7}}=65.7 \%$ (see Fig. 9). 26

(b) The accurate specification and measurement of load is essential to model for- ${ }^{27}$ mation. These measurements provide the aggregated (i.e. indiscriminate of ${ }^{28}$ which VE is consuming) predictors - the input side of the model. Disaggregat- ${ }^{29}$ ing the predictors and attributing measured power consumption to the individ- ${ }^{30}$ ual VEs (the output side of the model) constitutes the second most frequently- ${ }^{31}$ tackled challenge $\left(R_{P_{8}}=16.5 \%\right)$. P8 also has the second most frequent pres- ${ }^{32}$ ence in RUs: $F_{P_{8}}=42.9 \%$. 
(c) In the following, the vector (P,A,D,weight) will be used to indicate a path $^{1}$ $(\mathrm{P}, \mathrm{A}, \mathrm{D})$ and the number of triads (weight) through the path.

i. $34.2 \%$ of triads collected regard P7 and P8 respectively.

ii. The triads graphic (Fig. 8) shows that efforts rooted in these two chal- ${ }^{4}$ lenges converge on a common objective: building adaptable models of ${ }^{5}$ VEs' power consumption, notably using regressions to linear combina- ${ }^{6}$ tions of computing resource predictors.

iii. The set of triads leading to linear regressions consists of $(7,10,5,20),{ }^{8}$ $(8,17,5,13),(7,9,5,12),(8,1,5,10),(8,2,5,8),(7,8,5,6),(8,12,5,4),(7,6,5,3)^{9}$ and these account for $11.3 \%$.

2 The third most frequently-tackled challenge is that of estimation of virtualization- ${ }^{11}$ host-system power consumption $\left(R_{P_{3}}=15.4 \%\right)$. This category might be overlooked ${ }^{12}$ in a first inspection of the research space, as it might seem futile to attempt to estimate ${ }^{13}$ a power consumption which can be measured. However: in practice, the logistical ${ }^{14}$ challenge of measurement of horizontally-scaled system deployments seems to be ${ }^{15}$ well known and several works have been undertaken to develop software meters. 16

3 Several significant links originate on P1 (profiling power consumption's dependency ${ }^{17}$ on architecture). The DAG (Fig. 7) indicates that approaches to tackling P1 are ${ }^{18}$ spread across a mixture of managing resource provision, use of synthetic (resource- ${ }^{19}$ specific) workloads and prediction using system software's instrumentation. 20

4 Another large group of links originates on P3 (estimation of host system power con- ${ }^{21}$ sumption). The DAG (Fig. 7) indicates that a primary concern in tackling P3 is ${ }^{22}$ the type of model to select. The most common choices are linear and non-linear; ${ }^{23}$ machine-learning techniques are the least common of the three. The thickness of the ${ }^{24}$ triad $(3,2,10,11)$ (D10: relative accuracy of formal approaches), indicates that there ${ }^{25}$ is already significant interest in whether the advanced models are worth the effort to ${ }^{26}$ develop them and computational resources required to operate them. 27

5 While $F_{P_{7}}=65.7 \%$ (loading VEs and measuring their use of resources), only $8.6 \%{ }^{28}$ $\left(F_{P_{7.2}}=8.6 \%\right)$ of all RUs investigate loading containers and measuring their use of ${ }^{29}$ resources. With virtual dataplane devices, the figure is even lower: $F_{P_{7.3}}=2.9 \%{ }^{30}$ This imbalance suggests that there is much room left for research into modeling ${ }^{31}$ power consumption by containers and dataplane devices. 
${ }^{1}$ Challenge complexity In [45, section 7.3], we suggest that diversity of approaches is a ${ }^{1}$ ${ }^{2}$ metric of the complexity of a challenge. We note (see Fig. 9) that challenge complexity ${ }^{2}$ ${ }^{3}\left(W_{P_{k}}\right)$ generally follows the frequency with which a particular challenge-category is tack- ${ }^{3}$

${ }^{4}$ led. That is: the more frequently the challenge is addressed, the more diverse are the ap- ${ }^{4}$ ${ }^{5}$ proaches applied to it. This can be verified by noting that the heights of both sets of bars in ${ }^{5}$ ${ }^{6}$ the chart (superimposed on the same graphic) follow roughly the same pattern. However, ${ }^{6}$ ${ }^{7}$ some categories do stand out. For example: ${ }^{7}$

8

${ }_{22}$ Approaches Our approach utility metric, $U_{A_{k}}$ (see [45, section 7.5] and Fig. 10), seems to22 23be a useful one. It communicates clearly what we have observed in our surveying. Below,23 24we draw attention to saliencies perceived during surveying and confirmed by the metrics. 24 challenge. Model bias might be attributed to this challenge too but largely, model bias is determined by other challenges within the scope of the RU.

2 While host system power attribution (P8) has the second highest research interest (and frequency of occurrence, $F_{P_{k}}$ ), the number of approaches taken to solve this challenge is relatively small.

3 On the other hand, power consumption's dependencies are tackled by a disproportionately large number of approaches. This is not surprising, as the objects of study ${ }_{17}$ (architecture, virtualization platform, virtualization genre) are multi-faceted and dependencies can be investigated through a variety of approaches.

1 Instrumentation of consumption of computing resources (A10 - which includes mi- ${ }^{25}$ croarchitectural instrumentation and that abstracted by system software) is repeat- ${ }^{26}$ edly adopted $\left(U_{A_{10}}=15.5 \%, R_{A_{10}}=15.7 \%\right)$ in empirical work in this field. It is ${ }^{27}$ also the most utilized of all approaches. In comparison, workload instrumentation ${ }^{28}$ accounts for $8.2 \%$ utilization $\left(R_{A_{11}}=5.2 \%\right)$.

2 Resource-specific workloads (A8) are the more utilized approach to loading VEs ${ }^{30}$ (14.3\% of all triads). This approach category is the second most utilized. Workloads ${ }^{31}$ representative of real use (A9) account for $9.0 \%$ of all triads. The corresponding ${ }^{32}$

1 P6 regards the influence of temperature on the power model. Approach diversity is poor here because only the use of additional instrumentation can be attributed to this frequency of occurrence figures $\left(R_{A_{k}}\right)$ are $13.4 \%$ and $10.0 \%$ respectively. 
13 Two other high-utility approaches are (a) modeling bias (A2, with $U_{A_{2}}=11.9 \%, R_{A_{2}} \stackrel{1}{=}$ $11.6 \%$ ) and (b) the managed provision of resources (A6, with $U_{A_{6}}=10.2 \%, R_{A_{6}}={ }^{2}$ $10.8 \%$ ). Use of representative workloads (A9) follows at $U_{A_{9}}=9.0 \%, R_{A_{9}}=10.1 \%$. $^{3}$

4 A comparison of the patterns of bar height distribution for $U_{A_{k}}$ and $R_{A_{k}}$ reveals that ${ }^{4}$ some categories stand out.

(a) While A12 (direct power measurement) is not as frequent as the other software- ${ }^{6}$ based forms of instrumentation, this approach has a utility that sticks out of the ${ }^{7}$ pattern (see Fig. 10). The reason is that most, if not all, developments obtained ${ }^{8}$ in an RU that includes this approach depend on the directly measured power. 9

(b) Similarly, while workload characterization and instrumentation (A11) is em- ${ }^{10}$ ployed with a frequency that is about one-third that of its alternative (i.e. com- ${ }^{11}$ puting resource instrumentation, A10), it has a far better utility-to-frequency ${ }^{12}$ ratio than A10. The triads graphic (Fig. 8) indicates that one important cause of ${ }^{13}$ this high utility is that software-L2-data-plane switches and (virtual) network ${ }^{14}$ functions are investigated primarily using measurement of workload (and not ${ }^{15}$ measurement of computing resources consumed to process a workload). $\quad 16$

$155.6 \%$ of all identified developments are obtained in modelling power consumption. ${ }^{19}$ The remaining $44.4 \%$ regard how power consumption depends on implementations. Implementations investigated (for their impact on power consumption) range from ${ }^{21}$ entire virtualization platforms (e.g. KVM) to components (e.g. processors). 22

2 The most frequent $\left(R_{D_{12}}=14.5 \%\right)$ category of developments is that which regards ${ }^{23}$ observations on dependency of power consumption on architecture. The cause of this ${ }^{24}$ high frequency is that developments in the study of architecture establish directional ${ }^{25}$ (negative, positive, neutral) correlations rather than predictive forms. For example, ${ }^{26}$ in a single RU [80], all of the following developments emerge:

(a) D12.08: VM power consumption increases linearly with vCPU frequency of ${ }^{28}$ operation when the vCPU is operating at $100 \%$ utilization;

(b) D12.09: Virtualization-host-system power consumption increases linearly with ${ }^{30}$ the number of physical cores operating at $100 \%$ utilization;

(c) D12.1: Virtualization-host-system power consumption increases with the num- ${ }^{32}$ ber of VMs active on the same core 
13 Amongst developments of models, the most common category (D8) is of the type ${ }^{1}$

2 where power consumption (host or VE) is predicted in terms of workload character- ${ }^{2}$

${ }^{24} 4.1 .5$ Three levels of abstraction

${ }^{25}$ We note that existing power models may be classified into one of three levels of abstraction. ${ }^{25}$

${ }^{26}$ In ascending order of abstraction, these are:

$27 \quad 1$ Microarchitecture and architecture

282 Simple characterization of workload

293 Complex characterization of workload

30

31 referring to the variables used as model inputs. In all cases, the variables are some measure

${ }^{32}$ of load. At the lowest level, inputs that quantify operation of processor sub-units are used ${ }^{32}$

${ }^{33}$ (event counters and event timers). The highest level uses inputs that quantify the demand ${ }^{33}$ 
${ }^{1}$ for a telecommunications system or service. Clearly, the latter inputs are far more detached ${ }^{1}$

${ }^{2}$ from the underlying, concrete implementation than the former. ${ }^{2}$

3 Many power models use hardware resource consumption to estimate the power consumed ${ }^{3}$

${ }^{4}$ by virtual components. A study was conducted in [116], to evaluate the available power ${ }^{4}$

${ }^{5}$ models for processors, VMs and servers. According to this survey, most of the power mod- ${ }^{5}$

${ }^{6}$ els for virtual machines use physical machine counters to estimate the corresponding re- $^{6}$

${ }^{7}$ source utilization by the virtual components. We offer further insight on this matter. $\mathrm{We}^{7}$

${ }^{8}$ concur in the observation that much current research is concerned with modeling power ${ }^{8}$

${ }^{9}$ consumption of virtual machines. The approach may succinctly be described as estimates ${ }^{9}$

${ }^{10}$ obtained from models trained out of either architectural or microarchitectural instrumen- ${ }^{10}$

${ }^{11}$ tation data. We distinguish between architecture and microarchitecture using the classical ${ }^{11}$

${ }^{12}$ interpretation [[121], p.17]. Now, the term "architecture" is severely overloaded and its in ${ }^{12}$

${ }^{13}$ terpretation can easily differ from that which we wish to exploit. In the following descrip- ${ }^{13}$

${ }^{14}$ tion of the levels of abstraction, we use the alternative "system software's instrumentation"14

${ }^{15}$ to convey the same meaning as "architectural instrumentation" with less ambiguity. 15

16 Microarchitectural instrumentation: This is the lowest level of instrumentation. Power ${ }^{16}$

${ }^{17}$ consumption is expressed in terms of variables that are defined at sub-CPU and sub- ${ }^{17}$

${ }^{18}$ subsystem levels. The granularity of this level holds the greatest potential for accuracy, but ${ }^{18}$

${ }^{19}$ the rate of change of observed variables has deterred several researchers from pursuing this ${ }^{19}$

${ }^{20}$ approach to instrumentation, citing concerns about communicational and computational ${ }^{20}$

${ }^{21}$ overhead. This concern has been dismissed by three groups of researchers [52, 98, 109], ${ }^{21}$

${ }^{22}$ who have indicated that acceptable accuracy can be achieved with negligible overheads. ${ }^{22}$

${ }^{23}$ Given the proliferation of works based on this approach, the availability of high-level lan- ${ }^{23}$

${ }^{24}$ guage code that facilitates use and the potential for capture of physical behaviors, then our ${ }^{24}$

${ }^{25}$ general recommendation is a preference to investigate use of microarchitecture instrumen- ${ }^{25}$

${ }^{26}$ tation. $\quad 26$

27 System software's instrumentation: This regards a class of instrumentation that has mean- ${ }^{27}$

${ }^{28}$ ing across the spectrum of computer systems. The input variables, such as CPU or network ${ }^{28}$

${ }^{29}$ interface utilization, are produced by some digest (function) of intermediary system soft- ${ }^{29}$

${ }^{30}$ ware. For example, an operating system's (OS) measurement of core utilization can roughly ${ }^{30}$

${ }^{31}$ be described as the core's duty cycle on behalf of the OS. This is significantly removed from ${ }^{31}$

${ }^{32}$ knowledge of activity within the core. While models at this level are more abstract, they ${ }^{32}$

33 are still low-level, especially when compared with the other levels. 33 are still low-level, especially when compared with the other levels. 
1 A less frequently encountered abstraction is used by Enokido, Takizawa and various oth- ${ }^{1}$

${ }^{2}$ ers with whom they have co-published. They use simple characterization of workload e.g. ${ }^{2}$

${ }^{3}[81,83,122]$. These models describe power consumption in terms of fundamental de- ${ }^{3}$

${ }^{4}$ scriptors of workload, e.g. number of processes and transmit/receive data rate. The least ${ }^{4}$

${ }^{5}$ granular model uses a complex characterization of workload [31, 123]. The objective here ${ }^{5}$

${ }^{6}$ is to quickly proceed to a good estimate of the power or energy required to produce the ${ }^{6}$

${ }^{7}$ workload. This kind of model has no use in real-time control but it is useful for macro- ${ }^{7}$

${ }^{8}$ scopic comparisons, i.e. comparisons between two disparate systems for provision of $a^{8}$

${ }^{9}$ service. Possibly, the disparity is paradigmatic; for example: classical vs virtualized im- ${ }^{9}$

${ }^{10}$ plementations. Thus in [31], the implied (system) metric is the amount of power required ${ }^{10}$

${ }^{11}$ to deliver 1 million packets per second of throughput through an evolved packet-core's ${ }^{11}$

${ }^{12}$ (EPC) serving gateway (SGW). In [123-125] the objective is to minimize the amount of ${ }^{12}$

${ }^{13}$ consumed power by virtualizing baseband processing functions, evolved packet core, cus- ${ }^{13}$

${ }^{14}$ tomer premises equipment, and radio access network functions. 14 15

164.1.6 Service determinism: a criterion particular to the telco cloud 16

${ }^{17}$ The NFV data plane demands determinism [107, 126], [65] @ 25:45 ${ }^{[2]}$. Strictly, service ${ }^{17}$

${ }^{18}$ determinism is sought, since the packet arrival process is generally unconstrained. The $\operatorname{root}^{18}$

${ }^{19}$ cause of this need is to correctly size equipment resources to meet load demands, whether ${ }^{19}$

${ }^{20}$ throughput, latency or jitter. This need is intense, as it impinges on a PTNO's obligations, ${ }^{20}$

${ }^{21}$ specified in legally-binding service-level agreements (SLAs). 21

22 Service determinism has been approached through augmentation of GP hardware, with ${ }^{22}$

${ }^{23}$ domain-specific architectures (DSAs, which we first referred to in 3.1.1). Hardware-centric ${ }^{23}$

${ }^{24}$ DSAs for the NFV data plane are constructed out of elements (or systems thereof) that can ${ }^{24}$

${ }^{25}$ be divided into three groups. 25

261 IO system architecture: This group comprises the abstraction, directly at a periph ${ }^{26}$

27 eral interface, of functionality that facilitates virtualization of hardware, e.g. SR- ${ }^{27}$

28 IOV (used in PCI - Peripheral Component Interconnect) and N-port ID virtualization ${ }^{28}$

$29 \quad$ (Fibre Channel). 29 (Fibre Channel).

302 Processor architecture: This comprises architectural change that facilitates parti- ${ }^{30}$

31 tioning of processor resources, such as multi-core processors and NUMA. 31

${ }^{32}$ We use the @ symbol since a video is being cited and the content cited here may be found ${ }^{32}$

${ }^{33}$ by seeking the time indicated. 
13 Co-processing: Compression and decompression, encryption and decryption, and ${ }^{1}$

2 packet header processing are examples of high-volume tasks that can be offloaded to ${ }^{2}$

3 co-processing subsystems. Examples include Intel QuickAssist Technology (QAT) ${ }^{3}$

4 and TCP Offload Engine (TOE).

5 We further observe a set of approaches that are complementary to DSAs in the quest for ${ }^{5}$

${ }^{6}$ determinism. These include:

6

1 Large memory pages: exploitation of the facility to organize virtual memory into ${ }^{7}$ larger pages than the GP $4 \mathrm{KiB}$;

92 Userspace programming: diversion of control of hardware resources away from the ${ }^{9}$ multi-service kernel, to single-common-use user space programs, e.g. DPDK and ${ }^{10}$

11 AF_XDP.

${ }^{12}$ Notably, resource partitioning (second hardware-centric approach), combined with userspace

${ }^{13}$ programming (second complementary approach), realize the run-to-completion scheduling ${ }^{13}$ 14 model [107].

15 The cost of current realizations of service determinism. The (aforementioned) combi- ${ }^{15}$

16 nation of core partitioning and userspace programming has been widely adopted through 16

${ }^{17}$ Intel's popularization of DPDK, via Intel's open source liaison efforts. The multi-core pro- 17

Intel's popularization of DPDK, via Intel's open source liaison efforts. The multi-core pro-

${ }^{18}$ cessor enables service determinism through an approach that is anathema to the principles ${ }^{18}$

19

of multiprogramming: dedication of hardware to a specific task.

20

${ }^{21}$ 4.1.7 Direct or indirect measurement of power consumption in virtualized environments? ${ }^{21}$

${ }^{22}$ We observe that most research in modeling power consumption seeks to obviate the need ${ }^{22}$

${ }^{23}$ for direct measurement through indirect measurement, namely: of resource use which has ${ }^{23}$

24 discoverable relationship with power consumption by the entity hosting the resources. 24 relationship with power consumption by the entity hosting the resources.

${ }^{25}$ Modeling, here, has the objective of indirect measurement of a variable that is not directly ${ }^{25}$ ${ }^{26}$ accessible (power consumption by VEs), through others which have convenient and re- ${ }^{26}$

${ }^{27}$ liable instrumentation. The accessible variables are referred to as (power) proxies. The ${ }^{27}$

${ }^{28}$ RAPL interface provides a unique approach to measurement as it directly addresses power ${ }^{28}$

${ }^{29}$ consumption. However, notwithstanding appearances of direct measurement, RAPL is ac- ${ }^{29}$

${ }^{30}$ tually based on a software model that uses performance-monitoring counters (PMCs) as ${ }^{30}$

${ }^{31}$ predictor variables to measure power consumption. It is available in processors starting ${ }^{31}$

${ }^{32}$ from the Sandy Bridge microarchitecture. RAPL measures the power consumption of dif- ${ }^{32}$

${ }^{33}$ ferent physical domains, where each domain consists of either cores, sockets, caches, or ${ }^{33}$ 
${ }^{1} \mathrm{GPU}$. We briefly denote its accuracy through references to research that has investigated ${ }^{1}$ ${ }^{2}$ them.

41 A study was conducted to find the advantages and drawbacks of using RAPL [109]. ${ }^{4}$ Different Intel's architectures such as Sandy Bridge, Haswell and Skylake were used ${ }^{5}$ in the experiments to analyze RAPL's accuracy and overhead. Data collected were ${ }^{6}$ modeled using a linear model and a Generalized Additive Model (GAM). Accuracy ${ }^{7}$ of predicted results was compared with the measured power consumption from $\mathrm{a}^{8}$ precise external hardware power meter where RAPL based models show $1.8-4.3 \%$ of ${ }^{9}$ error for the various architectures. Prediction accuracy of RAPL-based power models ${ }^{10}$ was also compared with those based on OS counters, where OS-based models show ${ }^{11}$ a higher error of $5-16 \%$. Also, the performance overhead (in terms of time) of using ${ }^{12}$ RAPL was studied at different sampling frequencies and for different application ${ }^{13}$ runs. Results show that even with high sampling frequency of $1100 \mathrm{~Hz}$, RAPL incurs ${ }^{14}$ support to read energy counters, overflow of registers due to their 32-bit size and ${ }^{16}$ measurement of energy consumed by individual cores.

2 Another study to analyze the precision of RAPL is presented in [127], where only ${ }^{18}$ the dynamic change in power consumption is observed. WattsUp Pro, an external ${ }^{19}$ power measurement unit, is used as a reference for power measurement values. Intel ${ }^{2 \theta}$ Haswell and Skylake servers were used in the experiments to run different appli- ${ }^{21}$ cations and to find the reliability of RAPL with the help of external power meters. ${ }^{22}$ However, in this research work, only two power domain packages (power consump- ${ }^{23}$ tion of whole socket and DRAM domain of RAPL) were observed. Applications ${ }^{24}$ such as dense matrix multiplication and 2D Fast Fourier Transform were used for ${ }^{25}$ server power profiling. Results show that the power measurement error varies with ${ }^{26}$ changing application and its workload size. For different applications the average ${ }^{27}$ measurement error using RAPL was in the range of $13-73 \%$ considering WattsUp ${ }^{28}$ power meter as the ground truth. It was concluded that with the modern multi-core ${ }^{29}$ parallel processing and resource contention for shared resources, there is a complex ${ }^{30}$ non-linear relation between performance, workload size and energy consumption. ${ }^{31}$ Hence it is difficult to attain low error percentage for power measurement using on- ${ }^{32}$ chip sensors. 
${ }^{1} 4.2$ Research gaps

${ }^{2}$ With interest focused on the telco cloud, three significant challenges remain unaddressed, ${ }^{2}$

${ }^{3}$ while a fourth requires further attention:

41 modeling of containers' power consumption;

52 the effect of overcommitment on power efficiency; 5

$6 \quad 3$ investigation and classification of DPDK applications and 6

74 the fourth challenge, which is starting to receive some attention (as seen in [64, 95, ${ }^{7}$ $97,105])$, regards modeling of power consumption by virtualized I/O.

Note that our treatment of research gaps does not address improvements in approaches. ${ }^{9}$

${ }^{10}$ We have already indicated, in section 4.1.4, that more adaptable modeling methods are ${ }^{10}$

${ }^{11}$ required to tackle the dimensionality of the field (see section 4.1.1). Indeed, a treatment of ${ }^{11}$

${ }^{12}$ adaptable models is undertaken in section 7.2.2. Similarly, we do not include lack of use of ${ }^{12}$

${ }^{13}$ metrics of energy efficiency (section 4.1.3) with research gaps, as it is a deficiency in the ${ }^{13}$

14 approaches, not a challenge in itself. Rather, here, our attention focuses on where the more 14

15

$\begin{array}{ll}\text { pressing challenges lie for development of the telco cloud. } & 16\end{array}$

${ }^{17}$ Modeling of containers' power consumption Few works [90, 93, 94] tackle containers ${ }^{17}$

${ }^{18}$ from the perspective of their power consumption. Yet VMs are no longer the destination for ${ }^{18}$

${ }^{19}$ the telco cloud (see, for example,[37] and [128]@ 10:30). Containers have replaced virtual ${ }^{19}$

${ }^{20}$ machines as the base for deployment of virtual network functions. In [90], the approach(- ${ }^{20}$

${ }^{21}$ set) taken is to: $\quad 21$

${ }^{22} 1$ use representative workloads e.g. HEVC (High Efficiency Video Coding) transcod- ${ }^{22}$

$23 \quad$ ing and machine learning image classification, amd 23

242 customization of the set of low-level instruments used to correlate power and energy ${ }^{24}$

25 consumption with workload characteristic. 25

${ }^{26}$ This work develops a profiling tool: it provides guidance that is specific to application and ${ }^{26}$

${ }^{27}$ both the hardware and software aspects of the containing platform. "[P]olicy" for "tradeoff ${ }^{27}$

${ }^{28}$ between energy, power and application performance" is the cited objective. Given the high- ${ }^{28}$

${ }^{29}$ dimensionality of the core challenge, this approach-set to modelling and measurement of ${ }^{29}$

${ }^{30}$ power consumption may well be more coherent with the European Telecommunications ${ }^{30}$

${ }^{31}$ Standards Institute's (ETSI) Management and Orchestration (MANO) standard. Such in- ${ }^{31}$

${ }^{32}$ formation would then be included in the infrastructure-resource-requirements meta-data ${ }^{32}$

33 33

descriptors in the VNF package [129]. 
${ }^{1}$ The effect of overcommitment on power efficiency Overcommitment consists of the allo- ${ }^{1}$

${ }^{2}$ cation of more capacity of some compute resource to VEs, than is physically installed. The ${ }^{2}$

${ }^{3}$ concept is very similar to oversubscription of telecommunications capacity to subscribers, ${ }^{3}$

${ }^{4}$ such as when the arithmetic sum of capacities of access links exceeds the aggregating ${ }^{4}$

${ }^{5}$ device's backhauling capacity to a central office/local exchange. In this context, overcom- ${ }^{5}$

${ }^{6}$ mitment principally concerns processing cycles and memory space. ${ }^{6}$

7 As with oversubscription, there is an optimization problem to solve. One problem of in- ${ }^{7}$

${ }^{8}$ terest to this survey's scope is understanding the relationship (say, ratio) of committed vir- ${ }^{8}$

${ }^{9}$ tual resources to installed physical resources that optimizes total cost of ownership (TCO) ${ }^{9}$

${ }^{10}$ of cloud infrastructure: 10

11 - on the one hand, the facility to overcommit has a direct impact on the density of ${ }^{11}$

12 packing of VEs (number of concurrently active VEs) on a virtualization-host-system, ${ }^{12}$

13 thereby reducing the TCO. 13

${ }^{14}$ on the other hand, overcommitment may reduce the power efficiency of a workload. ${ }^{14}$

15 Had this challenge been tackled in any depth, or at least in any breadth, it would have ${ }^{15}$

${ }^{16}$ merited a category of its own. Currently, however, we are only aware of a single study $[78]^{16}$

${ }^{17}$ that tackled this challenge. Results obtained strongly justify overcommitment of processor ${ }^{17}$

${ }^{18}$ cores to vCPUs, for the case of transaction web service workloads, with the increase in ${ }^{18}$

${ }^{19}$ throughput (measured in web interactions per second, or WIPS) increasing at a faster rate ${ }^{19}$

${ }^{2 \theta}$ than power consumption. This behavior proceeds well into overcommitment ratios of pro- ${ }^{2 \theta}$

${ }^{21}$ cessor cores to vCPUs equal to 3 (three). Overcommitment of physical to VM memory is ${ }^{21}$

${ }^{22}$ not investigated. 22

$23 \quad 23$

${ }_{24}$ Investigation and classification of DPDK applications In section 4.3.2, we address the ${ }_{24}$ ${ }_{25}$ relationship between DPDK and power consumption. In the course of a public discussion ${ }_{25}$ ${ }_{26}$ in the forum offered by the North American Network Operators' Group (NANOG)[130], ${ }_{26}$ ${ }_{27}$ there emerged a need for clarity on DPDK's association with inefficiency in power con- ${ }_{27}$ ${ }_{28}$ sumption. Interest was particularly expressed in knowledge of a classification of extant ${ }_{28}$ ${ }_{29}$ DPDK applications according to their power consumption, and contribution to the code ${ }_{29}$ ${ }_{30}$ base to improve power-hungry applications.

${ }^{31}$ Modeling of power consumption by virtualized I/O Power consumption of network $\mathrm{I} / \mathrm{O}^{31}$

${ }^{32}$ has been investigated to some extent as this is central to the feasibility of network func- ${ }^{32}$

${ }^{33}$ tions decoupled from hardware. Yet while software and hardware solutions are already ${ }^{33}$ 
${ }^{1}$ available (see section 4.3.2), they require frequency and idling control (see section 3.2.2) ${ }^{1}$

${ }^{2}$ targeted to their specific operating conditions. Notably, naive DPDK runs the processor ${ }^{2}$

${ }^{3}$ core at its maximum power consumption, regardless of load. Exploitation of adaptive-rate ${ }^{3}$

${ }^{4}(\mathrm{AR})$ processing and low-power idle (LPI) should provide a means to save power while ${ }^{4}$

${ }^{5}$ processing high networking loads. However, effective control of these means is still elu- ${ }^{5}$

${ }^{6}$ sive. Despite both using Xeon Haswell microarchitectures, [95] and [64] reach opposite ${ }^{6}$

${ }^{7}$ conclusions about the feasibility of processor core C-states. The former [95] finds LPI an ${ }^{7}$

${ }^{8}$ effective means of reducing power consumption of packet forwarding (with limitation on ${ }^{8}$

${ }^{9}$ latency) while the latter [64] finds it ineffective, preferring use of the pause instruction. ${ }^{9}$

10 Furthermore: in [111], performance state transitions (P-state) are found to impose a high ${ }^{10}$

${ }^{11}$ transition latency, while in [64], P-state regulation is the preferred approach. There is scope ${ }^{11}$

${ }^{12}$ for research in the dynamic adaptation of the processor's operating state to save power. ${ }^{12}$

$\begin{array}{lr}144.3 \text { Pitfalls } & 14\end{array}$

${ }^{15} 4.3 .1$ Power consumption does not in general increase linearly with processor utilization ${ }^{15}$

${ }^{16}$ Notwithstanding advances made in identifying operating contexts that manifest sub-linear ${ }^{16}$

${ }^{17}$ power-utilization relationship [75, 82] recent publications [70, 131-138] persist in using ${ }^{17}$

${ }^{18}$ the linear model without acknowledging its limitations. The model is simple to use and has ${ }^{18}$

${ }^{19}$ some foundations in research [49]. It has three premises, described here with regard to the ${ }^{19}$

${ }^{20}$ operation of Microsoft Windows:

21 When Windows has no threads to run on a logical core, it schedules the idle thread ${ }^{21}$ 22 [139].

23 The idle thread keeps the processor in a low-power state [140]. The specific state ${ }^{23}$

24 depends on the processor's green capabilities. 24

253 In the complement (non-idle time), the processor issues instructions at a constant ${ }^{25}$

26 rate 26

${ }^{27}$ This simple model has limitations [[141], p. 6/12] [[75], p. 808]. It fails to take into ac- ${ }^{27}$

${ }^{28}$ count diverse processor operating contexts, some of which are coming to bear on current ${ }^{28}$

${ }^{29}$ use cases. Specifically, the third premise is true only to the extent to which instructions are ${ }^{29}$

${ }^{30}$ being fetched and data are being loaded from/stored to instruction and data cache, respec- ${ }^{30}$

${ }^{31}$ tively. Consider the context of $90 \%$ and greater hit ratios. At such cache hit ratios, the rate ${ }^{31}$

32 of instruction issue is expected to be narrowly distributed about its mean. By contrast: the ${ }^{32}$

${ }^{33}$ lower the hit ratio at the cache level before main memory, the lower the fraction of non- ${ }^{33}$ 
${ }^{1}$ idle time at which power consumption saturates. This saturation is strikingly illustrated in ${ }^{1}$ ${ }^{2}$ [[75], Fig. 1]. Variation of power consumption due to execution of tests from the SPEC ${ }^{2}$ ${ }^{3} \mathrm{CPU} 2000$ benchmark suite is shown. The power consumption diverges at $25 \% \mathrm{CPU}$ uti- $^{3}$ ${ }^{4}$ lization and the consumption of the processor-bound test (mesa) is greater than that of the ${ }^{4}$ ${ }^{5}$ memory-bound test (mcf) by a factor of about 2.6.

6 Another good (albeit overly broad) illustration of this pitfall is given in [[142], Fig. 5]. ${ }^{6}$

${ }^{7}$ Data on power consumption and CPU utilization under a standardized benchmark is plotted ${ }^{7}$

${ }^{8}$ for four different physical server models. None of the relationships is linear. Neither is there ${ }^{8}$

9 a single, common behavior.

10 Researchers align themselves into two groups in regard to CPU utilization. One group ${ }^{10}$

${ }^{11}$ favors (operating-)system metrics (of which CPU utilization is one metric) and the other ${ }^{11}$

${ }^{12}$ favors event counters (microarchitectural instrumentation). The arguments posed by each ${ }^{12}$

${ }^{13}$ group against the other's approach can be summarized as follows. The "system metrics"13

${ }^{14}$ group claims that the "event counters" group's work is (a) not portable (at least across mi- ${ }^{14}$

${ }^{15}$ croarchitecture families) and (b) cannot be exercised without low-level access to the host ${ }^{15}$

${ }^{16}$ (thereby, this approach cannot be exploited by user-level privileges) (see, e.g., [50], p.121 ${ }^{16}$

${ }^{17}$ and [87], p.43). The "event counters" group claims that CPU utilization is a workload- ${ }^{17}$

${ }^{18}$ dependent predictor (see, e.g., [72], p.1380) and therefore cannot be used without re- ${ }^{18}$

${ }^{19}$ training the model. Indeed, this modification to the "system-counters" approach is em- ${ }^{19}$

${ }^{20}$ ployed in [88] (section 3.2, "Runtime phase"), where, it is stated that "[b]ecause of changes ${ }^{20}$

${ }^{21}$ of VM's internal applications ... parameters must [be] recalculated automatically". Given ${ }^{21}$

${ }^{22}$ these arguments, it seems that the system metrics group argument is weak: both system ${ }^{22}$

${ }^{23}$ metrics and event counters require re-training if hardware is changed but system metrics ${ }^{23}$

${ }^{24}$ lack the granularity to discriminate between workloads (cf. [75], Fig.1). This means that ${ }^{24}$

${ }^{25} \mathrm{CPU}$ utilization can only be used as the sole predictor if it is re-trained with change in ${ }^{25}$

${ }^{26}$ workload. We deal with this problem, which we have termed the fallacy of the universal ${ }^{26}$

${ }^{27}$ model, in section 4.4.1. $\quad{ }^{27}$

${ }^{28}$ As regards use of hardware threads (Intel $®$ Hyper-Threading), we have observed that ${ }^{28}$

${ }^{29}$ various works concur on the operating context under which a linear relationship is subject ${ }^{29}$

${ }^{30}$ to the lowest error. This includes at least the following two conditions. 30

31 The processor cores are increasing their instruction issue rate in proportion to the

32 fraction of time they spend busy [75]. This implies that instruction and data cache hit ${ }^{32}$

33 ratios are high. This is simply the third premise. 
12 Only one logical core is active per physical core at any given time $[75,76,82]{ }^{1}$

2 Expressed alternatively, actual utilization must lie below half maximum utilization. ${ }^{2}$

3 The underlying cause is that activation of the second logical core employs fewer ${ }^{3}$

4 organizational units of the processor than activation of the first logical core. 4

5 The first condition is particularly problematic, as cache miss ratios are likely to be much ${ }^{5}$ ${ }^{6}$ higher in the context of virtualized environments. In such environments, the number of ${ }^{6}$ ${ }^{7}$ runnable threads is the sum of runnable threads controlled by independent operating sys- ${ }^{7}$ ${ }^{8}$ tems. Evidently, this is higher than the expected number of runnable threads on a single ${ }^{8}$ ${ }^{9}$ server instance.

10 Other evidence of this "utilization trap" is not hard to find. In [70], the compute resource ${ }^{10}$ ${ }^{11}$ is stressed using cpulimit and stress-ng. The "cpulimit" utility runs a specified process ${ }^{11}$ ${ }^{12}$ image, then pauses and resumes it until a certain percentage utilization is reached [137]. ${ }^{12}$

${ }^{13}$ The repetitive execution of a single process is highly likely to create conditions for very ${ }^{13}$ ${ }^{14}$ high instruction- and data-cache hit ratios. Such favorable hit ratios skew results towards ${ }^{14}$ ${ }^{15}$ the linear relationship between CPU utilization and power consumption. 15 16

${ }^{17}$ 4.3.2 DPDK is not intrinsically inefficient in power consumption 17

${ }^{18}$ Before proceeding to our exposition of this pitfall, we distinguish between data, control ${ }^{18}$

${ }^{19}$ and management planes. "Data plane" is a term used to refer to the infrastructural means ${ }^{19}$

${ }^{20}$ that provide the capacity for exchange of customer (or subscriber, or end-user) data. It is ${ }^{20}$

${ }^{21}$ complemented by a control plane, which refers to those means that facilitate the dynamic ${ }^{21}$

${ }^{22}$ setup, maintenance and tear-down of a functional data plane. Another complementary part ${ }^{22}$

${ }^{23}$ is the management plane. This includes the infrastructural means for a network operator to ${ }^{23}$

${ }^{24}$ configure and monitor the control plane and the data plane, as well as intervene to correct ${ }^{24}$

${ }^{25}$ faults arising in either plane. Simpler networks may have no control plane. 25

26 Research on power efficiency in DPDK applications [62-64, 66] has portrayed DPDK as ${ }^{26}$

${ }^{27}$ power inefficient. In one particular case [63] (p. 43), it is claimed that "we found that a poll ${ }^{27}$

${ }^{28}$ mode driver (PMD) thread accounted for approximately 99.7 percent CPU occupancy $\left(\mathrm{a}^{28}\right.$

${ }^{29}$ full core utilization)." The implication that seems to emerge here is that the PMD itself is ${ }^{29}$

${ }^{30}$ driving this power consumption.

31 This portrayal is problematic at best and incorrect at worst. The referenced investigations ${ }^{31}$

${ }^{32}$ of DPDK have indicated a very low power efficiency [62-64, 66], but they do not clearly ${ }^{32}$

${ }^{33}$ distinguish between responsibility of the DPDK API and the application using it (the API). ${ }^{33}$ 
${ }^{1}$ A recent, public thread [130] has emphasized the responsibility of the application devel- ${ }^{1}$

${ }^{2}$ oper in the avoidance of the naïve, "default approach" of busy polling. Such an approach ${ }^{2}$

${ }^{3}$ would, indeed, poll network IO hardware continuously [63] p. 44, truly fitting the epithet ${ }^{3}$

4"spinning-hot" [65] @26:23 ${ }^{[3]}$. However, a broader (in the sense of including industrial ${ }^{4}$

${ }^{5}$ correspondents) investigation [130] suggests that: 5

6 - contrary to claims in [63], it is the driving behaviour of OvS that is inefficient in ${ }^{6}$

7 power consumption, and 7

8 - there are simpler, technical means of throttling a polling loop, including, say the use ${ }^{8}$

9 of program code to interleave ACPI C1 states with polls according to traffic demands. ${ }^{9}$

10 These latter observations cast doubt on the claim that automated frequency control is ${ }^{10}$

${ }^{11}$ outside the scope of current frequency governors, since "the OS won't be able to distinguish ${ }^{11}$

${ }^{12}$ whether it's under a heavy load" [63], p. 45. On the other hand, savings through NUMA ${ }^{12}$

${ }^{13}$ awareness [66], (where transmit/receive port, memory and processor core are kept within ${ }^{13}$

${ }^{14}$ the same NUMA node), is affirmed in [130].

15

${ }^{16} 4.3 .3$ Research on power models without power-relevant context 16

${ }^{17}$ This pitfall traps readers who attempt to draw conclusions from published research which ${ }^{17}$

${ }^{18}$ lacks a clear specification of context relevant to power consumption. The pitfall is best ${ }^{18}$

${ }^{19}$ illustrated through examples. 19

201 Failure to emphasize context: idle power consumption vs frequency. Dependence ${ }^{20}$

21 of idle power consumption on clock frequency is context-sensitive. In [58], it is ex- ${ }^{21}$

22 plicitly stated "idle power consumption remains constant, regardless of the CPU fre- ${ }^{22}$

23 quency ... across the whole frequency range" $(1.6-2.6 \mathrm{GHz})$. The CPU is an Intel ${ }^{23}$

24 Xeon E5620. In [94] and [93], a quadratic relationship between idle power consump- ${ }^{24}$

25 tion and frequency is observed. Here, the CPU is an Intel Core i5 Haswell. In these ${ }^{25}$

26 two instances, emphasizing the restricted scope of findings would suffice to spare $\mathrm{a}^{26}$

$27 \quad$ reader from excessively broad inferences. 27

282 Failure to emphasize context: idle power consumption vs hardware and software ${ }^{28}$

29 specification. Enokido's and Takizawa's work [83] derives a power consumption ${ }^{29}$

30 model for a server while VMs run computation-bound processes. The servers used ${ }^{30}$

31 run on Intel Core i5-3230M processors. These processors are used in the mobile ${ }^{31}$

${ }^{32} \overline{[3]}_{\text {We use the @ symbol since a video is being cited and the content cited here may be found }}{ }^{32}$

${ }^{33}$ by seeking the time indicated. 
${ }^{14}$ The examples cited illustrate the importance for a researcher into power models to qualify ${ }^{14}$

${ }^{15}$ his/her results with a well-defined physical context. Research into power models involves ${ }^{15}$

${ }^{16}$ hard components and a diligent characterization thereof is essential to the acceptance of ${ }^{16}$

${ }^{17}$ work as scientific research

18

${ }^{19} 4.3 .4$ Benchmarks may skew power consumption according to their organizational 19

20 dependencies

${ }^{21}$ In section 4.3.1, it was seen that both "cpulimit" and "stress-ng" do not produce generally ${ }^{21}$

${ }^{22}$ representative measurement of power consumption. This observation is not limited to mea- ${ }^{22}$

${ }^{23}$ surement of power consumption. Use of kernels, toy programs and synthetic benchmarks ${ }^{23}$

${ }^{24}$ to measure performance has been identified as unrepresentative [[121] p. 40] of general ${ }^{24}$

25

performance. Benchmarks are standardized workload generators that are used for compar- ${ }^{25}$

${ }^{26}$ ison of computer systems for a specific class of application. Unless this application class ${ }^{26}$

${ }^{27}$ is a good representative of the application of the computer system in productive use, the ${ }^{27}$

${ }^{28}$ power consumption measured under test is not a reliable predictor of that obtained during ${ }^{28}$

${ }^{29}$ productive use. It is necessary to plan test workload generators in advance and state the lim- ${ }^{29}$

${ }^{30}$ its of validity of results. In [78], TPC-W is used, which is a transactional web benchmark ${ }^{30}$

${ }^{31}$ that can simulate the business oriented online web-servers. The MySQL++Java version of ${ }^{31}$

${ }^{32}$ TPC-W benchmark, suitable for cloud applications, is used to generate the online traffic, ${ }^{32}$

${ }^{33}$ where three different traffic profiles based on browsing, purchasing and ordering of books ${ }^{33}$ 
${ }^{1}$ are generated. The throughput measure for these servers are observed through the metric ${ }^{1}$

${ }^{2}$ Web Interactions Per Second (WIPS).

3

$4{ }^{4}$

${ }_{5}^{4.3 .5}$ Processor organization significantly impacts power consumption 5

6We illustrate this point with a wide-ranging example [147]. which compares the Intel Xeon6 7X5670 and AMD Opteron 2435.

81 Different idle loops (using no operation, pause, repetition, etc.) were tested to see ${ }^{8}$ their effect on power consumption of both systems. It was observed that the Intel ${ }^{9}$ Xeon has a loop stream detector, which disables the processor's features like fetch ${ }^{10}$ and decode. On the other hand, the AMD processor has no hint to process these loops ${ }^{11}$ efficiently; hence, it consumed more power than the Intel processor.

2 A processor consumes different amounts of power depending upon the instruction ${ }^{13}$ (such as load, addition, multiplication, etc.) and the level in the memory hierarchy ${ }^{14}$ which is accessed by the instruction.

(a) For the Xeon, data throughput of all instructions from a particular memory ${ }^{16}$ hierarchy level is almost the same but there is a difference in their power con- ${ }^{17}$ sumption. The 'load' operation consumes the lowest power compared to other ${ }^{18}$ instructions, and this holds true for all memory hierarchy levels. The reason is ${ }^{19}$ that the 'load' instruction just needs to load the content on the processor regis- ${ }^{20}$ ters whereas 'add' and 'mul' operations are more computation demanding. 21

(b) However, the AMD processor's behavior is the opposite. When the 'load' op- ${ }^{22}$ eration accesses $\mathrm{L} 1$ cache, it achieves almost one-and-a-half times the data ${ }^{23}$ throughput of other operations and hence also consumes more power. This ${ }^{24}$ difference in resource utilization is due to the different microarchitecture of ${ }^{25}$ AMD processors, where the 'load' instruction is handled by many floating- ${ }^{26}$ point pipelines. Other instructions just use a single pipeline for their opera- ${ }^{27}$ tions. Moreover, AMD processors have an exclusive cache level design, which ${ }^{28}$ requires write-back when evicting data among different cache levels. Since In- ${ }^{29}$ tel's inclusive cache design does not require this function, it consumes less ${ }^{30}$ power. Within higher memory hierarchy levels (L2 or L3 or main memory), the ${ }^{31}$ AMD's computation ('add' and 'mul') and data transfer operations ('load') de- ${ }^{32}$ liver roughly the same data throughput and consume roughly the same power. ${ }^{33}$ 
${ }^{1}$ 4.3.6 Isolation of VE for power modeling and measurement 1

${ }^{2}$ Isolation of any VE from its hardware counter parts can not be done completely [148]; thus, ${ }^{2}$

${ }^{3}$ the assumption of measuring power consumption of an individual virtual entity irrespec- ${ }^{3}$

${ }^{4}$ tive of the hardware on which it is implemented is an illusion. The virtual infrastructure ${ }^{4}$

${ }^{5}$ is composed of several components at both hardware and software level, where the effect ${ }^{5}$

${ }^{6}$ of underlying hardware, OS and VNF technology can significantly impact the power con- ${ }^{6}$

${ }^{7}$ sumption. Hence, isolation as well as modeling of power consumption for an individual ${ }^{7}$

${ }^{8}$ virtual component is difficult to obtain.

104.4 Fallacies

10

${ }^{11} 4.4 .1$ A universal power model 11

${ }^{12}$ We have suggested that the core challenge in modeling power consumption by VEs lies in ${ }^{12}$

${ }^{13}$ the number of dimensions of variability. This has been demonstrated throughout this survey, ${ }^{13}$

${ }^{14}$ where a number of generalizations have been addressed. Summarizing, the literature shows ${ }^{14}$

${ }^{15}$ that:

161 host power consumption does not generally have a linear relationship with processor ${ }^{16}$

$17 \quad 17$ utilization;

182 CPU-intensive workloads that repeatedly execute the same code skew power con- ${ }^{18}$ sumption results;

203 network-intensive workloads are power- and time-consuming because they employ ${ }^{20}$

21 emulations of network switches, but the root cause (emulation in the hypervisor soft- ${ }^{21}$

22 ware switch) disappears with SR-IOV [149], p. 5; 22

234 host saturation must be taken into account in predicting VEs' power consumption; ${ }^{23}$

245 processor utilization (an architectural attribute) is insufficient to predict host power ${ }^{24}$

25 consumption and microarchitectural attributes, such as LLC misses, are necessary to ${ }^{25}$

26 predict host power consumption even for the same level of processor utilization. 26

27 This list, while not exhaustive, amply illustrates that the several dimensions of variability ${ }^{27}$

${ }^{28}$ are significant in determination of VE power consumption. A model claiming to determine ${ }^{28}$

${ }^{29}$ power consumption as a function of fewer variables than the dimensions we have pointed ${ }^{29}$

${ }^{30}$ out must be accompanied by a scoping region that limits its use. While a precise scope ${ }^{30}$

${ }^{31}$ may be an unrealistic demand, it is essential that guidance be given about conditions of use ${ }^{31}$

32 of the model. We now illustrate this point by using two examples from the corpus. Khan ${ }^{32}$

${ }^{33}\left[[57]\right.$, p. 51] compares energy efficiency (hash/J) obtained by scheduling process threads ${ }^{33}$ 
${ }^{1}$ on additional cores, with that obtained by scheduling them on hardware threads on active ${ }^{1}$

${ }^{2}$ cores (through Intel Hyper-Threading). He shows that the former is greater than the lat ${ }^{2}$ ${ }^{3}$ ter. In apparent contrast, Enokido and Takizawa [[81], p. 279] show that for a given data ${ }^{3}$ ${ }^{4}$ transmission rate through the uplink of a software virtual switch, greater energy efficiency ${ }^{4}$ ${ }^{5}(\mathrm{~W} / \mathrm{bps})$ is obtained by operating an additional hardware thread on an active core (through ${ }^{5}$

${ }^{6}$ Intel Hyper-Threading), than operating an otherwise idle core. An important difference lies ${ }^{6}$

${ }^{7}$ in the task's processing "intensity", i.e. the rate of supply of instructions. While Khan's ${ }^{7}$ ${ }^{8}$ operations are tightly bound to the processor (cryptographic hashing), Enokido's and Tak- ${ }^{8}$ ${ }^{9}$ izawa's operations are distributed over the processor and network input/output. Without ${ }^{9}$ ${ }^{10}$ delving into detail, it is realistic to hypothesize that the average instructions per second ${ }^{10}$ ${ }^{11}$ demanded are far lower in the networking application, since transmission of a large file ${ }^{11}$ ${ }^{12}$ (as is the case here) does not take place in one processing burst. Operating time is divided ${ }^{12}$ ${ }^{13}$ between the processor and the media channel. In such a scenario, the added capacity of the ${ }^{13}$ ${ }^{14}$ same-core hardware thread suffices.

15 At the time of writing, the scope of validity (where the scope is a sub-space of the seven- ${ }^{15}$ ${ }^{16}$ dimensional space (section 4.1.1)) is typically only implicit. Notably, in [87], a "refined ${ }^{16}$ ${ }^{17}$ model" is used as a means of accurate prediction of power consumption by virtual ma- ${ }^{17}$ ${ }^{18}$ chines while running very specific benchmarks. It is also noteworthy that the authors con- ${ }^{18}$ ${ }^{19}$ template a type of onboarding process wherein "new" VM entrants to a cloud are modelled ${ }^{19}$ ${ }^{20}$ as a prerequisite to their inclusion in the power-prediction system. Indeed, such a process is ${ }^{20}$ ${ }^{21}$ already intrinsic to management and orchestration of virtual network functions. Just as the ${ }^{21}$ ${ }^{22}$ virtual deployment unit (VDU) nodes (in virtual network function descriptors (VNFDs)) ${ }^{22}$ ${ }^{23}$ store VM properties describing computer system resource demands, so can the descriptor ${ }^{23}$ ${ }^{24}$ template be extended to provide properties regarding power consumption demands. This ${ }^{24}$ ${ }^{25}$ “onboarding" is necessary since the selected predictors and modeling do not cover a suffi- ${ }^{25}$ ${ }^{26}$ ciently broad range of workload types, and a specific model must be learnt online, i.e. - on $^{26}$ ${ }^{27}$ the fly.

${ }^{28}$ On the other hand, we propose that a comprehensive power model for existing imple- ${ }^{28}$

${ }^{29}$ mentations is possible. We suggest three conditions. 29

$30 \quad 1$ Every resource that consumes power must own a counter that registers its usage, or ${ }^{30}$ lack thereof, during a specific clock cycle.

322 Usage of a specific resource during a specific clock cycle must consume a constant 32 amount of energy. This has the following corollaries. 
${ }^{10}$ In particular, if the second condition holds true, then a whole processor core cannot be an ${ }^{10}$

${ }^{11}$ atomic resource.

12

${ }^{13} 5$ Conclusion

${ }^{14}$ Energy efficiency of computer and telecommunications systems has become a major con- ${ }^{14}$

${ }^{15}$ cern over the last two decades, involving both equipment and networks. As regards the ${ }^{15}$

${ }^{16}$ computing world, the advent of datacenters and the cloud has played a major role in raising ${ }^{16}$

${ }^{17}$ awareness toward the issue of energy consumption (see, e.g., $\left.[41,150,151]\right)$. The attention ${ }^{17}$

${ }^{18}$ of Telecom operators in this respect has been directed not only to the wireless segment ${ }^{18}$

${ }^{19}$ [152], but also to the fixed network, starting with the "traditional" IP networking equip- ${ }^{19}$

${ }^{20}$ ment of special-purpose routing and switching devices $[1,153]$. The main motivations be- ${ }^{20}$

${ }^{21}$ hind both cloud and network operators have been the reduction of OPEX, but also the ${ }^{21}$

${ }^{22}$ increasing attention toward a "green" approach to the industrial evolution. 22

${ }^{23}$ Having reached a maximum by the end of the first decade of the years 2000, the attention ${ }^{23}$

${ }^{24}$ on the theme has been slightly decreasing with the advent of network virtualization and ${ }^{24}$

25 ftwarization brought forth by SDN, NFV, and the advent of $5 \mathrm{G}$ (with some notable 25

${ }^{26}$ ceptions, like [95, 154, 155], among others). As we noted in the Introduction, the reason can ${ }^{26}$

${ }^{27}$ be attributed to the belief that the increased flexibility and the consolidation of resources ${ }^{27}$

${ }^{28}$ made possible by virtualized networking components (see, e.g., [156-158]) would have ${ }^{28}$

${ }^{29}$ sufficed not only to compensate the increased energy consumption caused by the adoption ${ }^{29}$

${ }^{30}$ of general-purpose hardware, but also to curb it in general. We noticed that this is not the

${ }^{31}$ case. However, as noted in [159], the interest in energy efficiency is rising again with the ${ }^{31}$

${ }^{32}$ evolution toward the 6 th generation mobile network $(6 \mathrm{G})$, where the very ambitious goal ${ }^{32}$

${ }^{33}$ of $1 \mathrm{Tbit} / \mathrm{J}$ is being set. Moreover, network operators are looking with increasing interest to ${ }^{33}$ 
${ }^{1}$ the business opportunities offered not only by network softwarization and by the stricter in- ${ }^{1}$

${ }^{2}$ tegration between the mobile and fixed segment, but also by the deployment of computing ${ }^{2}$

${ }^{3}$ capabilities in the network edge brought forth by Mobile Edge Computing (MEC). 3

4 These considerations have led us to address specifically modelling and measurement of ${ }^{4}$

${ }^{5}$ power consumption in virtualized computing and networking environments and to concen- ${ }^{5}$

${ }^{6}$ trate the scope of our investigation to what is more pertinent to the Telco cloud. In doing ${ }^{6}$

${ }^{7}$ this, we have decided to adopt the IPAD method of thematic analysis, in order to attempt to ${ }^{7}$

${ }^{8}$ produce a quantitative categorization of the multi-faceted research aspects that are scattered ${ }^{8}$

${ }^{9}$ in the literature. One of our main reasons for the adoption of this methodology is grounded ${ }^{9}$

${ }^{10}$ in the additional complexity that lies in the analysis of the energy consumption caused by ${ }^{10}$

${ }^{11}$ VEs that act in telecommunication systems, with respect to those acting in the computing ${ }^{11}$

${ }^{12}$ world. On one hand, it is true that, as softwarization advances in networking and telecom- ${ }^{12}$

${ }^{13}$ munication systems, their characteristics become closer to computer systems' ones; on the ${ }^{13}$

${ }^{14}$ other hand, the nature of teletraffic and the distributed essence of networking add complex- ${ }^{14}$

${ }^{15}$ ity to the functionality and interaction of processing units that have to perform the spatial ${ }^{15}$

${ }^{16}$ transport of information or to deal with both transport and in-network computation. 16

17 Among the themes that our survey has brought us to identify, besides the review of the ${ }^{17}$

${ }^{18}$ state of the art that has led to our taxonomy and the related statistics, we have tried to high- ${ }^{18}$

${ }^{19}$ light the main research domains, along with pitfalls, possible gaps and fallacies. As regards ${ }^{19}$

${ }^{20}$ the first, we have pointed out three major interests addressed in the literature within the ${ }^{20}$

${ }^{21}$ scope of our investigation: i) predictive models of real-time power consumption, ii) inte- ${ }^{21}$

${ }^{22}$ gration of computing and networking systems with emphasis on energy efficiency and iii) ${ }^{22}$

${ }^{23}$ high-performance, power-efficient packet I/O frameworks. All problem categories we iden- ${ }^{23}$

${ }^{24}$ tified within these broader domains touch one or more of a set of seven main variables that ${ }^{24}$

${ }^{25}$ may affect power consumption by virtual entities and the ensuing model representations: ${ }^{25}$

${ }^{26}$ workload type, characteristics of the virtualization agent (VM or container), host machine ${ }^{26}$

${ }^{27}$ resources and architecture, temperature, operating frequency, attribution of a fraction of ${ }^{27}$

28 consumed power to individual VEs, and mutual influence of concurrent VEs. 28 consumed power to individual VEs, and mutual influence of concurrent VEs.

29 Among the major pitfalls that could be noted from our thematic analysis, we highlight ${ }^{29}$

${ }^{30}$ here the often unacknowledged limitations of the widely used linear models, the failure ${ }^{30}$

${ }^{31}$ to precisely identify the physical context in some experimental research, the influence of ${ }^{31}$

${ }^{32}$ synthetic workload generators on measurements, and the sometimes overlooked relevance ${ }^{32}$

${ }^{33}$ of processor organization on power consumption measurements. We have also pointed out ${ }^{33}$ 
${ }^{1}$ the unavoidable need to precisely identify the scope and limitations of models and the ${ }^{1}$

${ }^{2}$ fallacy of the quest for a "universal" power model.

4 Evolution of the research space on power consumption in virtualized the environments 4 ${ }_{5}$ composing the Telco cloud now suggests directions for further development of power mod-5 6els: 21 standing and mitigating power consumption of virtual entities is bound to grow in the near ${ }^{21}$ ${ }^{22}$ future (and - we may add - especially in the Telco cloud), owing to the increased soft- ${ }^{22}$ ${ }^{23}$ warization process in networking and to the relevance of edge computing in the roadmap ${ }^{23}$ ${ }^{24}$ beyond 5G. Attention should be increasingly shifting toward indirect measurement of the ${ }^{24}$

25 . power consumption by VEs and the development of prediction models based on the use ${ }^{25}$ ${ }^{26}$ of Artificial Intelligence / Machine Learning (AI/ML) techniques. As we mentioned in our ${ }^{26}$ ${ }^{27}$ sub-section on research gaps to be addressed, modelling in particular the power consump- ${ }^{27}$ ${ }^{28}$ tion of containers (and Metal-as-a-Service approaches, as well), along with that of virtual- ${ }^{28}$ ${ }^{29}$ ized I/O should play a relevant role. In this vision, we tend to agree with the architectural ${ }^{29}$ ${ }^{30}$ paradigm referred to as Smart Green Network Concept in the latest version of the Strategic ${ }^{30}$ ${ }^{31}$ Research and Innovation Agenda (SRIA) 2021-27, recently issued by NetWorld2020 [160], ${ }^{32}$ where AI-as-a-Service (AIaaS) occupies a relevant position above a unified controllability ${ }^{32}$ ${ }^{33}$ layer and below a cooperative services layer offered to verticals. 24 5

3

Division of the problem into (a) a modeling concern: (i) what components to include, (ii) what workload(s) to consider, (iii) what state factors (temperature, frequency, performance $\&$ idle states) to choose to be accounted for. 年

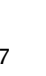
8

9

30
31
32
33


${ }^{1} 6$ Appendix - Applications ${ }^{1}$

${ }^{2}$ Interest in measuring and modeling power consumption by virtual machines and contain- ${ }^{2}$

${ }^{3}$ ers derives from the relationship which power consumption has with the cost of operations ${ }^{3}$

4 of information and communication technology (ICT) infrastructure. This statement is rel- ${ }^{4}$

${ }^{5}$ evant to infrastructure provision across the scale, whether in Cloud datacenters or virtu- ${ }^{5}$

${ }^{6}$ alized infrastructure running in points of delivery within the access segment of a public ${ }^{6}$

${ }^{7}$ telecommunications network (PTN). Operation, administration, maintenance and manage- ${ }^{7}$

${ }^{8}$ ment (O\&M) [17] of ICT infrastructure using guidance from knowledge of power con- ${ }^{8}$

${ }^{9}$ sumption is referred to as power-aware O\&M. Power-aware O\&M of redundant infrastruc- ${ }^{9}$

${ }^{10}$ ture hinges on models of physical infrastructure's power consumption as a function of $\mathrm{VM}^{10}$

${ }^{11}$ workload (measured in terms of architectural or microarchitectural entities). Therefore, ${ }^{11}$

${ }^{12}$ application of modeling and measurement lies in the power-aware O\&M of virtualized ${ }^{12}$

13 environments.

14 Power-aware O\&M has the objective of optimization of such O\&M. We distinguish be- ${ }^{14}$

${ }^{15}$ tween optimization and minimization through service constraints: optimization is the mini- ${ }^{15}$

${ }^{16}$ mization of costs subject to constraints on operations explicitly or implicitly obtained from ${ }^{16}$

${ }^{17}$ service-level agreements (SLAs). A well-known example of the impact of SLAs on power ${ }^{17}$

${ }^{18}$ consumption regards the use of redundant equipment to meet service availability require- ${ }^{18}$

${ }^{19}$ ments. Service availability (an important parameter of SLAs) depends critically on the ${ }^{19}$

${ }^{20}$ absence of single points of failure (SPOFs). The redundant physical equipment deployed ${ }^{20}$

21 to avoid SPOFs increases power consumption. 21

${ }^{22}$ Here, we briefly survey research into power awareness of O\&M in virtualized environ- ${ }^{22}$

${ }^{23}$ ments, where such O\&M have significant power consumption that must be measured and ${ }^{23}$

${ }^{24}$ modelled. Five concerns emerge:

251 Creation, deletion, startup, shutdown, hibernation, resumption and migration oper- ${ }^{25}$

26 ations characterize the dynamicity of virtualized environments. The significance of 26 27 their overhead in power consumption must be quantified to determine its impact on ${ }^{27}$

28 energy efficiency. In particular, migration's cost is not trivial, although acceptable. ${ }^{28}$ [84].

2 Furthermore, in order to operate a commercial virtualized environment, heterogene- ${ }^{30}$ ity in both VMs and hosts must be characterized in models. Primarily, heterogeneity ${ }^{31}$ refers to variability in allocation of resource quantities: a virtualized environment ${ }^{32}$ supports diverse VM sizes. Secondarily, there are also implementation differences in ${ }^{33}$ 
1 physical machines and these differences manifest themselves in the power models. ${ }^{1}$

2 This dimension of variability must be included in modeling to support frameworks ${ }^{2}$ of O\&M.

43 Pay-per-use is one of the key tenets of Cloud Computing. Implementation of this ${ }^{4}$

5 tenet depends upon the facility to account for resource usage. This facility may be

6 passed on to the consumer or reserved by the provider for internal cost control. $\mathrm{We}^{6}$

7 observe an evolution of research into comprehensive frameworks capable of automat ${ }^{7}$

$8 \quad$ ing the transformation of resource usage into cost. 8

94 Real-time control of power consumption is concerned with scheduling VEs (a) to ${ }^{9}$ minimize consumption or (b) to cap it.

115 The use of models to reason about macroscopic changes to digital services and/or ${ }^{11}$ systems is concerned with the exploration of the impact of alternatives in service de- ${ }^{12}$ livery or system implementation. We refer to such applications of models as macro- ${ }^{13}$ scopic analyses.

15 The first two concerns are part of the broader scope of a Network Function Virtualization ${ }^{15}$

${ }^{16}$ Management and Orchestration framework (NFV-MANO) [161] whereas the third concern ${ }^{16}$

${ }^{17}$ is part of a Business Support System (BSS). The fourth concern is part of the scope of a ${ }^{17}$

${ }^{18}$ Virtual Infrastructure Manager (VIM). The fifth concern is that of strategic management. ${ }^{18}$

${ }^{19}$ We divide our review of application research into these three system classes (NFV-MANO ${ }^{19}$

${ }^{20}-6.1$, VIM - 6.2, BSS - 6.3) and macroscopic analyses (6.4).

${ }^{22} 6.1$ Management and Orchestration

${ }^{23}$ We consider a sample of works that deal with power-aware VM migrations $\left[84,134,162,{ }^{23}\right.$

$\left.{ }^{24} 163\right]$. Their approach is divided into two parts. The first part regards basic research, where ${ }^{24}$

${ }^{25}$ the researchers develop a power model to guide the second part. This latter part may be ${ }^{25}$

${ }^{26}$ described as an approach to applied research, where the power model(s) is(are) used to ${ }^{26}$

${ }^{27}$ quantify the impact of migrations on power consumption. In order to present the sample, ${ }^{27}$

${ }^{28}$ we apply and present a first iteration (see [45, sub-section 4.2] for some clarification of ${ }^{28}$

${ }^{29}$ what we mean by "iteration" here) of the IPAD protocol on RUs that employ models to ${ }^{29}$

${ }^{30}$ improve the power or energy efficiency of VM migrations. 30

31 In [84]:

32 Problem: How does VM migration affect power consumption? 32

332 Approach 33 
(a) Basic research

i. Workload Model: Historical data about static and periodic cloud workload ${ }^{2}$ patterns was collected and used in an ARIMA model to predict workload. ${ }^{3}$ The predicted workload was transformed into a prediction of power con- ${ }^{4}$ sumption through the linear/polynomial model of power consumption as $\mathrm{a}^{5}$ function of virtual processor utilization.

ii. Power Model: A relationship was obtained, through linear or polynomial ${ }^{7}$ regression, between the workload (utilization of virtual processors hosted ${ }^{8}$ by a physical machine), and its power consumption.

(b) Applied research: Applied research: The approach taken is to reduce frequency ${ }^{10}$ of migration. The SLA is respected by bounding server power below a thresh- ${ }^{11}$ old. Server power consumption is used since the relationship between workload ${ }^{12}$ and host power consumption is determined during basic research, and mea- ${ }^{13}$ surement of host power consumption is less invasive and less demanding than ${ }^{14}$ measurement of several individual VM workloads.

(a) The migration algorithm does not migrate the $\mathrm{VM}$ as soon as the high $\mathrm{CPU}^{17}$ utilization peak is detected. The algorithm waits for some time and if the high ${ }^{18}$ utilization persists, then the VM is migrated. This facilitates improved energy ${ }^{19}$ efficiency through optimal migration of heterogeneous virtual machines under ${ }^{20}$ different workloads.

(b) VMs can be migrated without violating performance terms of the Service-Level ${ }^{22}$ Agreement (SLA) by limiting the server's power consumption below a given ${ }^{23}$ threshold. This framework considers the heterogeneity of virtual machines and ${ }^{24}$ predicts the variation in key parameters such as power, performance and migra- ${ }^{25}$ tion cost, for different usage and size of the VM.

274 Limitations: The workload prediction model is limited to estimating the precise ${ }^{27}$ power cost of periodic workloads; therefore, it might not be useful for a dynamic ${ }^{28}$ scenario.

${ }^{31} 6.1 .1$ Orchestration in Datacenters

${ }^{32}$ Deploying a single service in a single host is fairly simple to manage. However, these ${ }^{32}$ ${ }^{33}$ days, in large datacenters and cloud networks, no single server is serving a single appli- ${ }^{33}$ 
${ }^{1}$ cation. Virtualization has opened new ways of service provisioning and application pro- ${ }^{1}$

${ }^{2}$ cessing on a single server where several users can run a number of different applications, ${ }^{2}$

${ }^{3}$ each isolated from the other. Optimal resource management and task scheduling in $\mathrm{such}^{3}$

${ }^{4}$ scenarios is impractical to achieve using traditional scheduling schemes such as round ${ }^{4}$

${ }^{5}$ robin, first come first served, etc. Heuristic or meta-heuristic based optimization mod- $^{5}$

${ }^{6}$ els are usually preferred in heterogeneous networks for this purpose. However, in some ${ }^{6}$

${ }^{7}$ cases, even these methods can take a significantly long time to find the optimal solution ${ }^{7}$

${ }^{8}$ and consequently consume more energy. Considering these issues, many researchers pro- ${ }^{8}$

${ }^{9}$ posed energy-efficient management schemes [164-166] and orchestration tools [167-169] ${ }^{9}$

${ }^{10}$ to manage resources effectively. These commercial tools help to manage large networks of ${ }^{10}$

${ }^{11}$ clustered applications, multiple datacenters, public and private clouds, etc., and seem to be ${ }^{11}$

${ }^{12}$ a robust, energy efficient choice for VM consolidation and load balancing. 12

13 We again apply and present a first iteration of the IPAD protocol; in scope here are RUs ${ }^{13}$

${ }^{14}$ that employ models to obtain strategies and management schemes. 14

15 In [165]: 15

161 Problem: Develop an energy efficient management scheme for a large network with ${ }^{16}$

$17 \quad 17$

182 Approach: Cloud application behavior at a given instant is considered. Performance ${ }^{18}$

19 state and power state are adapted to meet the application's demand. Specifically: ${ }^{19}$

20 DVFS and VM consolidation are used in the proposed orchestrator to reduce power ${ }^{20}$

21 consumption. 21

223 Developments 22

23 (a) developed an energy-efficient cloud orchestrator (e-eco) which opts for an ap- ${ }^{23}$

$24 \quad$ propriate management scheme; 24

(b) the proposed strategy allows VM consolidation for hosts running at low $\mathrm{CPU}^{25}$ usage rate when images of VMs are centralized. It is an energy efficient tech- ${ }^{26}$ nique as it only requires memory pages to transfer through the network. How- ${ }^{27}$ ever, when VM images are only available where they were initialized, it is ${ }^{28}$ preferred to reduce the processor's frequency using DVFS to save energy. ${ }^{29}$

(c) In comparison to previous studies, e-eco reaches the same energy saving rates ${ }^{30}$ with far less impact on the application performance. Performance trade-off be- ${ }^{31}$ tween power saving and SLA violation was about $25 \%$ and $6 \%$, respectively. ${ }^{32}$ 
11 Problem: Energy efficient orchestration tool for VM consolidation in cloud. 1

22 Approach

3 (a) Heuristics and meta-heuristics are applied to obtain algorithms based on ${ }^{3}$

${ }^{24} 6.1 .2$ Real-time control: Schedulers

${ }^{25}$ In automated virtualized environments, scheduling is a set of activities in the control plane, ${ }^{25}$

${ }^{26}$ guided by a management policy, that instantiates, monitors, migrates and terminates the ${ }^{26}$

${ }^{27}$ virtual machines and/or containers which encapsulate the workload. One useful implemen- ${ }^{27}$

${ }^{28}$ tation of a power-aware scheduler would operate either to minimize a (cost) function of ${ }^{28}$

${ }^{29}$ power under service constraints or a multi-objective function of power and some key per- ${ }^{29}$

${ }^{30}$ formance indicator.

31 In [170], 31

$32 \quad 1$ Problem: how to schedule dynamically ariving tasks among VM. 5

(b) Input regarding state of resources (idle/used) is required to tackle consolidation. ${ }^{6}$

(a) An optimal solution for VM placement and its network paths (required band- ${ }^{8}$

(b) Different approaches for consolidation categorized as max power saving (ag- ${ }^{10}$

(c) Consolidation strategies are assessed. For example, in critical and latency sen- ${ }^{13}$ sitive applications, a less-aggressive consolidation approach (i.e. using maxi- ${ }^{14}$ mum available servers) shows better performance, although energy efficiency ${ }^{15}$ is compromised to some extent in this case. Aggressive consolidation (i.e. al- ${ }^{16}$ locate maximum VMs to a single server) saves the maximum energy while ${ }^{17}$ maintaining the performance when VMs are not fully (100\%) utilized. $\quad 18$

(d) VM consolidation (supported by context information from users) helps in re- ${ }^{19}$ ducing the power consumption if some idle VMs are present in the system. ${ }^{20}$ The algorithm groups all idle VMs together and places them on the server with ${ }^{21}$ $\begin{array}{ll}\text { lowest power state to reduce power consumption. } & 22 \\ 23\end{array}$

2 25 
${ }^{31}$ 6.1.3 Real-time control: Server Power Capping

${ }^{32}$ We demonstrate how models are applied to server power capping by parsing one relevant ${ }^{32}$

${ }^{33} \mathrm{RU}$ using the IPAD review protocol.

(b) Heuristics and meta-heuristics are applied to obtain algorithms based on vari- $^{2}$

(a) An algorithm is proposed, that combines classical Round Robin and Weighted ${ }^{5}$ Round Robin for scheduling of dynamically arriving tasks among VMs. $\quad 6$

(b) Furthermore, Improved Weighted Round Robin (IWRR) is proposed. The static ${ }^{7}$ scheduler in IWRR considers the task length, priority, resource capacity (sup- ${ }^{8}$ ply) and load (demand) on VMs to allocate a task to an appropriate VM. 9

(c) To further optimize system performance, a dynamic scheduler monitors the ${ }^{10}$ system status. It balances the load among VMs on completion of each task. $\operatorname{In}^{11}$ this way, unbalanced resource usage that may occur because of poor estimation ${ }^{12}$ of task completion time or processing delay can be fixed immediately. 13 In [91],

1 Problem: how to deal with dynamic load and resource management in virtualized ${ }^{15}$ environments.

(a) Model bias favors a discrete state transition model, employing the following ${ }^{18}$ state variables:

ii. number of VMs

iii. frequency of operation of $\mathrm{vCPU}$

(b) Employs a non-linear power model.

20 21 22

3 Developments: A predictive controller for virtual systems based on a non-linear ${ }^{24}$ power model and a discrete state transition model is obtained. The processor is ${ }^{25}$ switched among the various states during runtime, to balance the workload among ${ }^{26}$ different virtual machines.

4 Limitations: This model is unsuitable for a large state space, as this would make ${ }^{28}$ scheduling and design complex. 
(a) Power model: employs a model that relates the effect of hyper-threading and ${ }^{4}$ processor fan speed to VM power consumption.

(b) Attribution: to attribute fan power consumption fairly among all VMs, each VM's share is found using the dynamic power behavior for each virtual machine.

(c) Migration: a host near its power threshold is detected with assigned maximum and minimum power of the server. The VM to migrate is chosen by monitoring ${ }^{10}$ its dynamic power and its effect on server power after migration. The virtual ${ }^{11}$ machine selected for migration is that with the least dynamic power necessary ${ }^{12}$ to reduce its host's power consumption below an upper threshold. The VM ${ }^{13}$ consuming least dynamic power is chosen as this correlates positively with the least time to migrate.

3 Developments 11

14

(a) Modeling: the study found that the varying power consumption of a fan changes ${ }^{17}$ the power consumption of a server. 18

(b) Results show that this power measurement scheme for virtual machines is ac- 19 curate enough to support effective server power capping through the use of $\mathrm{VM}^{20}$ migration. 
${ }^{1}$ processor or consumed resources. These models charge equally for users having similar ${ }^{1}$

${ }^{2} \mathrm{VM}$ configuration even if they utilize different CPU percentages. ${ }^{2}$

3 For example, consider the case where two users use a similar VM in the cloud but run ${ }^{3}$

${ }^{4}$ different applications, say, a web server and a CPU-intensive workload, respectively. The ${ }^{4}$

${ }^{5} \mathrm{VM}$ running the web server will use around 5\% of the CPU whereas the other VM might ${ }^{5}$

${ }^{6}$ use $90 \%$ of the CPU resources. 'Pay-as-you-go' will charge the same price to both users ${ }^{6}$

$\begin{array}{ll}{ }^{7} \text { despite both having distinct workload profiles and consequently, energy consumption. } & 7\end{array}$

8 This is gratingly unjust and calls for remedial action. Indeed, significant effort has been ${ }^{8}$

${ }^{9}$ brought to bear in at least two channels of effort: the academic, wherein modeling and ${ }^{9}$

${ }^{10}$ measurement is the primary means to enable a fair billing application, and the industrial, ${ }^{10}$

${ }^{11}$ where, concurrently, efforts to harness academia's (and industry's) developments in this ${ }^{11}$

${ }^{12}$ space are under way in the form of a green abstraction layer for virtualized environments. ${ }^{12}$

${ }^{13} \mathrm{~A}$ brief review of these two channels follows.

14

${ }_{15}$ Development within academia We observe firstly several works directed specifically at ${ }_{15}$ ${ }_{16}$ coupling power consumption, virtual resource usage and accounting for costs. Here, the ${ }_{16}$ ${ }_{17}$ intention is not solely to relate virtual resource usage and the ensuing power consumption $_{17}$ ${ }_{18}$ but rather a more ambitious infrastructural combination of measurement and cost into $\mathrm{a}_{18}$ $\begin{array}{ll}{ }_{19} \text { single system. } & 19\end{array}$

20 In [171], 20

211 Problem: how to move away from fixed billing, based on VM size and duration of ${ }_{21}$ usage, to billing cost for service based on CPU/resource usage. 22

2 Approach: a cost model named 'Proportional-Shared Virtual Energy (PSVE)' is con- ${ }_{23}$ ceived of for VM instances. It is a composite of CPU energy consumption and tradi- ${ }_{24}$ tional commodity prices, such as management and operational cost of hypervisor. $\quad 25$

(a) Considering the CPU usage knowledge available at hypervisor, a proportional ${ }_{27}$ energy-cost sharing model for a VM can be developed.

(b) The proposed model can also be used to evaluate the efficiency of services ${ }_{29}$ by service providers and to develop optimized applications to reduce energy $y_{30}$ consumption.

${ }^{32}$ Industrial Development/Green Computing Industrial effort is visible in extension of the ${ }^{32}$

${ }^{33}$ European standard for green abstraction of non-virtualized environments [172] to virtual- ${ }^{33}$ 
${ }^{1}$ ized environments. This second version of the standard [173] for a green abstraction layer ${ }^{1}$

${ }^{2}$ aims to facilitate the development of software for automation of control and accounting ${ }^{2}$ ${ }^{3}$ functions. It is tightly integrated into ETSI's group of specifications on management and ${ }^{3}$ ${ }^{4}$ orchestration of virtual functions, e.g. [174], adhering to the architecture described therein. ${ }^{4}$ ${ }^{5}$ Along with improving architecture for green computing, use of renewable energy sources ${ }^{5}$ ${ }^{6}$ (RES) to operate cloud datacenters is also proposed. Renewable energy generators using ${ }^{6}$ ${ }^{7}$ solar panels were installed at datacenters located in different geographical locations [175]. ${ }^{7}$ ${ }^{8}$ These datacenters have been using a flexible load management tool, EcoMultiCloud [176], ${ }^{8}$ ${ }^{9}$ that balances the load considering objectives such as energy cost variation and renewable ${ }^{9}$ ${ }^{10}$ energy production in the region at a particular time. Performance evaluations show that ${ }^{10}$ ${ }^{11}$ use of RES in a distributed datacenter network can save energy cost significantly without ${ }^{11}$ ${ }^{12}$ compromising on performance.

${ }^{15}$ Digital services (online news sites, video over-the-top, voice-over-IP as in $4 \mathrm{G}$ cellular ${ }^{15}$ ${ }^{16}$ networks) and telecommunications systems (e.g. centralized/cloud radio access network, ${ }^{16}$ ${ }^{17}$ evolved packet core service gateway) are the object of several optimization studies that are ${ }^{17}$ ${ }^{18}$ too broad in scope to afford the computational weight of granular power models of the ${ }^{18}$ ${ }^{19}$ kinds described in the paragraphs of section 3.3.1. These studies estimate power consumed ${ }^{19}$ ${ }^{20}$ to meet demand for a service-/system-specific workload. The objective is the investigation ${ }^{20}$

${ }^{21}$ of the impact of infrastructural alternatives on power consumed to meet the temporally $-^{21}$ ${ }^{22}$ and possibly geographically - variable load profile. An understanding at a macroscopic ${ }^{22}$ ${ }^{23}$ level is sought of the effect of some change in the composition of the digital service, on the ${ }^{23}$

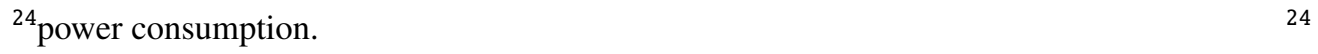

25 The approach towards understanding includes: 25

26 - identification of a unit of workload, and 26

27 - a temporal (and possibly geographical) profile of the demand (load) for the service ${ }^{27}$

${ }^{28}$ We have referred to a complex characterization of workload as a means to "quickly pro- ${ }^{28}$

29 ceed to a good estimate of the power or energy required to produce the workload" ( 29

${ }^{30}$ tion 4.1.5). Such an approach abstracts real-time operation of a computing or telecommuni- ${ }^{30}$

${ }^{31}$ cations (sub-) system, by reducing the characterization of the power-performance relation- ${ }^{31}$

${ }^{32}$ ship of its major components to a conservative ratio of power consumed to produce a unit ${ }^{32}$

${ }^{33}$ of workload. Therefore, the aim of such research is not to facilitate real-time energy-aware ${ }^{33}$ 
${ }^{1}$ control (through the development of models of real-time, dynamic power consumption) ${ }^{1}$

${ }^{2}$ but to justify the use of an (alternative) architecture, organization or implementation. Two ${ }^{2}$

${ }^{3}$ examples are described briefly below.

3

4 In [31], the concern regards whether a telecommunications system built around virtual ${ }^{4}$

${ }^{5}$ network functions (VNFs) can readily be claimed more energy efficient than one built ${ }^{5}$

${ }^{6}$ around physical network functions (PNFs). A case study is undertaken with a focus on ${ }^{6}$

${ }^{7}$ the evolved packet core's (EPC) serving gateway (SGW). Part of the modus operandi of ${ }^{7}$

${ }^{8}$ this application of power modeling is explicitly declared in [31]: 8

91 a mathematical model, and 9

102 the energy consumed to meet 10

113 the performance levels demanded of the SGW. 11

12 The "performance levels demanded" are derived from the workload. Therefore, a profile ${ }^{12}$

${ }^{13}$ of workload is required, to determine the performance of the system components over $\mathrm{a}^{13}$

${ }^{14}$ temporal (and possibly geographical) range. The conjunction of a temporally unfolding ${ }^{14}$

${ }^{15}$ workload and power consumed to meet this workload lead to the energy consumption. ${ }^{15}$

${ }^{16}$ Hence, a figure of merit can be ascribed: the energy consumed to process the workload is ${ }^{16}$

${ }^{17}$ an indicator of energy efficiency. 17

18 The missing piece in this puzzle is how to "fit" the workload into the investigated (sub-) ${ }^{18}$

${ }^{19}$ system (this is the first phase) and therefrom, to its power consumption (this is the second ${ }^{19}$

${ }^{20}$ phase). Herein lies the key abstraction inherent to the macroscopic analysis.

21 Phase 1: the maximum throughput of a server bridges the gap between hardware and ${ }^{21}$

22 workload 22

232 Phase 2: transforms a performance level into a power consumption. 23

${ }^{24}$ We identify this two-phase transformation, from workload to power consumption, as $a^{24}$

${ }^{25}$ model in the highest of the three levels of abstraction into which we have classified power ${ }^{25}$

${ }^{26}$ models earlier (section 4.1.5). 26

27 In this particular example, the maximum throughput is bound (reasonably, albeit sweep- ${ }^{27}$

${ }^{28}$ ingly) to the maximum power consumption of the implementing device. The VNF is im- ${ }^{28}$

${ }^{29}$ plemented on a commodity-off-the-shelf (COTS) server. The PNF is implemented as a ${ }^{29}$

30 chassis-bound SGW

31 In [123],

32 - the telecommunications sub-system is a centralized- (cloud-) radio access network ${ }^{32}$

$33(\mathrm{C}-\mathrm{RAN})$; 33 (C-RAN); 
2

${ }^{4}$ modulation, mapping and multiple-input-multiple-output processing, followed by a Fast ${ }^{4}$

${ }^{5}$ Fourier Transform); there are also the inverse four reception steps.

${ }^{6} 1$ First phase: each step loads a single vBBU with a certain amount of a unit of opera- ${ }^{6}$ mated. Here, the ultimate objective is not the power consumption estimate itself, but a cessing to the vBBUs in a pool.

18

\section{${ }^{19} 7$ Appendix - The Engineer's Kit Bag}

${ }^{20} 7.1$ Observations on tools

${ }^{21}$ This section presents the researcher with a summary on the usability of different com- $^{21}$

${ }^{22}$ ponents required for designing a power model. We cover choice of workload and relevant ${ }^{22}$

${ }^{23}$ benchmarks, as well as various tools and models frequently used in research studies. Fig. $24^{23}$

24 - Fig. 26 show a frequency analysis of the occurrence of these components, extracted from ${ }^{24}$

${ }^{25}$ the studies included in this survey. Furthermore, reference to the corresponding research ${ }^{25}$ 26 studies can be found in Table 7 - Table 8 .

27

${ }^{28}$ 7.1.1 Workload Generators/Benchmarks

${ }^{29}$ Reliability of studies, analyses and models depends heavily on (a) the environment and ${ }^{29}$

${ }^{30}$ (b) workload considered. Setting up the environment is simple compared with finding an ${ }^{30}$

${ }^{31}$ appropriate workload. A benchmark suite that stresses different aspects of processor (such ${ }^{31}$

${ }^{32}$ as computing, memory, and network) in a ratio similar to, and has a computing requirement ${ }^{32}$

33 similar to that of real-time applications in datacenters should be considered 33

similar to that of real-time applications in datacenters should be considered. 
1 The Standard Performance Evaluation Corporation (SPEC) provides a number of bench- ${ }^{1}$

${ }^{2}$ marks for different applications and performance evaluation. SPEC CPU benchmarks are ${ }^{2}$ ${ }^{3}$ industry standards and well accepted in research studies [92]; this is affirmed by our ${ }^{3}$ ${ }^{4}$ frequency analysis (fig. 24). For cloud applications, benchmark suite SPEC Cloud IaaS ${ }^{4}$ ${ }^{5}[92,177]$ stresses resource provisioning, storage, network resources and computation. To ${ }^{5}$ ${ }^{6}$ generate a server workload, SPEC Web [178] can be used. An energy efficiency benchmark ${ }^{6}$

${ }^{7}$ for scalable virtual networks, based on SPEC power methodology [177], is also designed ${ }^{7}$ ${ }^{8}$ specifically for VNF applications.

9 Cloud providers and cloud sites are also being evaluated through benchmarks. A re- ${ }^{9}$ ${ }^{10}$ port [179] shows that the BitCurrent benchmark was used to evaluate nine different cloud ${ }^{10}$ ${ }^{11}$ providers. Another study used CloudHarmonics [180] to evaluate 144 different cloud sites. ${ }^{11}$ ${ }^{12}$ In an evaluation of cloud performance models and different cloud benchmark suites on ${ }^{12}$ ${ }^{13}$ Amazon EC2 [181], BenchCloud was found to be one of the five widely-used benchmarks. ${ }^{13}$ ${ }^{14}$ BenchCloud was developed in academia at the University of Southern California (USC). ${ }^{14}$ ${ }^{15}$ It contains a workload from real-time social media applications for big data processing. ${ }^{15}$

${ }^{16}$ Another benchmark from academia is CloudSuite, developed at EPFL, Lausanne, Switzer- ${ }^{16}$

${ }^{17}$ land. The workload pattern for CloudSuite is based on media streaming, web services ${ }^{17}$

${ }^{18}$ and data/graphic analytics. Two industry-based workload patterns generated by Yahoo and ${ }^{18}$

${ }^{19}$ Transaction Processing Performance Council (TPC) are YCSB and TPC-W, respectively. ${ }^{19}$

${ }^{20} \mathrm{TPC}-\mathrm{W}$ is a webserver-based benchmark; YCSB is a synthetic workload for cloud ser- ${ }^{20}$

${ }^{21}$ vice evaluation developed by Yahoo. HiBench is a workload developed specifically to run ${ }^{21}$

${ }^{22}$ Hadoop programs in clouds.

${ }^{23}$ Selection of a benchmark suite for evaluating modern processors must consider support ${ }^{23}$

${ }^{24}$ for processor features, e.g. multi-core/single core, simultaneous multi-threading (e.g. Intel ${ }^{24}$

${ }^{25}$ Hyper-Threading) and dynamic overclocking (e.g. Intel Turbo Boost), etc. Workload pat- ${ }^{25}$

${ }^{26}$ terns of different benchmarks may also include the facility to select between serial stressing ${ }^{26}$

${ }^{27}$ and parallel stressing. We observe that the analysis of multi-core systems using several in- ${ }^{27}$

${ }^{28}$ stances of serial benchmark suites (e.g. SPEC CPU) is not preferred: such instances will be ${ }^{28}$

${ }^{29}$ treated as multiple applications [182]. This further increases the energy consumption due ${ }^{29}$

${ }^{30}$ to unexpected contention for shared resources. Therefore, parallel benchmark suites such ${ }^{30}$

${ }^{31}$ as NPB-MPI, PARSEC, etc., should be used to analyze the performance and energy/power ${ }^{31}$

${ }^{32}$ consumption of parallel processing. PARSEC is a benchmark which incorporates work- ${ }^{32}$

${ }^{33}$ loads suited to several different computer classes (e.g. servers, workstations) $[93,94,182]{ }^{33}$ 


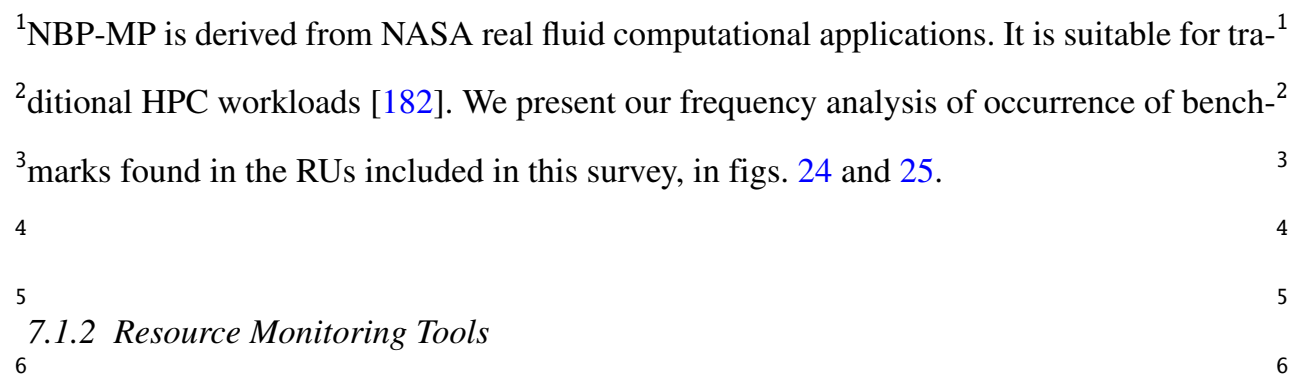
${ }_{7}$ Several methods of monitoring performance are available which either observe parameters ${ }_{7}$ ${ }_{8}$ inside the VE or through its host [187]. Methods that monitor VE performance from outside ${ }_{8}$ $9_{9}^{\mathrm{a} V E}$ are known as black box methods. These use different software and hardware power ${ }_{9}$ ${ }_{10}$ meters to observe performance and resource usage. On the other hand, white box methods ${ }_{10}$ ${ }_{11}$ run a proxy program inside the VE to collect resource utilization data. However, these ${ }_{11}$ ${ }_{12}$ proxies can impact measurements. This is not considered in many studies.

12 13 The survey in [187] found that black box methods using software meters and hardware ${ }_{13}$ ${ }_{14}$ counters are more reliable. Most software meters developed are used to monitor processor ${ }_{14}$ ${ }_{15}$ computing resources and network traffic which later help in developing the power model $_{15}$ ${ }_{16}$ for virtual entities [105]. Since these meters run on the object of study they impose some ${ }_{16}$ ${ }_{17}$ overhead on the system; hence, their own resource consumption must also be considered ${ }_{17}$ ${ }_{18}[116]$. Different software tools are designed to track and log microarchitectural registers and ${ }_{18}$ ${ }_{19}$ counters, to gain knowledge of processor resource consumption. Tools including OProfile ${ }_{19}$ ${ }_{20}$ [188], PERF (ex - Performance Counters for Linux) and sysstat [189], are some examples.20 ${ }_{21}$ They collect data about different processor resources such as cpu usage, cache misses, ${ }_{21}$ ${ }_{22}$ memory access, storage and other performance metrics. Several power models have been ${ }_{22}$ ${ }_{23}$ developed using these tools, with some accounting for the effect of multi-core processors ${ }_{23}$ ${ }_{24}$ in their model.

25 Considering available literature, Linux perf [190] is a widely-used performance-25 26monitoring tool; this latter observation is confirmed in [191]. 26

27 It is integrated in the kernel, thus providing deep insight of system behavior. Fig. $27^{27}$ ${ }^{28}$ shows a frequency analysis of occurrence of different tools found in research studies that ${ }^{28}$ ${ }^{29}$ are part of this survey. PERF is the most widely used (tallest bar in the graph), followed by ${ }^{29}$ ${ }^{30}$ Oprofile.

31 A new energy and performance monitoring tool 'Containergy' is developed in [90] using ${ }^{31}$ ${ }^{32}$ the concept of software containers, that being light weighted incur less overhead. It uses ${ }^{32}$ ${ }^{33}$ the software containers, control groups, hardware performance counters and DVFS to as- ${ }^{33}$ 


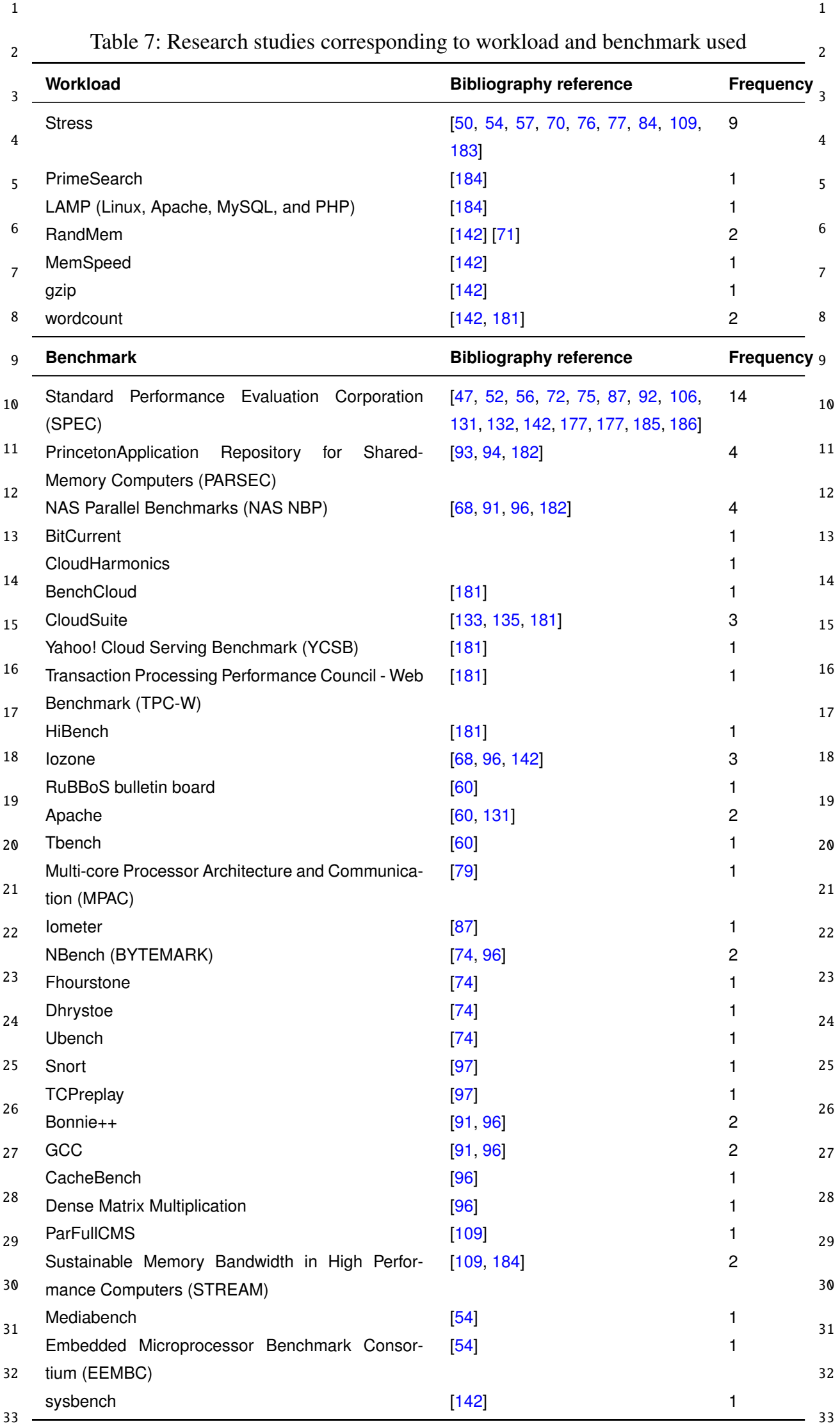


${ }^{1}$ sess the running applications. The effectiveness of the tool is tested using high computing ${ }^{1}$

${ }^{2}$ applications such as machine learning for image classification and video transcoding. $\quad 2$

3 Another tool for process-level power monitoring of virtual entities is developed in [192], ${ }^{3}$

${ }^{4}$ known as 'BitWatts'. This middleware tool accesses the hardware level RAPL counter for ${ }^{4}$

${ }^{5}$ each VM and its running processes. These collected counter values are then transmitted to ${ }^{5}$

${ }^{6}$ respective VMs via the fast communication channel VirtioSerial. This fast channel com- ${ }^{6}$

${ }^{7}$ munication reduces the synchronization error and makes the tool useful for real time power ${ }^{7}$

${ }^{8}$ monitoring of VM processes.

9 Moreover, algorithms and languages chosen for designing a software meter also have sig- ${ }^{9}$

${ }^{10}$ nificant impact on power consumption [183]. This study finds that recursive algorithms are ${ }^{10}$

${ }^{11}$ more energy efficient than the iterative ones. Furthermore, a test program was implemented ${ }^{11}$

${ }^{12} \mathrm{using}$ various programming languages. Implementations of tools in $\mathrm{C}$ and $\mathrm{C}++$ are the most ${ }^{12}$

${ }^{13}$ energy efficient. Implementations in other languages consumed significantly more energy. ${ }^{13}$

${ }^{14}$ Perl consumes the most, followed by OCaml, Python and Prolog. For comparison's sake: ${ }^{14}$

${ }^{15}$ the Perl implementation consumed $25516 \mathrm{~J}$ in this study, while the most energy-efficient ${ }^{15}$

${ }^{16}$ implementation $(\mathrm{C}++)$ consumed just $53 \mathrm{~J}(\mathrm{C}$ implementation: $54.5 \mathrm{~J})$.

17 Notwithstanding improvements in software power meters, none is as precise as exter- ${ }^{17}$

${ }^{18}$ nal hardware meters (e.g. Raritan Dominion PX-5367: error+/-1\%, precision $0.1 \mathrm{~W}$ ). $\mathrm{Ev}_{-}{ }^{18}$

${ }^{19}$ idently, these meters cannot be applied directly to measurement of virtual entities' power ${ }^{19}$

${ }^{20}$ consumption and cannot be used to make quick energy-aware decisions in scheduling, etc. ${ }^{20}$

${ }^{21}$ On the other hand, they can be used to validate power models. Hence, whatever power ${ }^{21}$

${ }^{22}$ model is developed, validation using external power measurements as the ground truth is ${ }^{22}$

${ }^{23}$ important. 23

$24 \quad 24$

${ }_{25} 7.2$ A brief methodology: observations on formal methods 25

${ }_{26}$ We refer to methodology here in the classical sense: a study of alternative methods used ${ }_{26}$ ${ }_{27}$ for modeling, with the intention of picking the method most suited to the task at hand. $A_{27}$ ${ }_{28}$ cross-section through modeling methods found in RUs is presented first (section 7.2.1) ${ }_{28}$ ${ }_{29}$ followed by our recommendations on model selection(section 7.2.2). 29

${ }^{30}$ 7.2.1 A cross-section of modeling methods detected in RUs

${ }^{31}$ The most common methods used to develop power models are linear models [70-72, 74, ${ }^{31}$

$\left.{ }^{32} 75,77,187\right]$. We have confirmed this in the statistics we have compiled (Fig. 26) from ${ }^{32}$

${ }^{33}$ research studies included in this survey: linear models are far more numerous than the rest. ${ }^{33}$ 


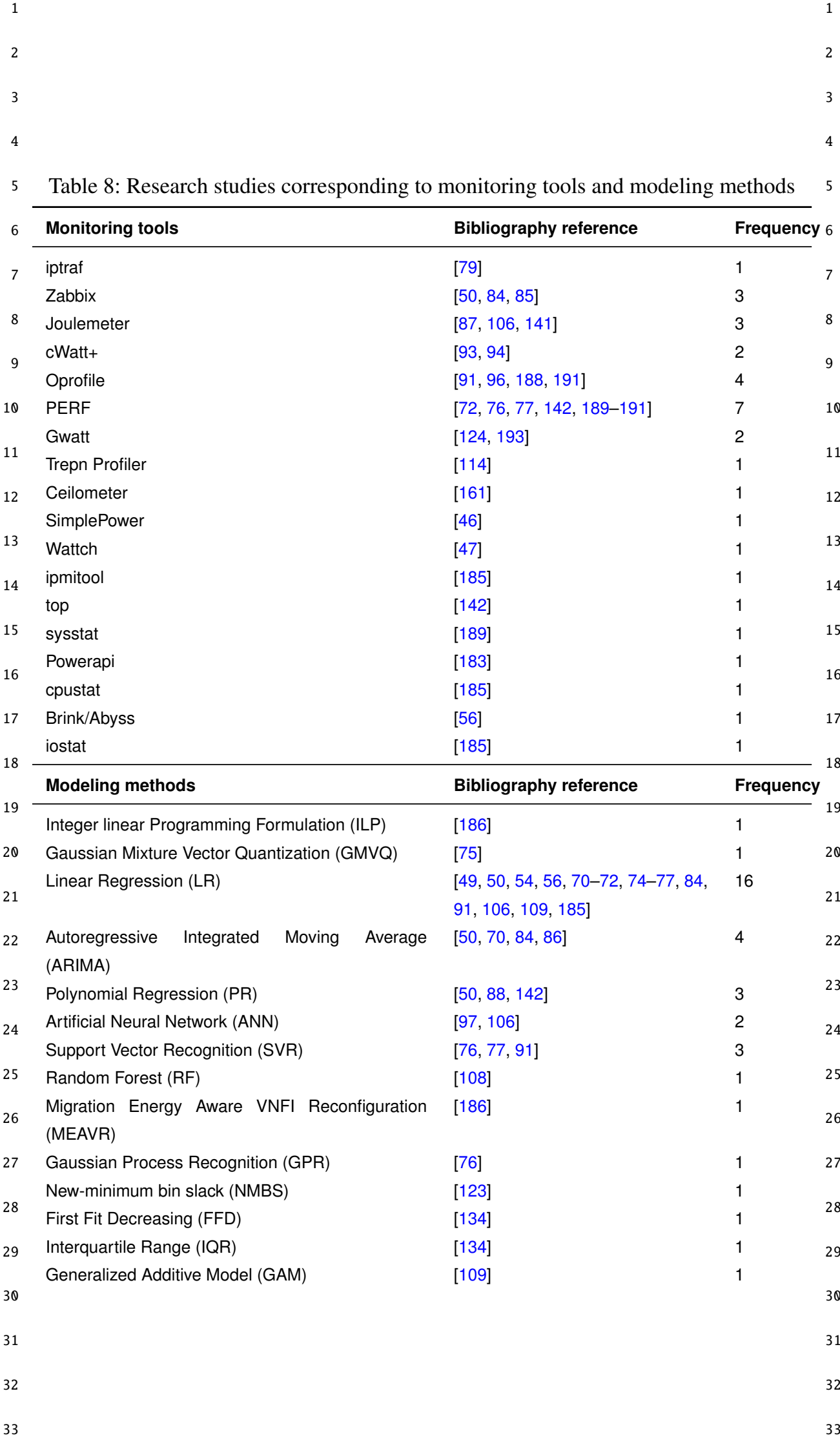




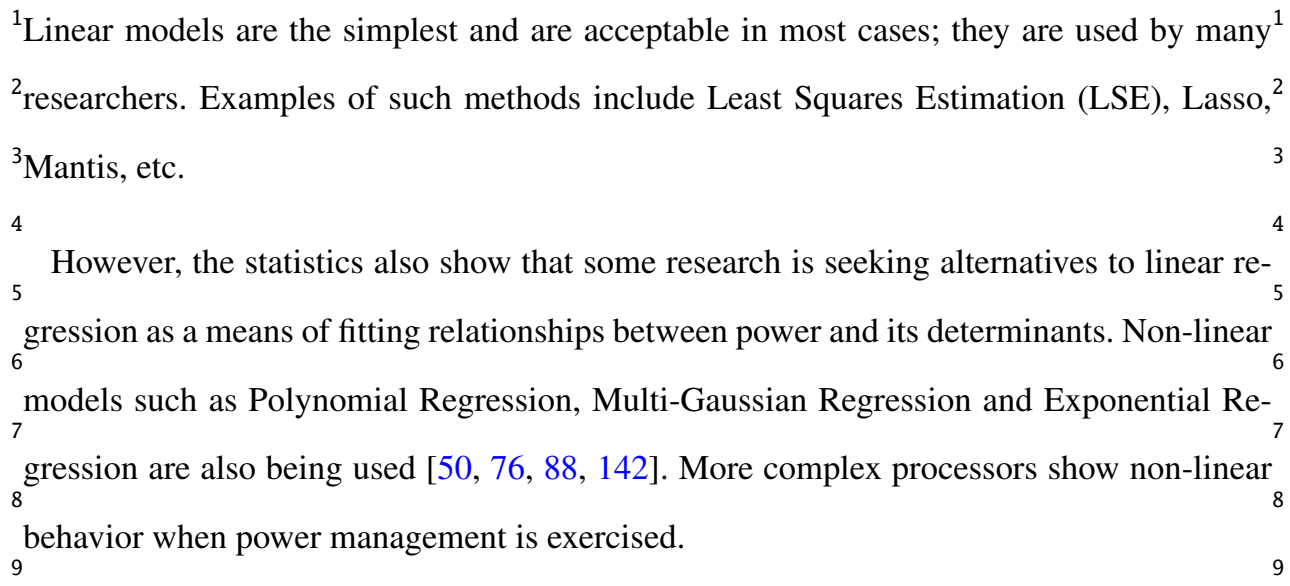

10 The following studies used different modeling methods to improve precision of server 10 11power models.

12 Elman Neural Network. The dynamic traffic behavior in cloud computing and data- ${ }^{12}$ centers causes heterogeneity in the network, which therefore cannot rely on a power ${ }^{13}$ model based on a particular workload. This requires the use of reinforcement learn- ${ }^{14}$ ing for continuous improvement of the model with the changing computing and ${ }^{15}$ networking environment. One approach uses an Elman Neural Network (ENN) to ${ }^{16}$ estimate the power consumed by servers in the cloud [106]. The ENN consists of ${ }^{17}$ input, output and state (instead of hidden) layers, where the state layer is a com- ${ }^{18}$ bination of several hidden layers with local feedback within these hidden layers. This local feedback helps the model to learn the temporal pattern of the dataset for ${ }^{20}$ the specified time interval, which is neglected in other regression methods. Server ${ }^{21}$ resources such as CPU utilization, memory and disk usage, and I/O request rate ${ }^{22}$ are fed as inputs to model the power consumed. Two hyper-parameters - number ${ }^{23}$ of neurons in the state layer and setback time of Back Propagation Through Time ${ }^{24}$ (BPTT) - are optimized for training. When this model was used to estimate power ${ }^{25}$ consumption for two different servers, it showed better accuracy than other exist- ${ }^{26}$ ing Artificial Neural Networks (ANN) and regression models. In [42], application ${ }^{27}$ of artificial neural network (ANN) for power modeling shows enhanced accuracy. ${ }^{28}$ Neural networks with one and two hidden layers are used. Weights and biases to ${ }^{29}$ the neurons are assigned uniformly through Nguyen-Widrow algorithm, and training 30 is performed using Levenberg-Marquardt algorithm which corrects the model based ${ }^{31}$ on mean squared error. To reduce the complexity and computation, the number of variables is further reduced using the forward selection method. 
12 Generalized Additive Models. It is also evident from the literature that servers with ${ }^{1}$ different configurations and multi-core functionality may possess linear or non-linear ${ }^{2}$ behavior in their power consumption depending on the offered load. Hence, differ- ${ }^{3}$ ent studies suggest both linear and non-linear regression methods to develop a power ${ }^{4}$ model for the server. One such study used Simple Linear Regression and Generalized ${ }^{5}$ Additive Models (GAM) to estimate the power consumption of the server under dif- $^{6}$ ferent load conditions [109]. RAPL counters were used to collect the training data. ${ }^{7}$ Later, the accuracy of power estimation for different servers was compared using ${ }^{8}$ an external power meter as ground truth. The model was developed for memory- ${ }^{9}$ intensive applications, using the memory statistics and power consumption as the ${ }^{10}$ predicted and response variable, respectively. Since the power profile of the server ${ }^{11}$ had some non-linearity, GAM predicted power of the server more precisely in the ${ }^{12}$ non-linear region than the linear model.

3 Various other machine-learning-based models. It is challenging to predict real-time ${ }^{14}$ (runtime), optimal, task scheduling with DVFS and Dynamic Power Management ${ }^{15}$ (DPM) enabled. Hence, machine learning based models are preferred, as they can be ${ }^{16}$ trained and adapted. Ordinary Least Squares (OLS), Support Vector Machine (SVM), ${ }^{17}$ ANNs, Naive Bayes, Multinomial Logistic Regression (MLR) are some of the many ${ }^{18}$ supervised learning methods that have been used in different environments to opti- ${ }^{19}$ mize runtime scheduling [194]. Considering the dynamic behavior of servers, differ- $^{20}$ ent reinforcement learning (RL) methods are also being used. Reinforcement learn- ${ }^{21}$ ing algorithms such as Q-learning, On-line distributed, Modular Q-learning, Back ${ }^{22}$ Propagation Neural Networks (BPNN) and Multi-Level RL (MLRL) are mostly used ${ }^{23}$ to minimize the energy consumption of the server while continuously improving the ${ }^{24}$ model [194]. These reinforcement models have been found effective in power man- ${ }^{25}$ agement of datacenters through consolidation and optimal VM allocation. $\quad 26$

4 A new Locally Corrected Multiple Linear Regression (LC-MLR) power model is ${ }^{27}$ proposed in [195], which is an extension to an existing MLR method with additional ${ }^{28}$ error correction. The error correction term is dependent on a linear function of each ${ }^{29}$ resource utilization, obtained using the slope and intercept of the selected resource ${ }^{30}$ interval. The resource interval is the interval between two consecutive points in the ${ }^{31}$ dataset which is selected by locating the particular consumed resource at any in- ${ }^{32}$ stance. In another study [187], researchers apply the tree regression method on the $\mathrm{e}^{33}$ 

collected datasets which is further improved by feeding new data. The basic idea ${ }^{1}$ is to create a tree by dividing the dataset into different partitions until there is no ${ }^{2}$ proper feature found in a newly created partition. Each partition has a set of features ${ }^{3}$ (chosen metric such as CPU utilization, LLC cache miss, etc.) and its corresponding ${ }^{4}$ value. This tree regression model is further optimized through tree pruning, where ${ }^{5}$ the tree model is tested against new datasets to merge some branches and leaf nodes, ${ }^{6}$ resulting in enhanced accuracy of the model.

7

${ }_{11}$ Architecture, system deployment, and workload patterns in computing environments are ${ }_{11}$ ${ }_{12}$ so diverse that no one modeling approach and method can be a universal solution for all ${ }_{12}$ ${ }_{13}$ circumstances. However, for the selection of a model, diagnostic measures are needed to ${ }_{13}$ ${ }_{14}$ assess its reliability.

15 The basic rule of thumb is to select the simplest model and only move to a more complex 15 $160 n e$ if the desired accuracy is not met. The dataset itself contains information such as mul-16 17ticollinearity, number of outliers, size of dataset, non-linearity, etc. that points towards the17 18right choice. The nature of data points, that is whether the input variables are independent 18 19of each other or not, is one important factor.

20 Analyzing the relationship between input and output variables is also useful to find $a^{20}$ ${ }^{21}$ appropriate method. If the relationship between the two is continuous and linear, simple ${ }^{21}$

${ }^{22}$ linear models could be effective.

23 The size of the dataset also plays a role. For some complex models, computation time ${ }^{23}$ ${ }^{24}$ can increase exponentially. Hence, for big data analysis, clustering, classification algo- ${ }^{24}$ ${ }^{25}$ rithms, frequent pattern mining are some techniques to divide the dataset into smaller sets ${ }^{25}$ ${ }^{26}$ and make it feasible for data analysis [196]. Moreover, big data require traditional ma- ${ }^{26}$ ${ }^{27}$ chine learning algorithms to be modified so they can work in parallel computing environ- ${ }^{27}$ ${ }^{28}$ ments. Machine learning algorithms generally used for big data analysis are evolutionary ${ }^{28}$ ${ }^{29}$ algorithms, regression trees, and neural networks. Among these, evolutionary algorithms ${ }^{29}$ ${ }^{30}$ like genetic algorithm, swarm intelligence, and ant clustering are considered more robust ${ }^{30}$ ${ }^{31}$ as their sub-populations can run on parallel computing resources. Hence, these modeling ${ }^{31}$ ${ }^{32}$ methods reduce computation time when run on a GPU, instead of a CPU, on a parallel ${ }^{32}$ 33 33 computing platform. 
1 The ratio of size of the dataset and number of input features is also important to assess ${ }^{1}$

${ }^{2}$ before moving forward. Studies suggested that a single increment in the number of input ${ }^{2}$ ${ }^{3}$ features might lead to an exponential growth in the dataset [197, 198]. With multiple in- ${ }^{3}$ ${ }^{4}$ put features, the dataset must be big enough to avoid the drawback of a form of 'curse of ${ }^{4}$ ${ }^{5}$ dimensionality' that may occur in ML, where increasing dimensions of the feature space ${ }^{5}$ ${ }^{6}$ increase the processing power for data analysis, along with the amount of training data to ${ }^{6}$ ${ }^{7}$ reach a given model accuracy. Other options could be to reduce the number of features ${ }^{7}$ ${ }^{8}$ based on statistical information such as Principal Component Analysis (PCA), probabil- ${ }^{8}$ ${ }^{9}$ ity value (p-value), correlation, stepwise regression, and vector quantization and mixture ${ }^{9}$ ${ }^{10}$ models [198-200].

11 When the relationship among input and output variables is far more complex to identify ${ }^{11}$

${ }^{12}$ using collected data points, non-parametric methods could be useful. Instead of learning ${ }^{12}$

${ }^{13}$ patterns of given data points, these models generate different distributions to extract more ${ }^{13}$

${ }^{14}$ useful information. An example of one such method is Gaussian Process Regression, that ${ }^{14}$

${ }^{15}$ creates the Gaussian distribution for the set of selected data points.

16 We thus observed that no one method is perfect for modeling in all cases. Model accuracy ${ }^{16}$

${ }^{17}$ depends on the right choice of modeling method, via thorough assessment of raw data and ${ }^{17}$

${ }^{18}$ post processing methods available. 18

19

20 Abbreviations 20

21 ACPI Advanced Configuration and Power Interface

22 AF_XDP Address Family eXpress Data Path 22

23 Al $\quad$ Artificial Intelligence 23

24 AR Adaptive Rate 24

BMAP Batch Markov Arrival Process 25

BSS Business Support System 25

26 CAGR Compound Annual Growth Rate 26

CNF Containerized Network Function 27

28 COTS Commodity-Off-The-Shelf 28

CPU Central Processing Unit

30 CRM Customer Relationship Management 30

DAG Directed Acyclic Graph

31 DDIO Data Direct Input/Output 31

DPDK Data Plane Development Kit 32

33 EPC Evolved Packet Core 33 
1 ETSI European Telecommunications Standards Institute

2 FLPA Functional-Level Power Analysis 2

GAM Generalized Additive Model

3 GBP Greedy Bin Packing 3

4 GPU Graphics Processing Unit 4

$\begin{array}{ll}\text { HEVC } & \text { High Efficiency Video Coding } \\ \text { ICT } & \text { Information and Communication Technology }\end{array}$

6 IDS Intrusion Detection System 6

IN Intermediate Node

$\begin{array}{lll}7 & \mathrm{Pr} & \text { Infrastructure Provider }\end{array}$

ISP Internet Service Provider

8 KVM Kernel Virtual Machine 8

9 L2 Layer 2 (or Level 2) 9

$\begin{array}{lll}\text { L3 } & \text { Level } 3 & 10 \\ \text { LLC } & \text { Last Level Cache }\end{array}$

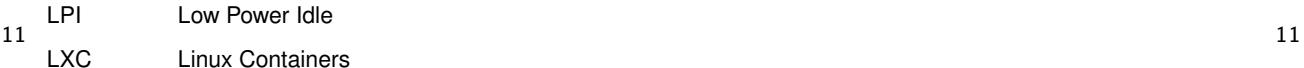

12 MANO Management and Orchestration 12

MCF Multi-Commodity Flow

13 MEC Multi-access edge computing 13

14 MIPS Millions of instructions per second 14

ML Machine Learning

15 MOPTS Million operations per time slot 15

16 MOS Metal-Oxide-Semiconductor 16

$\begin{array}{lll}\text { NANOG } & \text { North American Network Operators' Group } & 17 \\ \text { NFV } & \text { Network Function Virtualization } & 17\end{array}$

18 NIC Network Interface Card 18

$\begin{array}{ll}\text { NUMA } & \text { Non-Uniform Memory Architecture } \\ \text { O\&M } & \text { Operation, administration, maintenance and management }\end{array}$

OPEX Operational Expenditure

20 OS Operating System 20

21 OSS Operational Support System 21

OvS Open Virtual Switch

22 PAD Problem-Approach-Development 22

23 PDU Power Distribution Unit 23

24 PNF Physical Network Function 24

$\begin{array}{lll}\text { PoP } & \text { Point of Presence } & 25\end{array}$

26 PTNO Public Telecommunications Network Operator 26

$\begin{array}{lll}\text { QAT } & \text { Quick Assist Technology } & 27\end{array}$

28 RES Renewable Energy Sources 28

$\begin{array}{lll}\text { RRH } & \text { Remote Radio Head } & 29 \\ \text { SBA } & \text { Service-Based Architecture }\end{array}$

30 SDN Software-Defined Networking 30

SGW Serving Gateway 31

31 SLA Service-Level Agreement 31

32 SoC System-on-Chip 32

33 SPEC Standard Performance Evaluation Corporation 33 
1 SPOF Single Point of Failure

2 SRIA Strategic Research and Innovation Agenda 2

SR-IOV Single-Root Input/Output Virtualization

3 SVM Support Vector Machine 3

4 TCO Total Cost of Ownership 4

$\begin{array}{ll}\text { TCP } & \text { Transmission Control Protocol } \\ \text { TOE } & \text { TCP Offload Engine }\end{array}$

$\begin{array}{lll}\text { vBBU Virtual Baseband Unit } & 6\end{array}$

$\begin{array}{lll}7 \text { VDU Virtual Deployment Unit } & 7\end{array}$

VE Virtual Entity

8 VM Virtual Machine $\quad 8$

9 VNF Virtual Network Function 9

VNFD Virtual Network Function Descriptor

$10 \mathrm{VNI}$ Virtual networking index 10

11 WSC Warehouse Scale Computing

11

$12-12$

13

Availability of data and materials

14The dataset supporting the conclusions of this article is available in the PowerMeasurementAndModelingRawData 14

repository, where we present all triads (Triads.xlsx) mined from the corpus. This is the data which was digested to produce

15 the statistics shown in section 3 . The repository also carries a second file (NodeAttributes.xlsx), which tabulates the

16 number and label attributes of the nodes. 16

$\begin{array}{ll}17 \text { Competing interests } & 17\end{array}$

The authors declare that they have no competing interests.

Authors' contributions

${ }^{19}$ Etienne-Victor Depasquale contributed to the conceptualization of this paper, the methodology, formal analysis, 19

20 investigation, resources, writing of the original draft, data curation and visualization. 20

Humaira Abdul Salam contributed to the conceptualization of this paper, investigation, resources, data curation, writing of

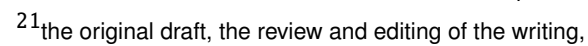

${ }_{22}$ Franco Davoli contributed to the resources, writing of the original draft, the review and editing of the writing and the 22 supervision.

23

Author details

${ }^{241}$ Department of Communications and Computer Engineering, University of Malta, MSD2080 Msida, Malta. ${ }^{2}$ High-Energy 24

25 Physics Research (FH) - IT, Deutsches Elektronen-Synchrotron (DESY), 22607 Hamburg, Germany. ${ }^{3}$ Department of 25

Electrical, Electronic and Telecommunications Engineering and Naval Architecture (DITEN), University of Genoa, 16145

26Genoa, Italy.

27References

1. Bolla, R., Bruschi, R., Davoli, F., Cucchietti, F.: Energy Efficiency in the Future Internet: A Survey of Existing Approaches and Trends in Energy-Aware Fixed Network Infrastructures. IEEE Communications Surveys Tutorials

29 13(2), 223-244 (2011). doi:10.1109/SURV.2011.071410.00073

2. Idzikowski, F., Chiaraviglio, L., Cianfrani, A., López Vizcaíno, J., Polverini, M., Ye, Y.: A Survey on Energy-Aware Design and Operation of Core Networks. IEEE Communications Surveys Tutorials 18(2), 1453-1499 (2016). doi:10.1109/COMST.2015.2507789

3. Maaloul, R., Chaari, L., Cousin, B.: Energy saving in carrier-grade networks: A survey. Computer Standards \&

32 Interfaces 55, 8-26 (2018). doi:10.1016/j.csi.2017.04.001. Accessed 2020-07-26

4. Delforge, P., Whitney, J.: Data center efficiency assessment - scaling up energy efficiency across the data center industry: evaluating key drivers and barriers. Technical Report IP:14.08-A, Natural Resources Defense Council 
3 5. Cisco visual networking index: Forecast and methodology, 2014-2019. Technical report, Cisco Systems, San Jose, 3 CA (2015)

6. Cisco visual networking index: Forecast and methodoolgy, 2015-2020. Technical report, Cisco Systems, San Jose,

7. Cisco visual networking index: Forecast and methodology, 2016-2021. Technical report, Cisco Systems, San Jose, CA (2017)

7 8. Cisco Visual Networking Index: Forecast and Trends, 2017-2022. Technical report, Cisco Systems, San Jose, CA 7 (2019)

8 9. Ishii, K., Kurumida, J., Sato, K.-i., Kudoh, T., Namiki, S.: Unifying Top-Down and Bottom-Up Approaches to Evaluate 8

$9 \quad$ Network Energy Consumption. Journal of Lightwave Technology 33(21), 4395-4405 (2015). doi:10.1109/JLT.2015.2469145

10 10. Metro network traffic growth: an architecture impact study. Technical report, Alcatel-Lucent (2013)

11. Norton, W.B.: The 2014 Internet Peering Playbook: Connecting to the Core of The Internet. DrPeering Press, ??? (2014)

12 12. Global Internet Phenomena Report. Technical report, Sandvine (2013). https:

//www.sandvine.com/hubfs/downloads/archive/2013-2h-global-internet-phenomena-report.pdf Accessed 2020-07-04

14 13. Global Internet Phenomena - Africa, Middle East \& North America. Technical report (December 2015). 14 https://www. sandvine.com/hubfs/downloads/archive/

15 2015-global-internet-phenomena-report-africa-middle-east-and-north-america.pdf Accessed 2020-07-04

14. Global Internet Phenomena Report. Technical report, Sandvine (September 2019)

17 15. Lee, U., Rimac, I., Hilt, V.: Greening the internet with content-centric networking. In: Proceedings of the 1 st 17 International Conference on Energy-Efficient Computing And Networking. e-Energy '10, pp. 179-182. Association for Computing Machinery, Passau, Germany (2010). doi:10.1145/1791314.1791342.

19 https://doi.org/10.1145/1791314.1791342 Accessed 2020-07-04

16. Guan, K., Atkinson, G., Kilper, D.C., Gulsen, E.: On the Energy Efficiency of Content Delivery Architectures. In: 2011 IEEE International Conference on Communications Workshops (ICC), pp. 1-6 (2011).

21 doi:10.1109/iccw.2011.5963557

17. Choi, N., Guan, K., Kilper, D.C., Atkinson, G.: In-network caching effect on optimal energy consumption in

22 content-centric networking. In: 2012 IEEE International Conference on Communications (ICC), pp. 2889-2894 22

(2012). doi:10.1109/ICC.2012.6364320

18. Osman, N.I., El-Gorashi, T., Elmirghani, J.M.H.: Reduction of energy consumption of Video-on-Demand services

24 using cache size optimization. In: 2011 Eighth International Conference on Wireless and Optical Communications 24 Networks, pp. 1-5 (2011). doi:10.1109/WOCN.2011.5872923

19. Llorca, J., Tulino, A.M., Guan, K., Esteban, J., Varvello, M., Choi, N., Kilper, D.C.: Dynamic in-network caching for

26 energy efficient content delivery. In: 2013 Proceedings IEEE INFOCOM, pp. 245-249 (2013). 26 doi:10.1109/INFCOM.2013.6566772

27 20. Mandal, U., Chowdhury, P., Lange, C., Gladisch, A., Mukherjee, B.: Energy-efficient networking for content 27

28 distribution over telecom network infrastructure. Optical Switching and Networking 10(4), 393-405 (2013). 28 doi:10.1016/j.osn.2013.06.003. Accessed 2020-07-04

29 21. Modrzejewski, R., Chiaraviglio, L., Tahiri, I., Giroire, F., Le Rouzic, E., Bonetto, E., Musumeci, F., Gonzalez, R., 29 Guerrero, C.: Energy efficient content distribution in an ISP network. In: 2013 IEEE Global Communications Conference (GLOBECOM), pp. 2859-2865 (2013). doi:10.1109/GLOCOM.2013.6831508

31 22. Abji, N., Tizghadam, A., Leon-Garcia, A.: Energy efficient content delivery in service provider networks with content 31 caching. In: 2015 IEEE Online Conference on Green Communications (OnlineGreenComm), pp. 23-29 (2015). doi:10.1109/OnlineGreenCom.2015.7387374

33 23. Savi, M., Ayoub, O., Musumeci, F., Li, Z., Verticale, G., Tornatore, M.: Energy-efficient caching for Video-on-Demand 33 
24. Jayasundara, C., Nirmalathas, A., Wong, E., Chan, C.: Improving Energy Efficiency of Video on Demand Services. IEEE/OSA Journal of Optical Communications and Networking 3(11), 870-880 (2011). doi:10.1364/JOCN.3.000870 3

4 25. Fratini, R., Savi, M., Verticale, G., Tornatore, M.: Using replicated video servers for VoD traffic offloading in integrated 4 metro/access networks. In: 2014 IEEE International Conference on Communications (ICC), pp. 3438-3443 (2014).

5 doi:10.1109/ICC.2014.6883853

6. Pascale, E.D., Payne, D.B., Ruffini, M.: Bandwidth and energy savings of locality-aware P2P Content Distribution in next-generation PONs. In: 2012 16th International Conference on Optical Network Design and Modelling (ONDM),

7 pp. 1-6 (2012). doi:10.1109/ONDM.2012.6210215

27. Y.3001 : Future networks: Objectives and design goals (2011). Publisher: ITU-T

8 28. Pervasive Mobile Virtual Services. Technical report, Expert Advisory Group of the European Technology Platform

9 Networld 2020 (July 2016). https://www. networld2020.eu/wp-content/uploads/2014/02/SRIA_final.pdf 9

29. Bouchat, C., Paul, M., Allan, D., Dalle, G.: 5 G Wireless Wireline Convergence Atchitecture. Technical Report TR-470,

10 Broadband Forum (August 2020). https://www. broadband-forum. org/technical/download/TR-470.pdf 10

Accessed 2020-10-03

30. Environmental Engineering (EE); Green Abstraction Layer (GAL); Power management capabilities of the future

12 energy telecommunication fixed network nodes. ETSI Standard ETSI ES 203237 V1.1.1, European 12 Telecommunications Standards Institute (March 2014)

13 31. Bolla, R., Bruschi, R., Davoli, F., Lombardo, C., Pajo, J.F., Sanchez, O.R.: The dark side of network functions

14 virtualization: A perspective on the technological sustainability. In: IEEE International Conference on Communications (ICC), pp. 1-7 (2017). doi:10.1109/ICC.2017.7997129

15 32. Network Functions Virtualisation (NFV); Use Cases. Technical Report RGR/NFV-001ed121, ETSI (May 2017)

16 33. Shi, W.: Making sense of the telco cloud. Technical report, Telecoms.com (May 2020).

https://telecoms . com/opinion/making-sense-of-the-telco-cloud/ Accessed 2020-06-27

17 34. Edge computing and deployment strategies for communication service providers. Technical Report

GFMC-20:000097, Ericsson (February 2020). https://www . ericsson. com/en/reports-and-papers/

35. Crawshaw, J.: Deconstructing the Telco Cloud. Light Reading (2019). Accessed 2020-07-25

20 36. Ayoubi, S.E.E., Jeux, S., Marache, F., Pujol, F., Fallgren, M., Spapis, P., Yang, C., Widaa, A., Markendahl, J., 20

21 Ghanbari, A., Ruismaki, R., Uusitalo, M., Koenig, W., Trogolo, A., Korthals, I., Rosowski, T., Zimmermann, G., 21 Campoy, L., Monserrat, J., Martin-Sacristan, D., Schotten, H., Qi, Y., Singh, S., Agyapong, P.: Refined scenarios and requirements, consolidated use cases, and qualitative techno-economic feasibility assessment. Technical Report 22 D1.1 (January 2016). https://metis-ii.5g-ppp.eu/wp-content/uploads/deliverables/METIS-II_D1.1_v1.0.pdf Accessed

37. Muthurajan, J.: Why Use Cloud-Native Containerized Network Functions (CNFs)? White paper, Intel.

26 why-containers-and-cloud-native-functions-paper .html Accessed 2020-07-25

38. Vettor, R., Ardalis Smith, S.: Architecting Cloud Native .NET Applications for Azure (2020)

27 39. Logan, M.: CNCF F Technical Oversight Committee (TOOC). GitHub (2021)

41. Dayarathna, M., Wen, Y., Fan, R.: Data Center Energy Consumption Modeling: A Survey. IEEE Communications

29 Surveys Tutorials 18(1), 732-794 (2016). doi:10.1109/COMST.2015.2481183

30 42. Costa, G.D., Pierson, J.-M., Fontoura-Cupertino, L.: Effectiveness of Neural Networks for Power Modeling for Cloud and HPC: It's Worth It! ACM Transactions on Modeling and Performance Evaluation of Computing Systems 5(3),

31 1-36 (2020). doi:10.1145/3388322. Accessed 2020-10-25

43. Ismail, L., Materwala, H.: Computing Server Power Modeling in a Data Center: Survey, Taxonomy, and Performance Evaluation. ACM Computing Surveys 53(3), 1-34 (2020). doi:10.1145/3390605. Accessed 2020-07-07 32 
45. Depasquale, E.-V., Salam, H.A., Davoli, F.: PAD: a graphical and numerical enhancement of structural coding to facilitate thematic analysis of a literature corpus. arXiv:2107.13983 [cs] (2021). arXiv: 2107.13983. Accessed 3 2021-07-31 estimation tool. In: 37th Conference on Design Automation - DAC '00, pp. 340-345. ACM Press, Los Angeles, California, United States (2000). doi:10.1145/337292.337436. http://portal.acm.org/citation.cfm?doid=337292.337436 Accessed 2019-12-18

7 47. Brooks, D., Tiwari, V., Martonosi, M.: Wattch: a framework for architectural-level power analysis and optimizations. In:7 27th International Symposium on Computer Architecture (IEEE Cat. No.RS00201), pp. 83-94 (2000)

8 48. Fan, X., Weber, W.-D., Barroso, L.A.: Power provisioning for a warehouse-sized computer. ACM SIGARCH Computer ${ }^{8}$

9 Architecture News 35(2), 13-23 (2007). doi:10.1145/1273440.1250665. Accessed 2020-10-05

49. Vasan, A., Sivasubramaniam, A., Shimpi, V., Sivabalan, T., Subbiah, R.: Worth their watts? - an empirical study of

10 datacenter servers. In: HPCA - 162010 The Sixteenth International Symposium on High-Performance Computer Architecture, pp. 1-10 (2010). doi:10.1109/HPCA.2010.5463056

50. Aldossary, M., Alzamil, I., Djemame, K.: Towards Virtual Machine Energy-Aware Cost Prediction in Clouds. In: Pham,

12 C., Altmann, J., Bañares, J.A. (eds.) Economics of Grids, Clouds, Systems, and Services. Lecture Notes in Computer12 Science, pp. 119-131. Springer, Cham (2017). doi:10.1007/978-3-319-68066-8_10

13 51. Bellosa, F.: The benefits of event: driven energy accounting in power-sensitive systems. In: Proceedings of the 9th

14 Workshop on ACM SIGOPS European Workshop Beyond the PC: New Challenges for the Operating System - EW 9 , 14 p. 37. ACM Press, Kolding, Denmark (2000). doi:10.1145/566726.566736

15 52. Isci, C., Martonosi, M.: Runtime power monitoring in high-end processors: methodology and empirical data. In: 15

16 Proceedings. 36th Annual IEEE/ACM International Symposium on Microarchitecture, 2003. MICRO-36., pp. 93-104 16 (2003). doi:10.1109/MICRO.2003.1253186

17 53. Laurent, J., Julien, N., Senn, E., Martin, E.: Functional level power analysis: an efficient approach for modeling the 17 power consumption of complex processors. In: Automation and Test in Europe Conference and Exhibition Proceedings Design, vol. 1, pp. 666-6671 (2004). doi:10.1109/DATE.2004.1268921

19 54. Rethinagiri, S.K., Palomar, O., Moreno, J.A., Unsal, O., Cristal, A.: VPM: Virtual power meter tool for low-power 19 many-core/heterogeneous data center prototypes. In: 2015 33rd IEEE International Conference on Computer Design (ICCD), pp. 651-658 (2015). doi:10.1109/ICCD.2015.7357177

21 55. Bertran, R., Gonzàlez, M., Martorell, X., Navarro, N., Ayguadé, E.: Counter-based power modeling methods: 21 Top-down vs. bottom-up. The Computer Journal 56(2), 198-213 (2013)

22 56. Bircher, W.L., Valluri, M., Law, J., John, L.K.: Runtime identification of microprocessor energy saving opportunities. 22 In: Proceedings of the 2005 International Symposium on Low Power Electronics And Design., pp. 275-280 (2005). 23 doi:10.1145/1077603.1077668

24 57. Khan, N.: Investigating Energy Efficiency of Physical and Virtual Machines in Cloud Computing. PhD thesis, 24 Univeristy of Oslo (2017). https://www . duo. uio.no/handle/10852/61321 Accessed 2019-11-07

25 58. Chen, Q., Grosso, P., Veldt, K.v.d., Laat, C.d., Hofman, R., Bal, H.: Profiling Energy Consumption of VMs for Green

26 Cloud Computing. In: 2011 IEEE Ninth International Conference on Dependable, Autonomic and Secure Computing, 26 pp. 768-775. IEEE, Sydney, Australia (2011). doi:10.1109/DASC.2011.131.

27 http://ieeexplore.ieee.org/document/6118905/ Accessed 2021-02-18

28 59. Morabito, R.: Power Consumption of Virtualization Technologies: An Empirical Investigation. In: 2015 IEEE/ACM 8th 28 International Conference on Utility and Cloud Computing (UCC), pp. 522-527 (2015). doi:10.1109/UCC.2015.93

29 60. Shea, R., Wang, H., Liu, J.: Power consumption of virtual machines with network transactions: Measurement and 29 improvements. In: IEEE INFOCOM 2014 - IEEE Conference on Computer Communications, pp. 1051-1059 (2014). 30 doi:10.1109/INFOCOM.2014.6848035

3161 . Hu, Y., Li, T.: Towards efficient server architecture for virtualized network function deployment: Implications and 31 implementations. In: 2016 49th Annual IEEE/ACM International Symposium on Microarchitecture (MICRO), pp. 1-12 (2016). doi:10.1109/MICRO.2016.7783711

33 62. Xu, Z., Liu, F., Wang, T., Xu, H.: Demystifying the energy efficiency of Network Function Virtualization. In: 2016 
63. Fu, S., Liu, J., Zhu, W.: Multimedia Content Delivery with Network Function Virtualization: The Energy Perspective.

4 64. Li, X., Cheng, W., Zhang, T., Ren, F., Yang, B.: Towards Power Efficient High Performance Packet I/O. IEEE Transactions on Parallel and Distributed Systems 31(4), 981-996 (2020). doi:10.1109/TPDS.2019.2957746

5 65. CNF friendly networking for Telco/Edge Kubernetes platforms (2020). Accessed 2020-09-28

66. Li, G., Zhang, D., Li, Y., Li, K.: Toward energy-efficiency optimization of pktgen-DPDK for green network testbeds. China Communications 15(11), 199-207 (2018). doi:10.1109/CC.2018.8543100

7 67. Intel Ethernet Server Adapter I350: Product Brief. Intel (2021). Accessed 2021-02-09

68. Zhang, Z., Fu, S.: Macropower: A coarse-grain power profiling framework for energy-efficient cloud computing. In: 30th IEEE International Performance Computing and Communications Conference, pp. 1-8 (2011).

9 doi:10.1109/PCCC.2011.6108061

69. KVM (2021). Accessed 2021-03-02

10 70. Aldossary, M., Djemame, K., Alzamil, I., Kostopoulos, A., Dimakis, A., Agiatzidou, E.: Energy-aware cost prediction 10 and pricing of virtual machines in cloud computing environments. Future Generation Computer Systems 93, 442-459 (2019). doi:10.1016/j.future.2018.10.027. Accessed 2020-07-23

12 71. Li, Y., Wang, Y., Yin, B., Guan, L.: An Online Power Metering Model for Cloud Environment. In: 2012 IEEE 11 th 12 International Symposium on Network Computing and Applications, Cambridge, MA, pp. 175-180 (2012).

14 72. Wen, C., Long, X., Yang, Y., Ni, F., Mu, Y.: System Power Model and Virtual Machine Power Metering for Cloud Computing Pricing. In: 2013 Third International Conference on Intelligent System Design and Engineering

15 Applications, pp. 1379-1382 (2013). doi:10.1109/ISDEA.2012.327

16 73. Arroba, P., Risco-Martín, J.L., Zapater, M., Moya, J.M., Ayala, J.L., Olcoz, K.: Server Power Modeling for Run-time 16 Energy Optimization of Cloud Computing Facilities. Energy Procedia 62, 401-410 (2014).

17 doi:10.1016/j.egypro.2014.12.402. Accessed 2019-12-17

74. Wu, W., Lin, W., Peng, Z.: An intelligent power consumption model for virtual machines under CPU-intensive workload in cloud environment. Soft Computing 21(19), 5755-5764 (2017). doi:10.1007/s00500-016-2154-6

19 75. Dhiman, G., Mihic, K., Rosing, T.: A system for online power prediction in virtualized environments using Gaussian 19 mixture models. In: Proceedings of the 47th Design Automation Conference, p. 807. ACM Press, Anaheim, CA (2010). doi:10.1145/1837274.1837478

21 76. Salam, H.A., Davoli, F., Timm-Giel, A.: Improving Prediction Accuracy for Power Consumption in Virtual Environments. In: 2019 29th International Telecommunication Networks and Applications Conference (ITNAC), pp 1-6 (2019). doi:10.1109/ITNAC46935.2019.9077952

33 77. Salam, H.A., Davoli, F., Carrega, A., Timm-Giel, A.: Towards Prediction of Power Consumption of Virtual Machines for Varying Loads. In: 2018 28th International Telecommunication Networks and Applications Conference (ITNAC), pp. 1-6 (2018). doi:10.1109/ATNAC.2018.8615319

Callau-Zori, M., Samoila, L., Orgerie, A.-C., Pierre, G.: An experiment-driven energy consumption model for virtual machine management systems. Sustainable Computing: Informatics and Systems 18, 163-174 (2018).

79. Chinprasertsuk, S., Gertphol, S.: Power model for virtual machine in cloud computing. In: 2014 11th International Joint Conference on Computer Science and Software Engineering (JCSSE), pp. 140-145 (2014). doi:10.1109/JCSSE.2014.6841857

80. Enokido, T., Takizawa, M.: The Extended Power Consumption Model to Perform Computation Type Application

29 Processes on Virtual Machines. In: 2016 10th International Conference on Complex, Intelligent, and Software Intensive Systems (CISIS), pp. 15-22. IEEE, Fukuoka, Japan (2016). doi:10.1109/CISIS.2016.82. http://ieeexplore.ieee.org/document/7791857/ Accessed 2019-12-18 Processes on Virtual Machines. In: 2015 10th International Conference on Broadband and Wireless Computing, Communication and Applications (BWCCA), pp. 275-282 (2015). doi:10.1109/BWCCA.2015.67 
2 83. Enokido, T., Takizawa, M.: Power Consumption and Computation Models of Virtual Machines to Perform Computation 2 Type Application Processes. In: 2015 Ninth International Conference on Complex, Intelligent, and Software Intensive

3 Systems, pp. 126-133 (2015). doi:10.1109/CISIS.2015.18

4 84. Aldossary, M., Djemame, K.: Performance and Energy-based Cost Prediction of Virtual Machines Live Migration in 4 Clouds:. In: Proceedings of the 8th International Conference on Cloud Computing and Services Science, pp.

5 384-391. SCITEPRESS - Science and Technology Publications, Funchal, Madeira, Portugal (2018). 5 doi:10.5220/0006682803840391.

6 http//www.scitepress.org/DigitalLibrary/Link.aspx?doi=10.5220/0006682803840391 Accessed 2020-07-23

7 85. Aldossary, M., Djemame, K.: Energy-based Cost Model of Virtual Machines in a Cloud Environment. In: 2018 Fifth 7 International Symposium on Innovation in Information and Communication Technology (ISIICT), pp. 1-8 (2018).

8 doi:10.1109/ISIICT.2018.8613288

9 86. Alzamil, I., Djemame, K.: Energy Prediction for Cloud Workload Patterns. In: Bañares, J.A., Tserpes, K., Altmann, J. 9 (eds.) Economics of Grids, Clouds, Systems, and Services. Lecture Notes in Computer Science, pp. 160-174

10 Springer, Cham (2017). doi:10.1007/978-3-319-61920-0_12

87. Kansal, A., Zhao, F., Liu, J., Kothari, N., Bhattacharya, A.A.: Virtual machine power metering and provisioning. In: Proceedings of the 1st ACM Symposium on Cloud Computing - SoCC '10, p. 39. ACM Press, Indianapolis, Indiana,

12 USA (2010). doi:10.1145/1807128.1807136. http://portal.acm.org/citation.cfm?doid=1807128.1807136 Accessed 12 2020-07-23

13 88. Waßmann, I., Versick, D., Tavangarian, D.: Energy consumption estimation of virtual machines. In: Proceedings of

14 the 28th Annual ACM Symposium on Applied Computing. SAC '13, pp. 1151-1156. Association for Computing 14 Machinery, Coimbra, Portugal (2013). doi:10.1145/2480362.2480579. https://doi.org/10.1145/2480362.2480579

15 Accessed 2020-07-23

16 89. Gu, C., Shi, P., Shi, S., Huang, H., Jia, X.: A Tree Regression Based Approach for VM Power Metering. IEEE Access 16 3, 1-1 (2015). doi:10.1109/ACCESS.2015.2430276

17 90. Silva-de-Souza, W., Iranfar, A., Bráulio, A., Zapater, M., Xavier-de-Souza, S., Olcoz, K., Atienza, D.: Containergy-A 17 Container-Based Energy and Performance Profiling Tool for Next Generation Workloads. Energies 13(9), 2162 (2020). doi:10.3390/en13092162. Accessed 2020-10-25

19 91. Veni, T., Bhanu, S.M.S.: Prediction Model for Virtual Machine Power Consumption in Cloud Environments. Procedia 19 Computer Science 87, 122-127 (2016). doi:10.1016/j.procs.2016.05.137

20 92. SPEC Benchmarks (2020)

21 93. Phung, J., Young, C.L., Zomaya, A.Y.: Application-Agnostic Power Monitoring in Virtualized Environments. In: 201721 17th IEEE/ACM International Symposium on Cluster, Cloud and Grid Computing (CCGRID), pp. 335-344 (2017).

22 doi:10.1109/CCGRID.2017.100

3 94. Phung, J., Lee, Y.C., Zomaya, A.Y.: Lightweight Power Monitoring Framework for Virtualized Computing Environments. IEEE Transactions on Computers 69(1), 14-25 (2020). doi:10.1109/TC.2019.2936018

24 95. Bolla, R., Bruschi, R., Davoli, F., Pajo, J.F.: A Model-Based Approach Towards Real-Time Analytics in NFV Infrastructures. IEEE Transactions on Green Communications and Networking 4(2), 529-541 (2020).

26 96. Husain Bohra, A.E., Chaudhary, V.: VMeter: Power modelling for virtualized clouds. In: 2010 IEEE International 26 Symposium on Parallel Distributed Processing, Workshops and Phd Forum (IPDPSW), pp. 1-8 (2010).

27 doi:10.1109/IPDPSW.2010.5470907

28 97. Moreira Zorello, L.M., Torres Vieira, M.G., Girani Tejos, R.A., Torres Rojas, M.A., Meirosu, C., Melo de Brito Carvalho, 28 T.C.: Improving Energy Efficiency in NFV Clouds with Machine Learning. In: 2018 IEEE 11th International

29 Conference on Cloud Computing (CLOUD), pp. 710-717 (2018). doi:10.1109/CLOUD.2018.00097 29

30 98. Stoess, J., Lang, C., Bellosa, F.: Energy Management for Hypervisor-Based Virtual Machines. In: USENIX Annual Technical Conference, Santa Clara, CA (2007)

31 99. Virtio - KVM (2020). Accessed 2020-08-27

100. Poll Mode Driver — Data Plane Development Kit 20.11.0-rc0 documentation (2014)

32 101. The Zeek Network Security Monitor. Zeek (2020). Accessed 2020-10-02 
103. Open vSwitch (2020). Accessed 2020-08-27 1

${ }_{2}$ 104. Rizzo, L., Lettieri, G.: VALE, a switched ethernet for virtual machines. In: Proceedings of the 8th International 2 Conference on Emerging Networking Experiments and Technologies. CoNEXT '12, pp. 61-72. Association for

3 Computing Machinery, New York, NY, USA (2012). doi:10.1145/2413176.2413185. 3 https://doi.org/10.1145/2413176.2413185 Accessed 2020-11-18

105. Bruschi, R., Davoli, F., Lago, P., Pajo, J.F.: Joint Power Scaling of Processing Resources and Consolidation of Virtual

$5 \quad$ Network Functions. In: 5th IEEE International Conference on Cloud Networking (Cloudnet), pp. 70-75 (2016). 5 doi:10.1109/CloudNet.2016.20

6 106. Wu, W., Lin, W., He, L., Wu, G., Hsu, C.: A Power Consumption Model for Cloud Servers Based on Elman Neural Network. IEEE Transactions on Cloud Computing, 1-1 (2019). doi:10.1109/TCC.2019.2922379 7

107. Łukasik, R.: Run-to-completion model in Data Plane processing - Nokia Wrocław (2019). Accessed 2020-09-30

8 108. Kai, C., Blesson, V., Peter, K., S, N.D.: Power Modelling for Heterogeneous Cloud-Edge Data Centers. Advances in 8 Parallel Computing 32, 804-813 (2018). doi:10.3233/978-1-61499-843-3-804. Accessed 2019-12-17

109. Khan, K.N., Hirki, M., Niemi, T., Nurminen, J.K., Ou, Z.: RAPL in Action: Experiences in Using RAPL for Power

10 Measurements. ACM Transactions on Modeling and Performance Evaluation of Computing Systems 3(2), 9-1926 10 (2018). doi:10.1145/3177754. Accessed 2020-07-27

11 110. Molnar, I.: Performance Counters for Linux, v8 (2009). Accessed 2020-07-23

12111. Bolla, R., Bruschi, R., Carrega, A., Davoli, F.: Green network technologies and the art of trading-off. In: 2011 IEEE 12 Conference on Computer Communications Workshops (INFOCOM WKSHPS), pp. 301-306 (2011). doi:10.1109/INFCOMW.2011.5928827

14112. TPC-W Homepage (2021). Accessed 2021-02-11

113. Advanced Configuration and Power Interface (ACPI) Specification - ACPI Specification 6.4 documentation (2021). 15 Accessed 2021-03-02

$16{ }^{114 .}$ Keong, C.K., Wei, K.T., Ghani, A.A.A., Sharif, K.Y.: Toward using software metrics as indicator to measure power consumption of mobile application: A case study, pp. 172-177 (2015). doi:10.1109/MySEC.2015.7475216

17115. Li, K.: Optimal configuration of a multicore server processor for managing the power and performance tradeoff. The 17 Journal of Supercomputing 61(1), 189-214 (2012). doi:10.1007/s11227-011-0686-1

18 116. Mobius, C., Dargie, W., Schill, A.: Power Consumption Estimation Models for Processors, Virtual Machines, and

19 Servers. IEEE Transactions on Parallel and Distributed Systems 25(6), 1600-1614 (2014). 19 doi:10.1109/TPDS.2013.183

20 117. Environmental management — Life cycle assessment — Principles and framework. Standards ISO 14040:2006 (2006). https://www .iso.org/cms/render/live/en/sites/isoorg/contents/data/standard/03/74/37456.html

23 118. L.1310 : Energy efficiency metrics and measurement methods for telecommunication equipment (2017). Accessed 2020-07-27

24119. Schien, D., Shabajee, P., Yearworth, M., Preist, C.: Modeling and Assessing Variability in Energy Consumption 24 During the Use Stage of Online Multimedia Services. Journal of Industrial Ecology 17(6), 800-813 (2013). doi:10.1111/jiec.12065. tex.copyright: (c) 2013 by Yale University. Accessed 2020-07-27

26120. Measurement method for energy efficiency of network functions virtualization. Technical report (November 2018). 26 https://www .itu.int/rec/T-REC-L. 1361/en Accessed 2020-07-23

27 121. Hennessy, J.L., Patterson, D.A.: Computer Architecture: A Quantitative Approach. Morgan Kaufmann, ??? (2017)

28 122. Inoue, T., Aikebaier, A., Enokido, T., Takizawa, M.: Algorithms for Selecting Energy-Efficient Storage Servers in 28 Storage and Computation Oriented Applications. In: 2012 IEEE 26th International Conference on Advanced

29 Information Networking And Applications, pp. 920-927 (2012). doi:10.1109/AINA.2012.136

30 123. Al-Zubaedi, W., Al-Raweshidy, H.S.: A parameterized and optimized BBU pool virtualization power model for C-RAN architecture. In: IEEE EUROCON 2017 -17th International Conference on Smart Technologies, pp. 38-43 (2017).

31 doi:10.1109/EUROCON.2017.8011074

124. Mijumbi, R., Serrat, J., Gorricho, J.-L., Rubio-Loyola, J.: On the Energy Efficiency Prospects of Network Function Virtualization. arXiv:1512.00215 [cs] (2015). Accessed 2020-07-15 
2126. Dumitrescu, C.: Design Patterns for Packet Procesing Applications on Multi-core Intel Architecture (2008). Accessed 2 2020-09-29

3127. Hähnel, M., Döbel, B., Völp, M., Härtig, H.: Measuring energy consumption for short code paths using RAPL. ACM 3

4 SIGMETRICS Performance Evaluation Review 40(3), 13-17 (2012). doi:10.1145/2425248.2425252. Accessed 2020-07-27

5128. TelecomTV: The 5G Core is Vital to Deliver the Promise of 5G. TelecomTV (2020). Accessed 2021-02-17 5

129. Bhyrraju, R., Chu, H., Karlsson-Taylor, J.F., Druta, D., Brenner, M., Gautam, D., Kitroser, I., Wocalewski, T., Wright, S.: gs_NFV-IFA011v020701p.pdf, 2.7.1 edn. Network Functions Virtualisation (NFV) Release 2, vol. ETSI GS

$7 \quad$ NFV-IFA 011. ETSI, Sophia Antipolis Cedex - FRANCE (2019). https://www.etsi.org/deliver/etsi_gs/NFV-IFA/001_099/011/02.07.01_60/gs_NFV-IFA011v020701p.pdf Accessed 2021-02-18

9130. The NANOG February 2021 Archive by thread. NANOG Mailing List Archive (2021). Type: Mailing list archive. Accessed 2021-03-05

10131. Dargie, W.: A Stochastic Model for Estimating the Power Consumption of a Processor. IEEE Transactions on 10 Computers 64(5), 1311-1322 (2015). doi:10.1109/TC.2014.2315629

132. Garraghan, P., Moreno, I.S., Townend, P., Xu, J.: An Analysis of Failure-Related Energy Waste in a Large-Scale

12 Cloud Environment. IEEE Transactions on Emerging Topics in Computing 2(2), 166-180 (2014). 12 doi:10.1109/TETC.2014.2304500

13 133. Monteiro, A.F., Loques, O.: Quantum Virtual Machine: A Scalable Model to Optimize Energy Savings and Resource

14 Management. In: 2015 27th International Symposium on Computer Architecture and High Performance Computing 14 (SBAC-PAD), pp. 194-201 (2015). doi:10.1109/SBAC-PAD.2015.24

15 134. Sharma, N.K., Sharma, P., Guddeti, R.M.R.: Energy efficient quality of service aware virtual machine migration in 15

16 cloud computing. In: 2018 4th International Conference on Recent Advances in Information Technology (RAIT), pp. 16 1-6 (2018). doi:10.1109/RAIT.2018.8389047

17135. Zakarya, M., Gillam, L.: An Energy Aware Cost Recovery Approach for Virtual Machine Migration. In: Bañares, J.A., 17 Tserpes, K., Altmann, J. (eds.) Economics of Grids, Clouds, Systems, and Services. Lecture Notes in Computer Science, pp. 175-190. Springer, Cham (2017). doi:10.1007/978-3-319-61920-0_13

19136. Zhao, T., Wu, J., Zhou, S., Niu, Z.: Energy-delay tradeoffs of virtual base stations with a computational-resource-aware energy consumption model. In: 2014 IEEE International Conference on

138. Son, J., He, T., Buyya, R.: CloudSimSDN-NFV: Modeling and simulation of network function virtualization and service

22 function chaining in edge computing environments. Software: Practice and Experience 49(12), 1748-1764 (2019). 22 doi:10.1002/spe.2755. Accessed 2020-07-07

139. Slomon, D.A., Yosifovich, P., Russinovich, M.E., Lonescu, A.: System Architecture, Processes, Threads, Memory

24 Management, and More, 7th edn. Windows internals ; Part 1. Pearson Education, ??? (2017).

26141. Goraczko, M.: Joulemeter: Computational Energy Measurement and Optimization. Microsoft Research (2010). Type: 26 Blog. Accessed 2020-07-27

27 142. Lin, W., Wu, W., Wang, H., Wang, J.Z., Hsu, C.-H.: Experimental and quantitative analysis of server power model for 27

28 cloud data centers. Future Generation Computer Systems 86, 940-950 (2018). doi:10.1016/j.future.2016.11.034. 28 Accessed 2019-12-17

29143. Intel Core ${ }^{\mathrm{TM}}$ i5-3230M Processor (3M Cache, up to $3.20 \mathrm{GHz}$ ) rPGA Product Specifications (2014). Accessed 29 2020-07-27

30

144. Mobile 3rd Generation IntelCoreTM Processor Family, Mobile Intel Pentium Processor Family and Mobile Intel

31 Celeron Processor Family. Datasheet 326768-006 (June 2013). https://www . intel . com/content/dam/www/ public/us/en/documents/datasheets/3rd-gen-core-family-mobile-vol-1-datasheet.pdf Accessed 2020-07-27 
$2^{146 .}$ Chios, M., Clarke, D., Willis, P., Reid, A., Feger, J., Bugenhagen, M., Khan, W., Fargano, M., Cui, D.C., Deng, D.H., 2 Benitez, J., Michel, U., Damker, H., Ogak, K., Matsuzaki, T., Fukui, M., Shimano, K., Delisle, D., Loudier, Q., Kolias,

3 C., Guardini, I., Demaria, E., Minerva, R., Manzalini, A., López, D., Salguero, F.J.R., Ruhl, F., Sen, P.: Network 3

$4 \quad$ Functions Virtualisation. An Introduction, Benefits, Enablers, Challenges \& Call for Action. In: SDN and OpenFlow 4 World Congress, Darmstadt, Germany (2012)

5147. Molka, D., Hackenberg, D., Schöne, R., Müller, M.S.: Characterizing the energy consumption of data transfers and 5 arithmetic operations on x86-64 processors. In: International Conference on Green Computing, pp. 123-133 (2010). doi:10.1109/GREENCOMP.2010.5598316

7148. Rahman, A., Lynch, P., Aranda, P., Bernardos, C., Zuniga, J., Contreras, L.: Network Virtualization Research 7 Challenges. Request For Comments (RFC) - Informational RFC 8568, ISSN: 2070-1721 (April 2019). https://tools.ietf.org/html/rfc8568\#page-18 Accessed 2020-07-10

9149. Dahyun: SR-IOV for NFV solutions - practical considerations and thoughts (2017). Accessed 2020-07-27

150. Shuja, J., Bilal, K., Madani, S.A., Othman, M., Ranjan, R., Balaji, P., Khan, S.U.: Survey of Techniques and Architectures for Designing Energy-Efficient Data Centers. IEEE Systems Journal 10(2), 507-519 (2016). doi:10.1109/JSYST.2014.2315823

151. Mastelic, T., Oleksiak, A., Claussen, H., Brandic, I., Pierson, J.-M., Vasilakos, A.V.: Cloud Computing: Survey on

12 Energy Efficiency. ACM Computing Surveys 47(2), 33-13336 (2014). doi:10.1145/2656204. Accessed 2021-03-02 12

152. Ismail, M., Zhuang, W., Serpedin, E., Qaraqe, K.: A Survey on Green Mobile Networking: From The Perspectives of Network Operators and Mobile Users. IEEE Communications Surveys Tutorials 17(3), 1535-1556 (2015).

153. Bianzino, A.P., Chaudet, C., Rossi, D., Rougier, J.: A Survey of Green Networking Research. IEEE Communications

16 154. Faraci, G., Schembra, G.: An Analytical Model to Design and Manage a Green SDN/NFV CPE Node. IEEE Transactions on Network and Service Management 12(3), 435-450 (2015). doi:10.1109/TNSM.2015.2454293

17155. Tuysuz, M.F., Ankarali, Z.K., Gözüpek, D.: A survey on energy efficiency in software defined networks. Computer 17 Networks 113, 188-204 (2017). doi:10.1016/j.comnet.2016.12.012. Accessed 2021-03-02

156. Qi, D., Shen, S., Wang, G.: Virtualized Network Function Consolidation Based on Multiple Status Characteristics.

19 IEEE Access 7, 59665-59679 (2019). doi:10.1109/ACCESS.2019.2915919

157. Kim, S., Park, S., Kim, Y., Kim, S., Lee, K.: VNF-EQ: dynamic placement of virtual network functions for energy

20 efficiency and QoS guarantee in NFV. Cluster Computing 20(3), 2107-2117 (2017). doi:10.1007/s10586-017-1004-3. Accessed 2021-03-02

158. Bolla, R., Bruschi, R., Davoli, F., Lombardo, C.: Fine-Grained Energy-Efficient Consolidation in SDN Networks and Devices. IEEE Transactions on Network and Service Management 12(2), 132-145 (2015). doi:10.1109/TNSM.2015.2431074

159. Akyildiz, I.F., Kak, A., Nie, S.: 6 G and Beyond: The Future of Wireless Communications Systems. IEEE Access 8 ,

24 133995-134030 (2020). doi:10.1109/ACCESS.2020.3010896

. Smart Networks in the Context of NGI. Technical report, Expert Advisory Group of the European Technology Platform Networld 2020 (September 2020).

26 https://bscw.5g-ppp.eu/pub/bscw.cgi/d367342/Networld2020SRIAFinalVersion2.2.pdf Accessed 2021-03-18

27 161. Rossi, F.D., Xavier, M.G., De Rose, C.A.F., Calheiros, R.N., Buyya, R.: E-eco: Performance-aware energy-efficient 27 cloud data center orchestration. Journal of Network and Computer Applications 78, 83-96 (2017). doi:10.1016/j.jnca.2016.10.024. Accessed 2020-07-27

29162. Chongya Ma, Zhiying Jiang, Ke Zhang, Guangfei Zhang, Zhixiong Jiang, Chunyang Lu, Yushan Cai: Virtual machine 29 power metering and its applications. In: 2013 IEEE Global High Tech Congress on Electronics, pp. 153-156. IEEE Shenzhen (2013). doi:10.1109/GHTCE.2013.6767262.

31 http://ieeexplore.ieee.org//pdocs/epic03/wrapper.htm?arnumber=6767262 Accessed 2018-09-09

Wu, X., Zeng, Y., Lin, G.: An Energy Efficient VM Migration Algorithm in Data Centers. In: 2017 16th International Symposium on Distributed Computing and Applications to Business, Engineering and Science (DCABES), pp. 
2164. Carrega, A., Portomauro, G., Repetto, M., Robino, G.: Boosting Energy Efficiency and Quality of Service through 2 Orchestration Tools. IEEE Cloud Computing 5(6), 38-47 (2018). doi:10.1109/MCC.2018.064181119

3165. Rossi, F., Xavier, M., De Rose, C., Calheiros, R., Buyya, R.: E-eco: Performance-Aware Energy-Efficient Cloud Data 3 Center Orchestration. Journal of Network and Computer Applications 78 (2016). doi:10.1016/j.jnca.2016.10.024

166. Wen, C., Mu, Y.: Power and Performance Management in Nonlinear Virtualized Computing Systems via Predictive

$5 \quad$ Control. PLOS ONE 10(7), 0134017 (2015). doi:10.1371/journal.pone.0134017 5

167. Swarm mode overview. Docker Documentation (2019). Accessed 2019-10-08

6 168. Production-Grade Container Orchestration (2021). Accessed 2019-10-08

7169. Apache Mesos. Apache Mesos (2021). Accessed 2019-10-08

170. Devi, D.C., Uthariaraj, V.R.: Load Balancing in Cloud Computing Environment Using Improved Weighted Round Robin Algorithm for Nonpreemptive Dependent Tasks. The Scientific World Journal 2016, 1-14 (2016).

171. Hinz, M., Koslovski, G.P., Miers, C.C., Pilla, L.L., Pillon, M.A.: A Cost Model for laaS Clouds Based on Virtual

10 Machine Energy Consumption. Journal of Grid Computing 16(3), 493-512 (2018). doi:10.1007/s10723-018-9440-8. 10

11 Accessed 2019-10-08

172. Bolla, R., Bruschi, R., Davoli, F., Di Gregorio, L., Donadio, P., Fialho, L., Collier, M., Lombardo, A.,

12 Reforgiato Recupero, D., Szemethy, T.: The Green Abstraction Layer: A Standard Power-Management Interface for 12 Next-Generation Network Devices. IEEE Internet Computing 17(2), 82-86 (2013). doi:10.1109/MIC.2013.39.

14173. L.1362 : Interface for power management in network function virtualization environments - Green abstraction Layer 14 version 2 (2019). Accessed 2021-02-21

15 174. Network Functions Virtualisation (NFV); Management and Orchestration. Technical Report DGS/NFV-MAN001, ETSI, Sophia Antipolis Cedex - FRANCE (December 2014)

175. Laganà, D., Mastroianni, C., Meo, M., Renga, D.: Reducing the Operational Cost of Cloud Data Centers through

17 Renewable Energy. Algorithms 11(10), 145 (2018). doi:10.3390/a11100145. Accessed 2019-10-08 17

176. Forestiero, A., Mastroianni, C., Meo, M., Papuzzo, G., Sheikhalishahi, M.: Hierarchical Approach for Efficient Workload Management in Geo-Distributed Data Centers. IEEE Transactions on Green Communications and Networking 1(1), 97-111 (2017). doi:10.1109/TGCN.2016.2603586. Accessed 2019-10-08

177. Schmitt, N., von Kistowski, J., Kounev, S.: Towards a Scalability and Energy Efficiency Benchmark for VNF. In: Nambiar, R., Poess, M. (eds.) Performance Evaluation and Benchmarking for the Analytics Era. Lecture Notes in

21 Computer Science, pp. 41-54. Springer, Cham (2018). doi:10.1007/978-3-319-72401-0_4

178. Chen, G., Bai, X., Huang, X., Li, M., Zhou, L.: Evaluating services on the cloud using ontology QoS model. In:

22 Proceedings of 2011 IEEE 6th International Symposium on Service Oriented System (SOSE), pp. $312-317$ (2011). 22

179. Cloud computing performance report. Technical report (2010)

24180. Benchmark evaluation of 114 public clouds. Technical report (2014)

181. Hwang, K., Bai, X., Shi, Y., Li, M., Chen, W., Wu, Y.: Cloud Performance Modeling and Benchmark Evaluation of Elastic Scaling Strategies. IEEE Transactions on Parallel and Distributed Systems 27, 1-1 (2015).

182. Yu, S., Yang, H., Wang, R., Luan, Z., Qian, D.: Evaluating architecture impact on system energy efficiency. PLoS ONE 12(11) (2017). doi:10.1371/journal.pone.0188428. tex.pmcid: PMC5697812. Accessed 2020-07-27

28 183. Noureddine, A., Bourdon, A., Rouvoy, R., Seinturier, L.: A preliminary study of the impact of software engineering on 28 GreenIT. In: 2012 First International Workshop on Green and Sustainable Software (GREENS), pp. 21-27. IEEE,

29 Zurich, Switzerland (2012). doi:10.1109/GREENS.2012.6224251. http://ieeexplore.ieee.org/document/6224251/ 29 Accessed 2019-10-23

184. Jiang, C., Wang, Y., Ou, D., Li, Y., Zhang, J., Wan, J., Luo, B., Shi, W.: Energy efficiency comparison of hypervisors.

31 Sustainable Computing: Informatics and Systems 22, 311-321 (2019). doi:10.1016/j.suscom.2017.09.005. Accessed31 2020-07-27

32 185. Lewis, A., Ghosh, S., Tzeng, N.-F.: Run-time energy consumption estimation based on workload in server systems. 32

33 In: Proceedings of the 2008 Conference on Power Aware Computing and Systems. HotPower'08, p. 4. USENIX 
2186. Eramo, V., Ammar, M., Lavacca, F.G.: Migration Energy Aware Reconfigurations of Virtual Network Function 2 Instances in NFV Architectures. IEEE Access 5, 4927-4938 (2017). doi:10.1109/ACCESS.2017.2685437

3187. Gu, C., Huang, H., Jia, X.: Power Metering for Virtual Machine in Cloud Computing-Challenges and Opportunities. 3 IEEE Access 2, 1106-1116 (2014). doi:10.1109/ACCESS.2014.2358992

188. OProfile - A System Profiler for Linux (News) (2020). Accessed 2020-07-27

5189. Sebastien, G.: sysstat/sysstat (2020). tex.copyright: GPL-2.0 License, GPL-2.0 License. Accessed 2020-07-27 5

190. Gregg, B.: Linux perf Examples (2018). Accessed 2019-08-13

91. Netto, A.Z Arnold, R.S. Evaluate performance for Linux on POWER (2012). tex.copyright: Copyright IBM

7 Corporation 2012. Accessed 2020-07-27

L., Rouvoy, R., Sobe, A.: Process-level power estimation in VM-based systems. In: Proceedings of the Tenth European Conference on Computer Systems - EuroSys '15, pp. 1-14. ACM 8 http://dl.acm.org/citation.cfm?doid=2741948.2741971 Accessed 2020-10-24

10193. G.W.A.T.T. (Global 'What if' Analyzer of NeTwork Energy ConsumpTion). Bell Labs application able to measure the 10 impact of technologies like SDN \& NFV on network energy consumption. (2015). https://media-bell-labs-com.s3.amazonaws.com/pages/20150114_1907/GWATT_WhitePaper.pdf

12194. Singh, A.K., Leech, C., Reddy, B.K., Al-Hashimi, B.M., Merrett, G.V.: Learning-Based Run-Time Power and Energy 12 Management of Multi/Many-Core Systems: Current and Future Trends. Journal of Low Power Electronics 13(3), 310-325 (2017). doi:10.1166/jolpe.2017.1492. Accessed 2019-10-08

14195. Ismail, L., Abed, E.H.: Linear Power Modeling for Cloud Data Centers: Taxonomy, Locally Corrected Linear 14 Regression, Simulation Framework and Evaluation. IEEE Access 7, 175003-175019 (2019).

15 doi:10.1109/ACCESS.2019.2956881

16 196. Tsai, C.-W., Lai, C.-F., Chao, H.-C., Vasilakos, A.: Big data analytics: A survey. Journal of Big Data 2 (2015). doi:10.1186/s40537-015-0030-3

17197. Stockman, M., Awad, M., Khanna, R., Le, C., David, H., Gorbatov, E., Hanebutte, U.: A novel approach to memory 17 power estimation using machine learning, pp. 1-3 (2011). doi:10.1109/ICEAC.2010.5702284

198. Braun, V., Clarke, V.: Using thematic analysis in psychology. Qualitative Research in Psychology 3(2), 77-101 Evaluation in R., p. 775. Rutgers Cooperative Extension, New Brunswick, NJ (2016). https://rcompanion.org/handbook/G 10.html

200. Sorzano, C.O.S., Vargas, J., Montano, A.P.: A survey of dimensionality reduction techniques. arXiv:1403.2877 [cs, q-bio, stat] (2014). Accessed 2020-07-27 


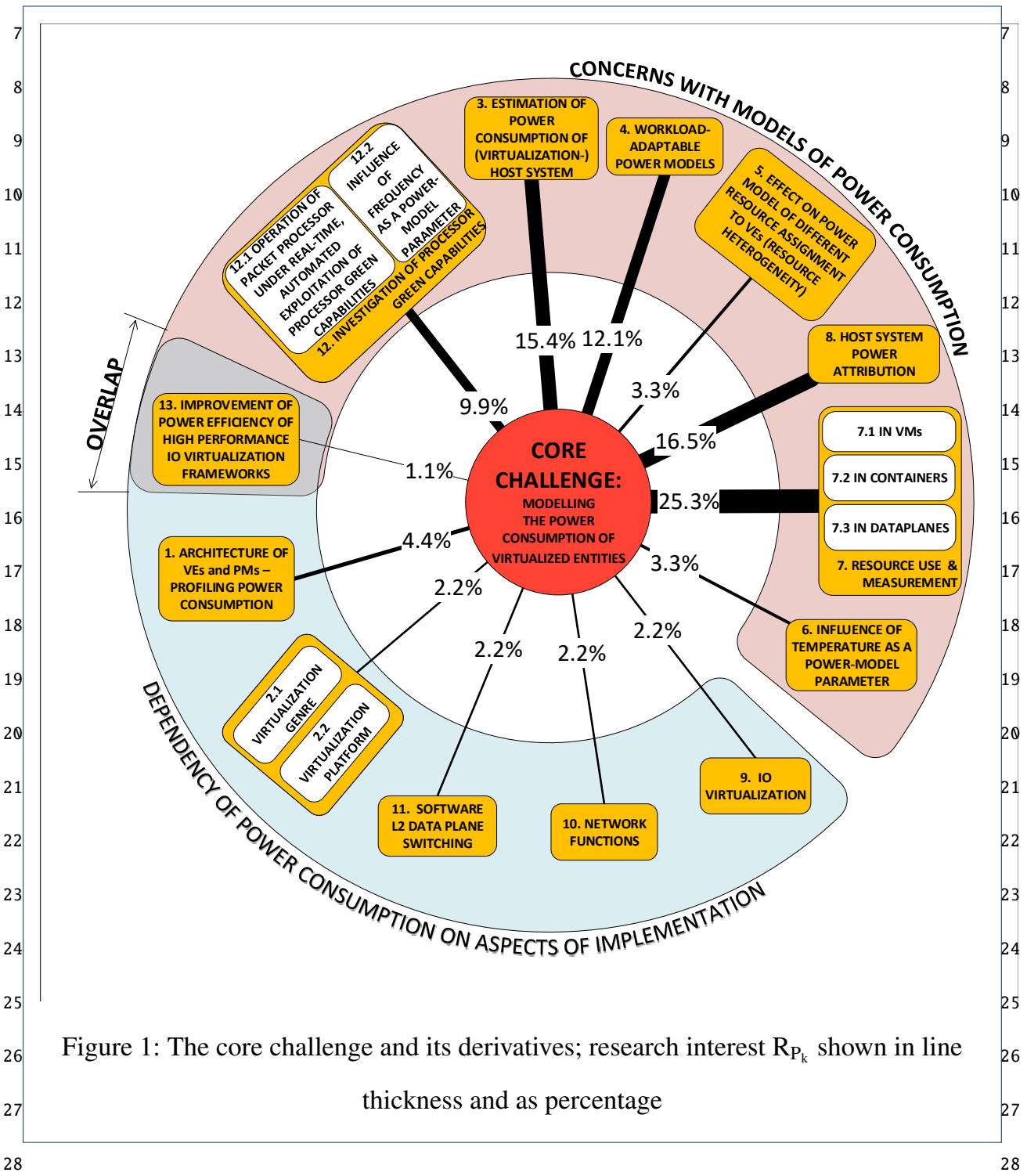




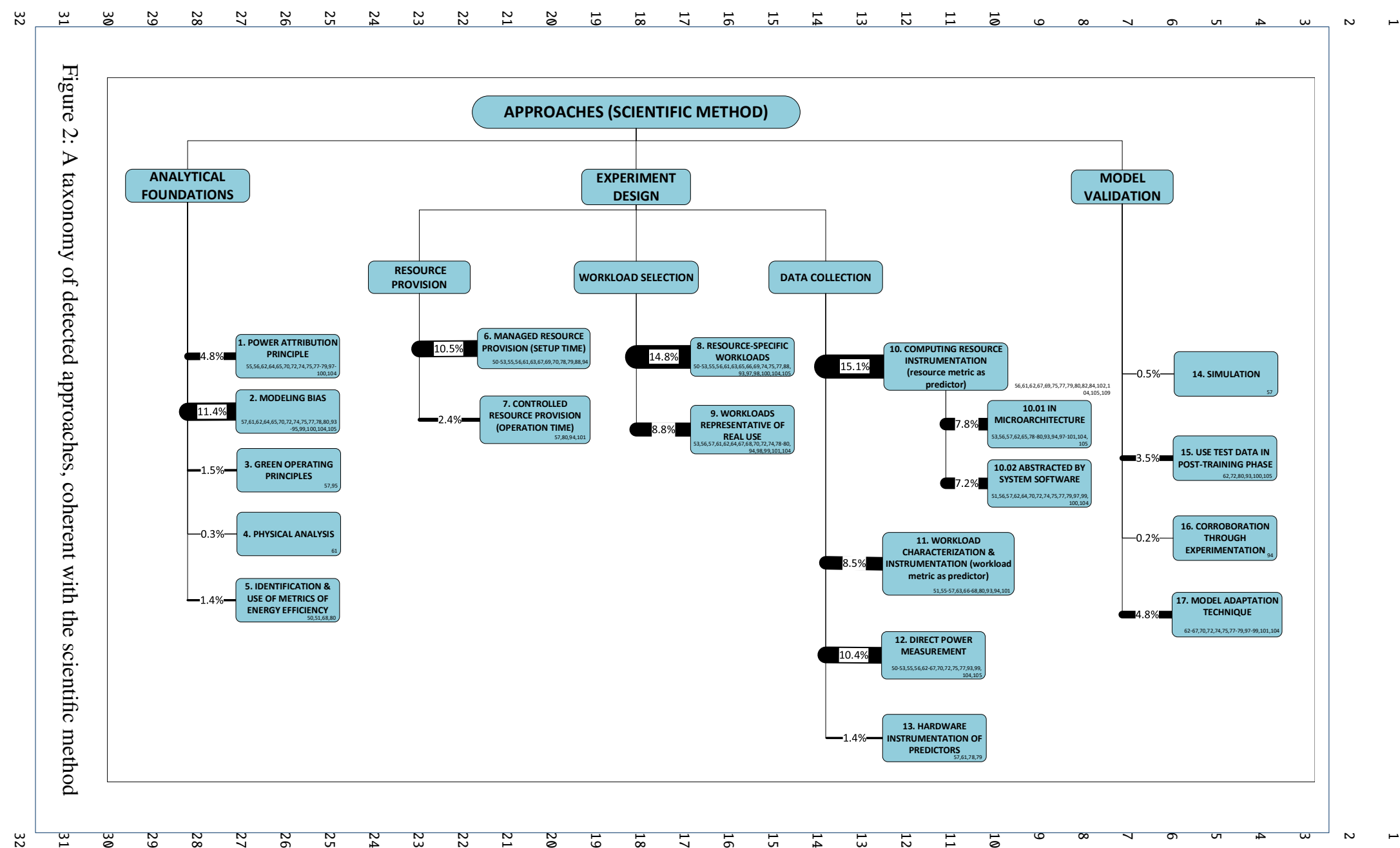



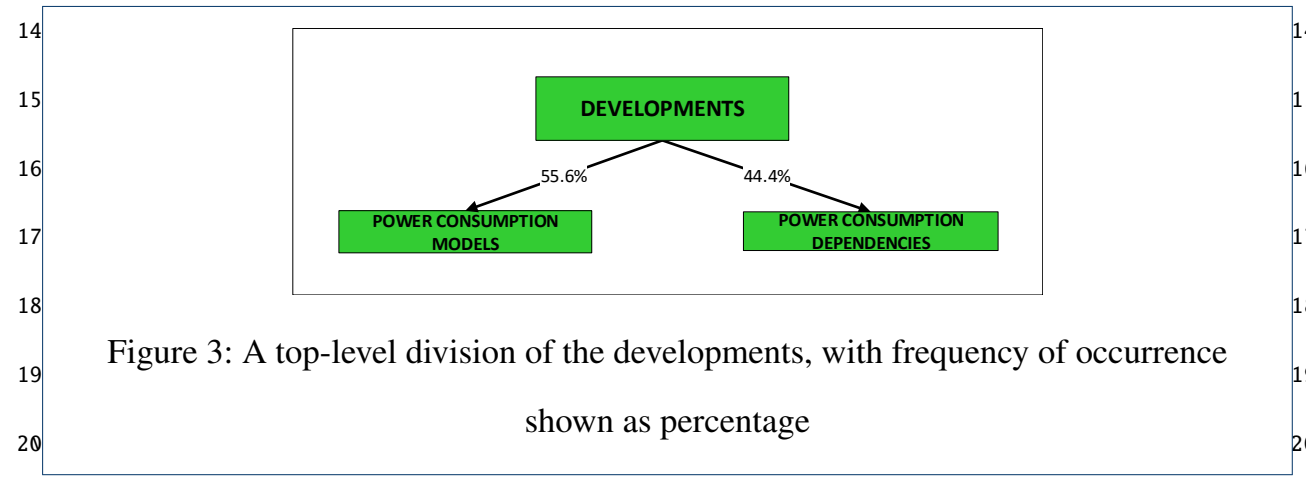

Figure 3: A top-level division of the developments, with frequency of occurrence shown as percentage 


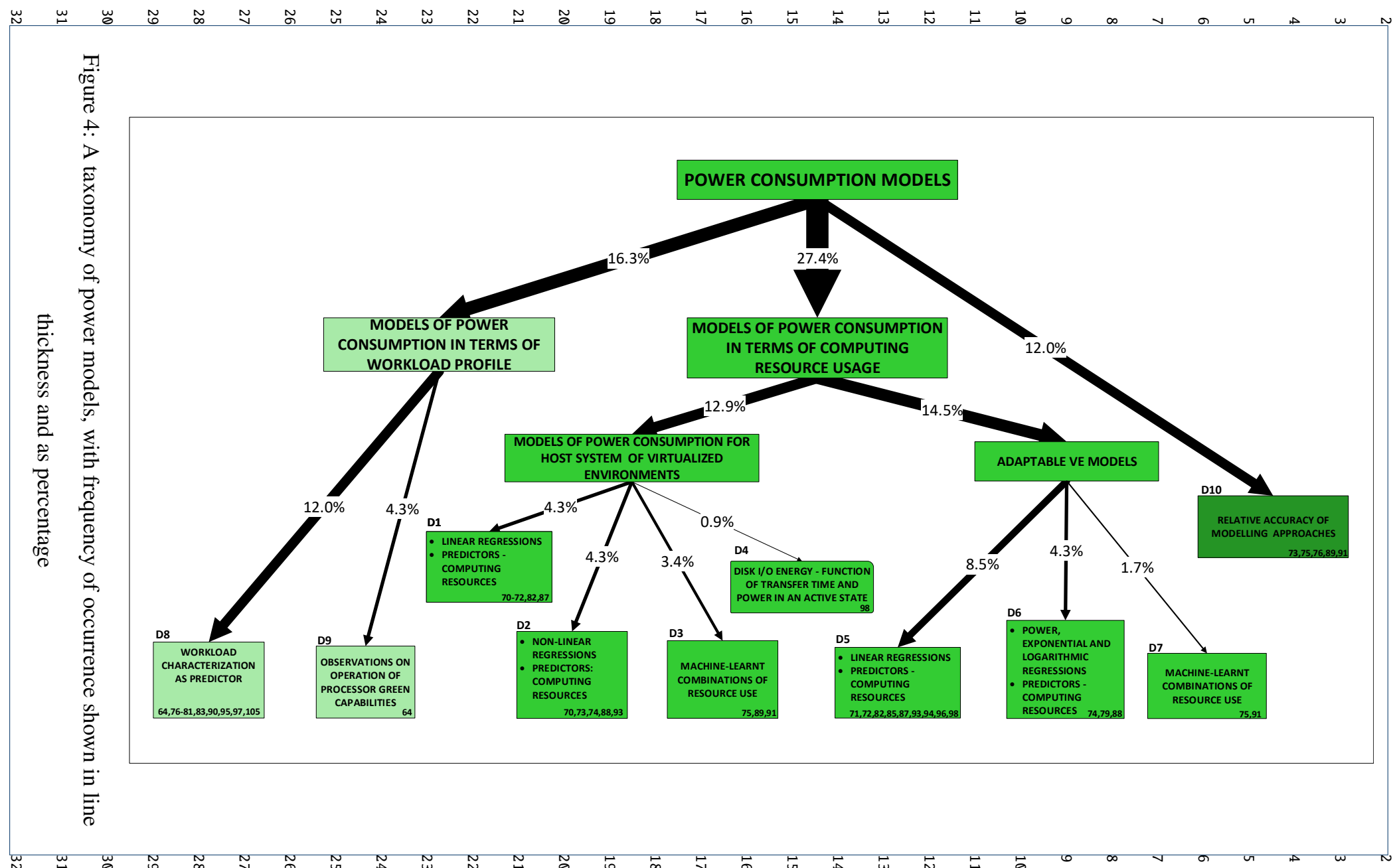



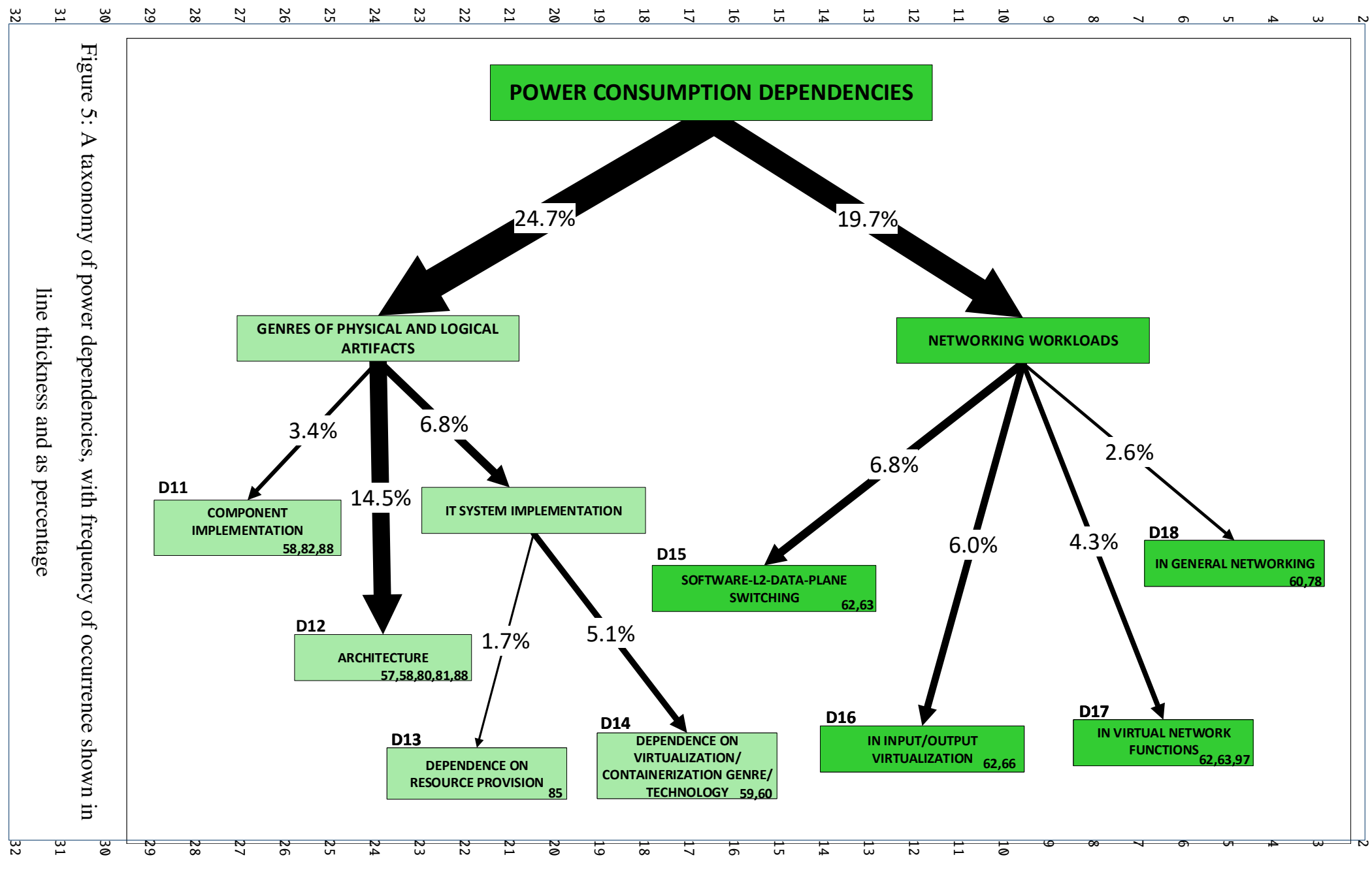


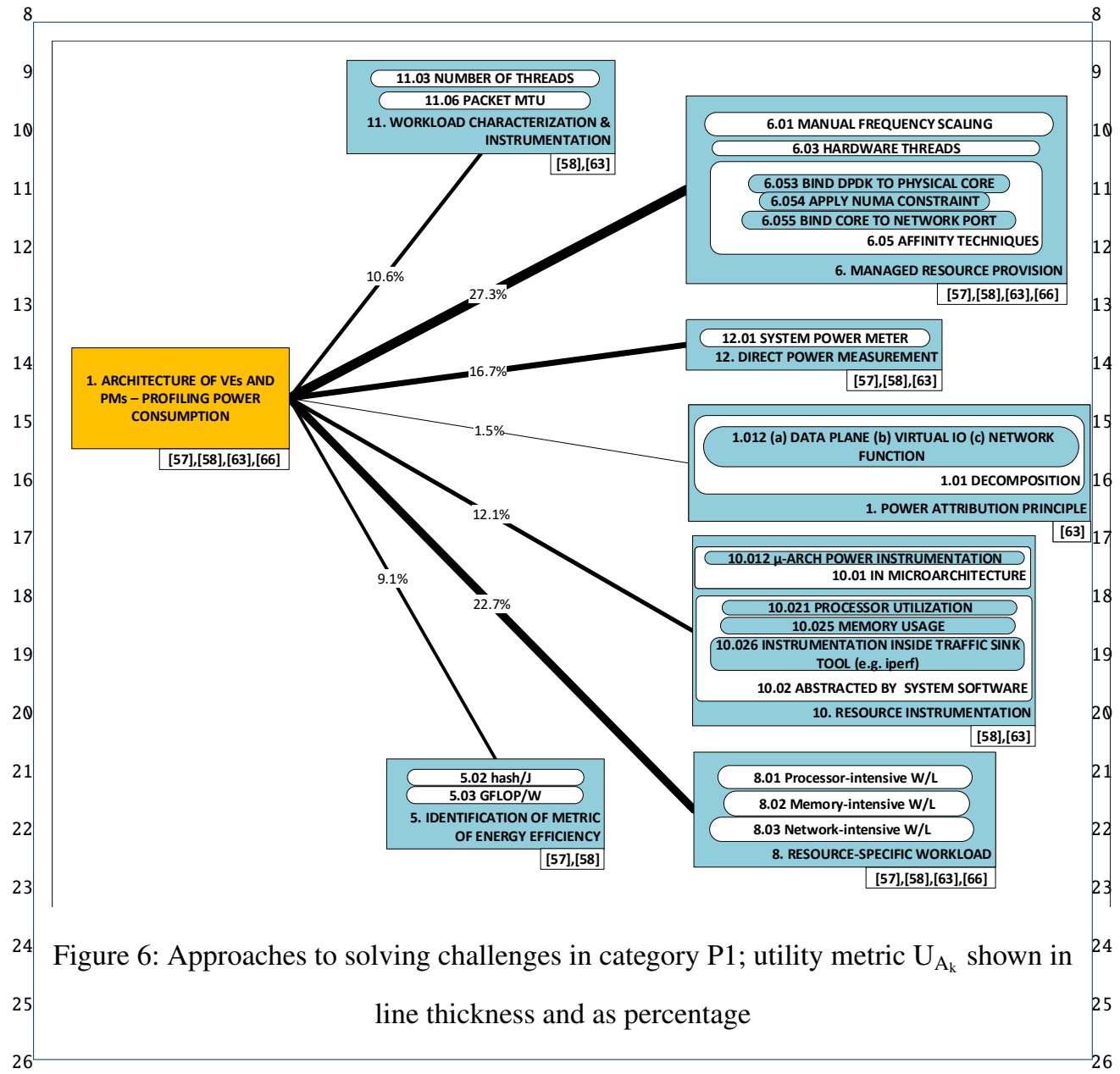




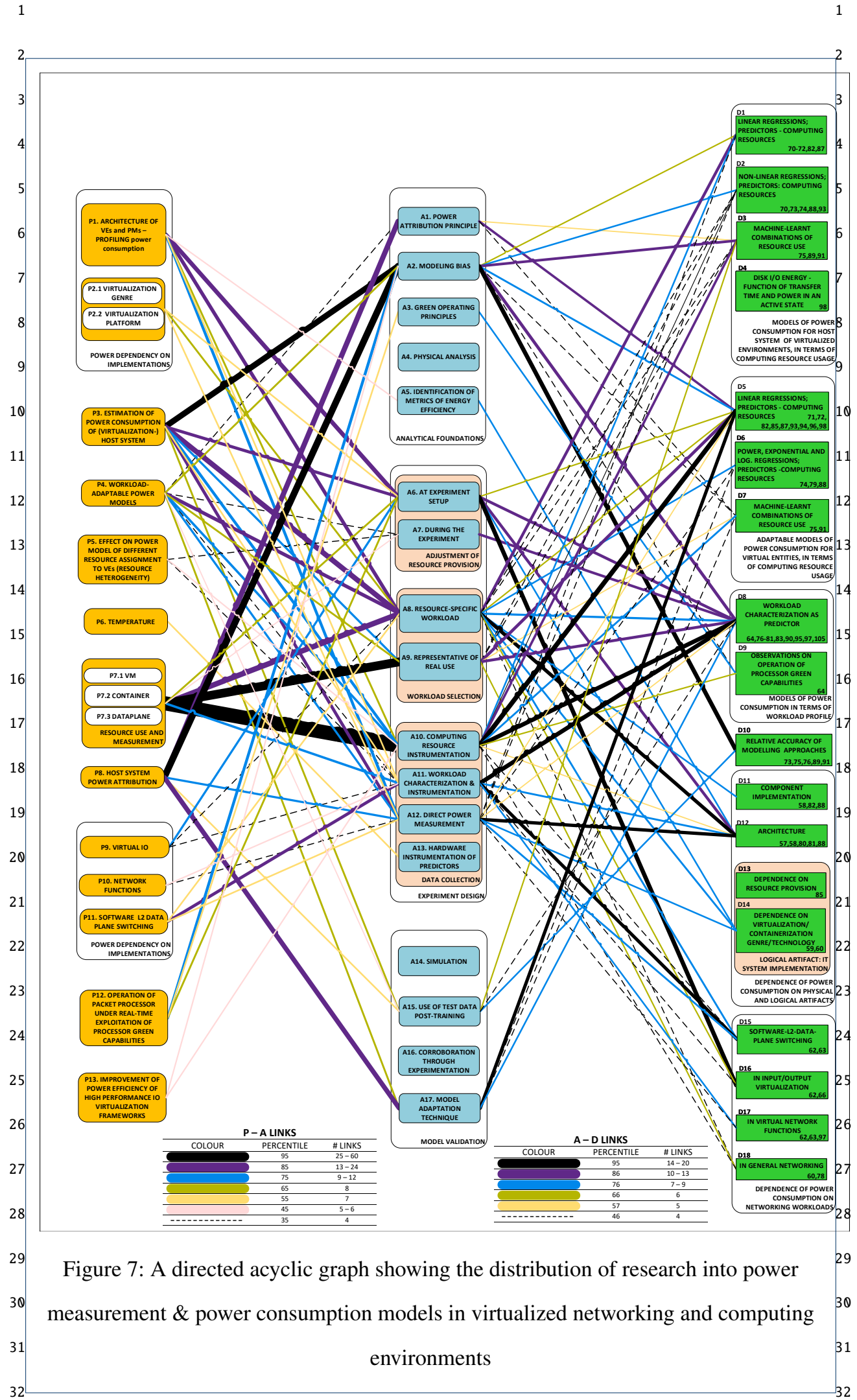



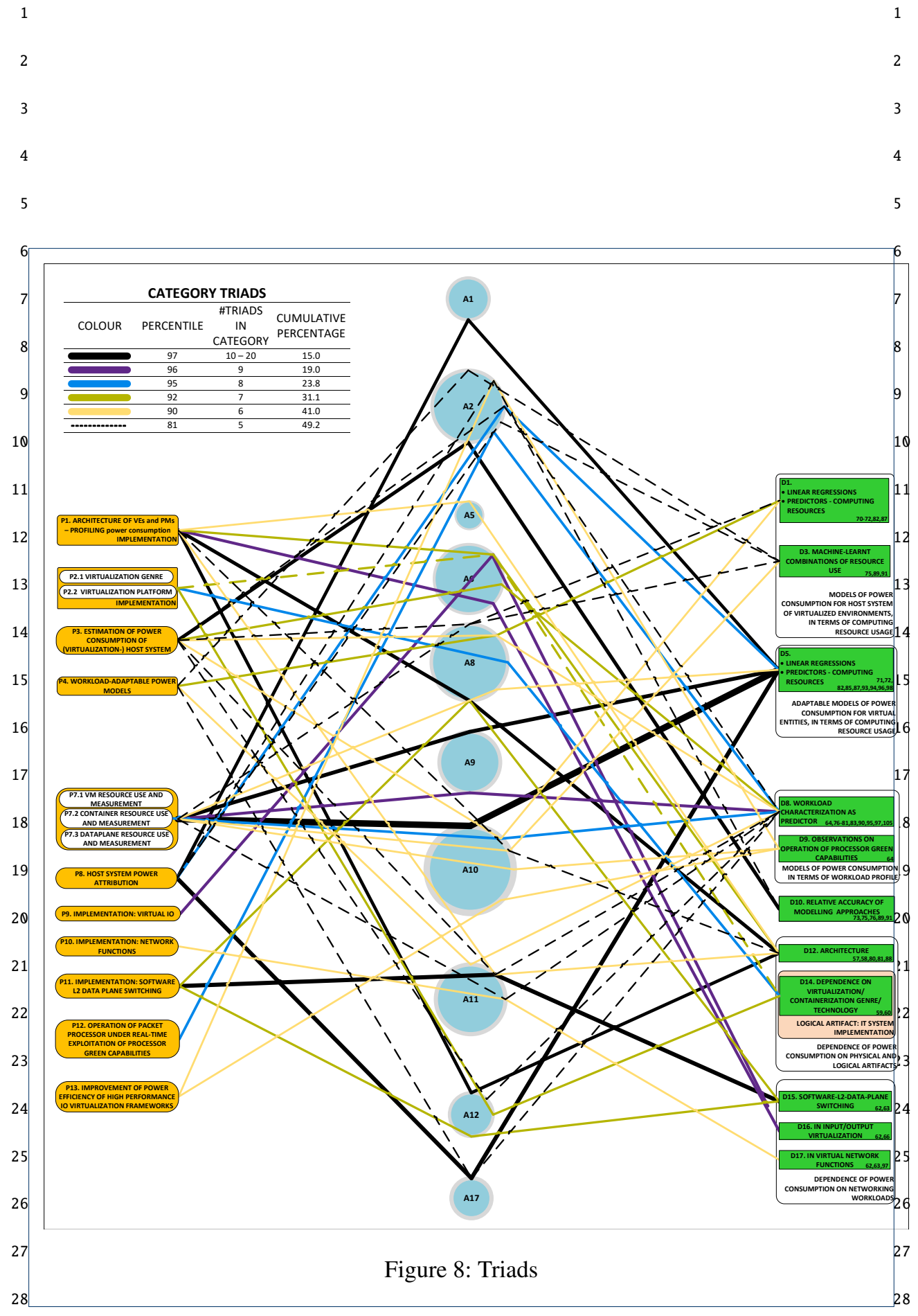

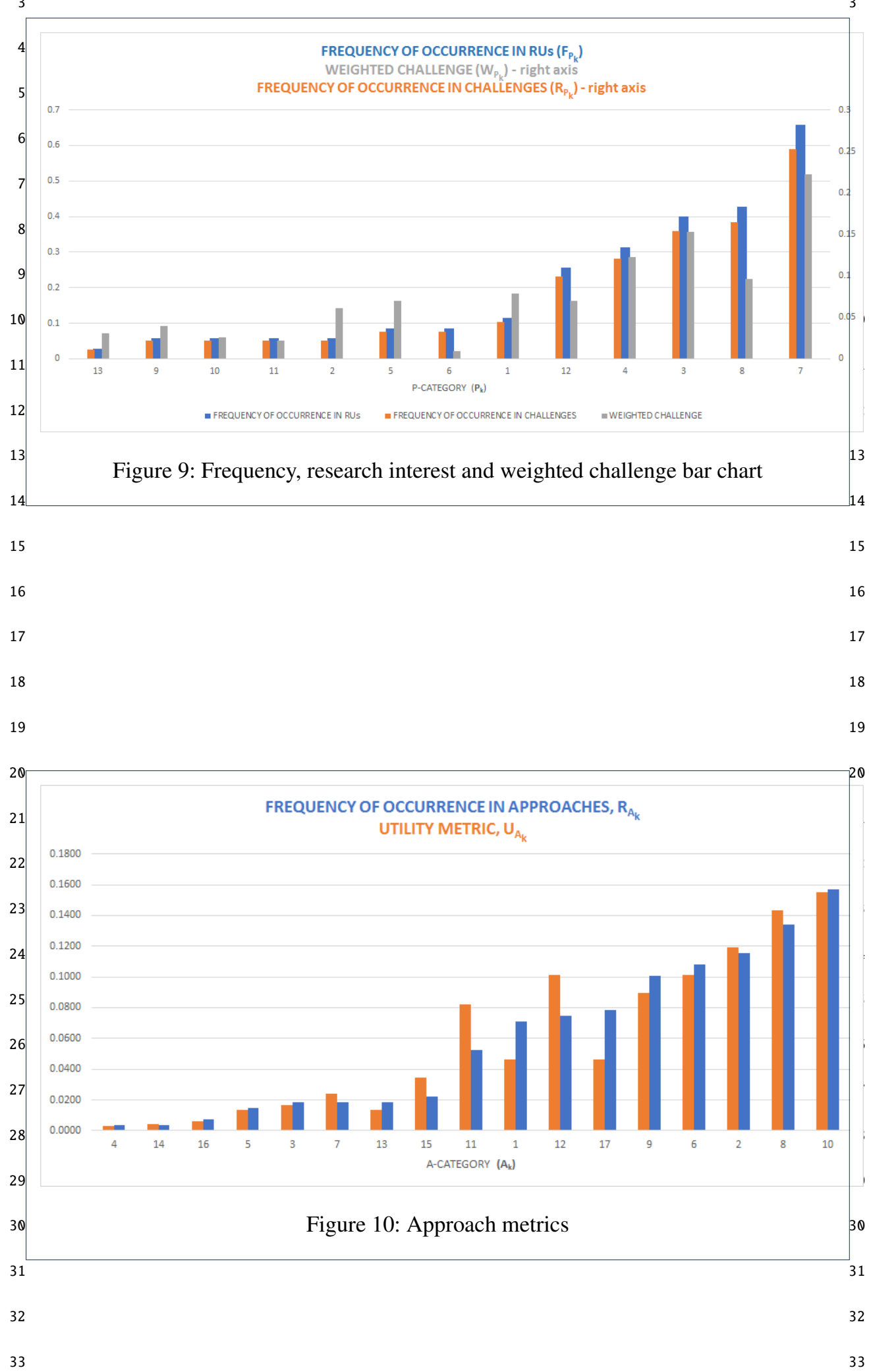

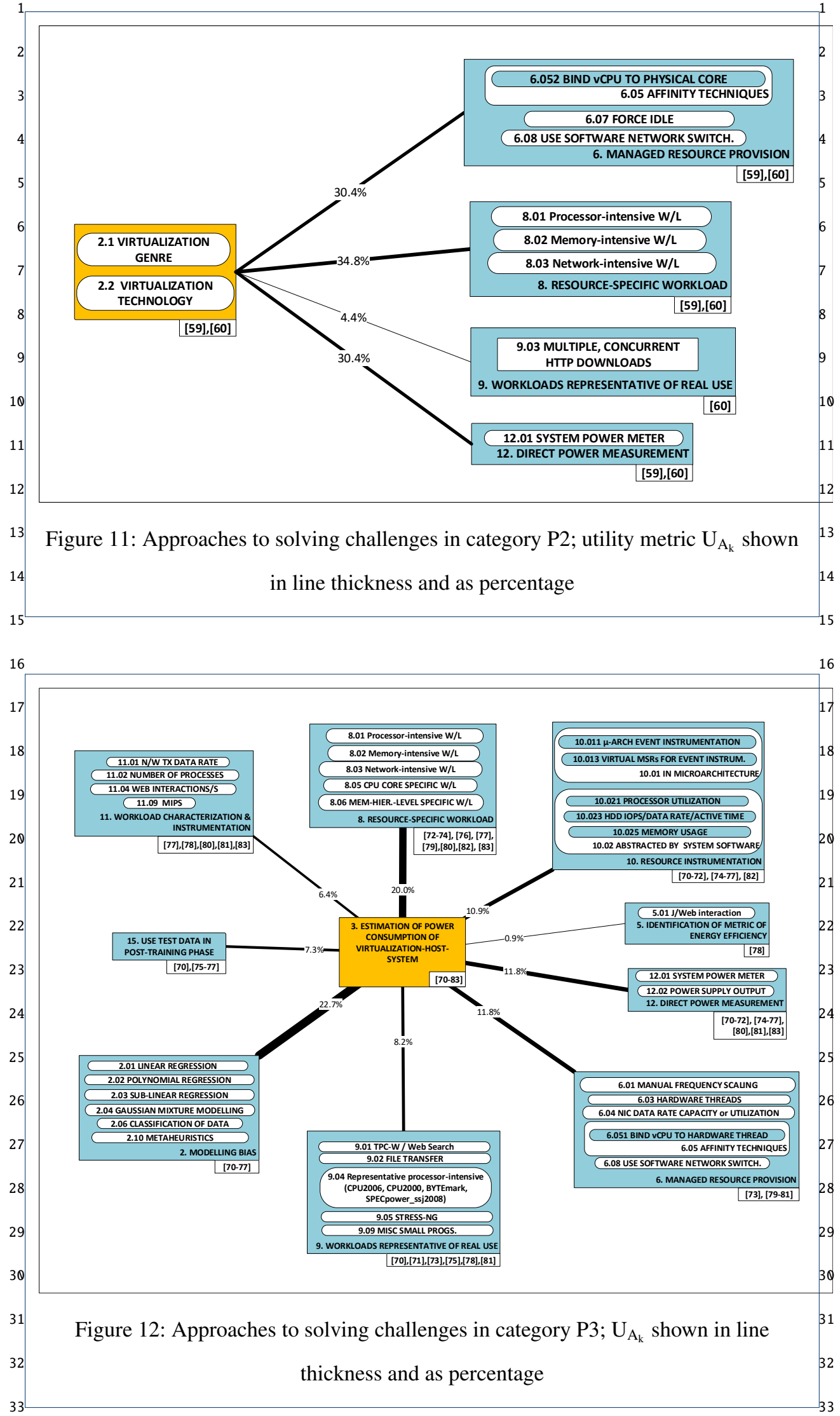

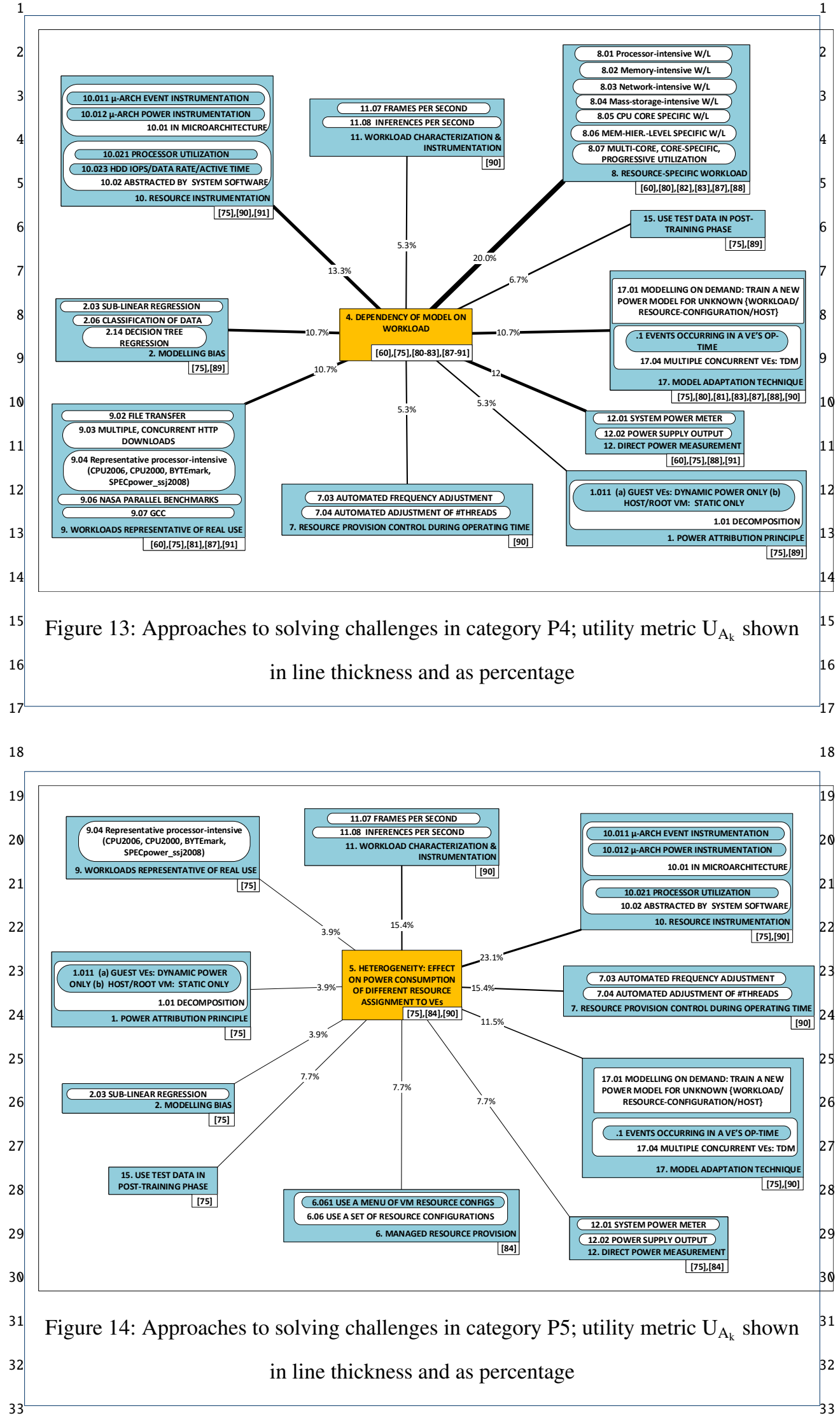


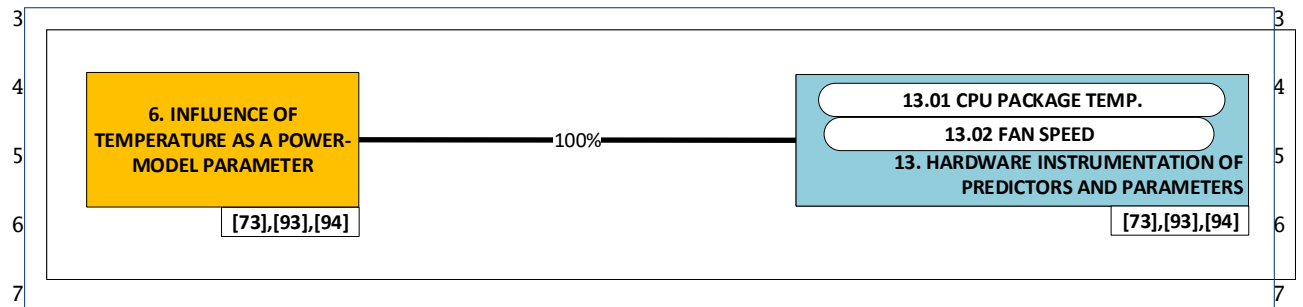

Figure 15: Approaches to solving challenges in category P6; utility metric $\mathrm{U}_{\mathrm{A}_{k}}$ shown in line thickness and as percentage

Figure 16: Approaches to solving challenges in category P7; utility metric $\mathrm{U}_{\mathrm{A}_{\mathrm{k}}}$ shown in line thickness and as percentage 


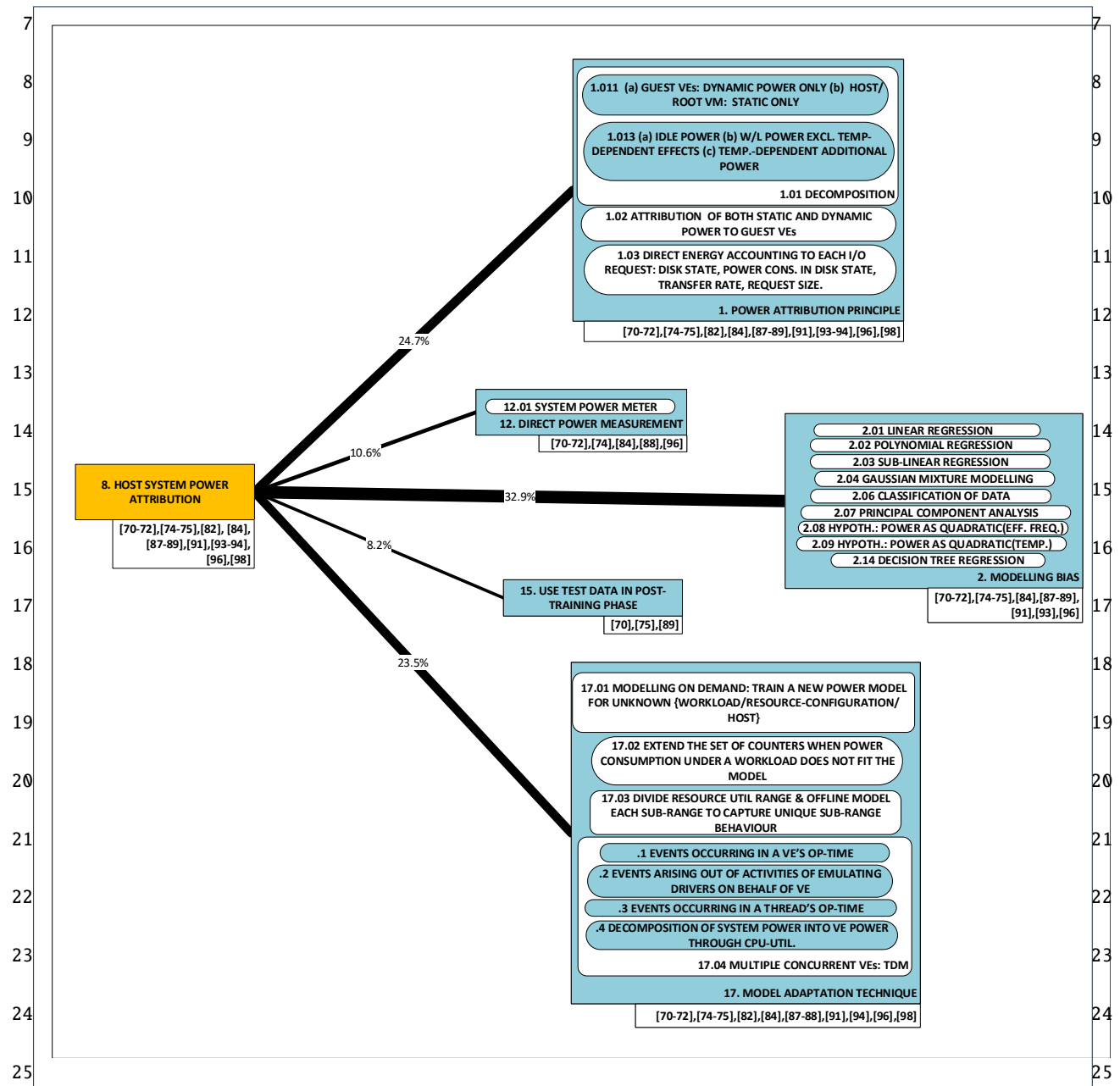

Figure 17: Approaches to solving challenges in category P8; utility metric $\mathrm{U}_{\mathrm{A}_{k}}$ shown in line thickness and as percentage 


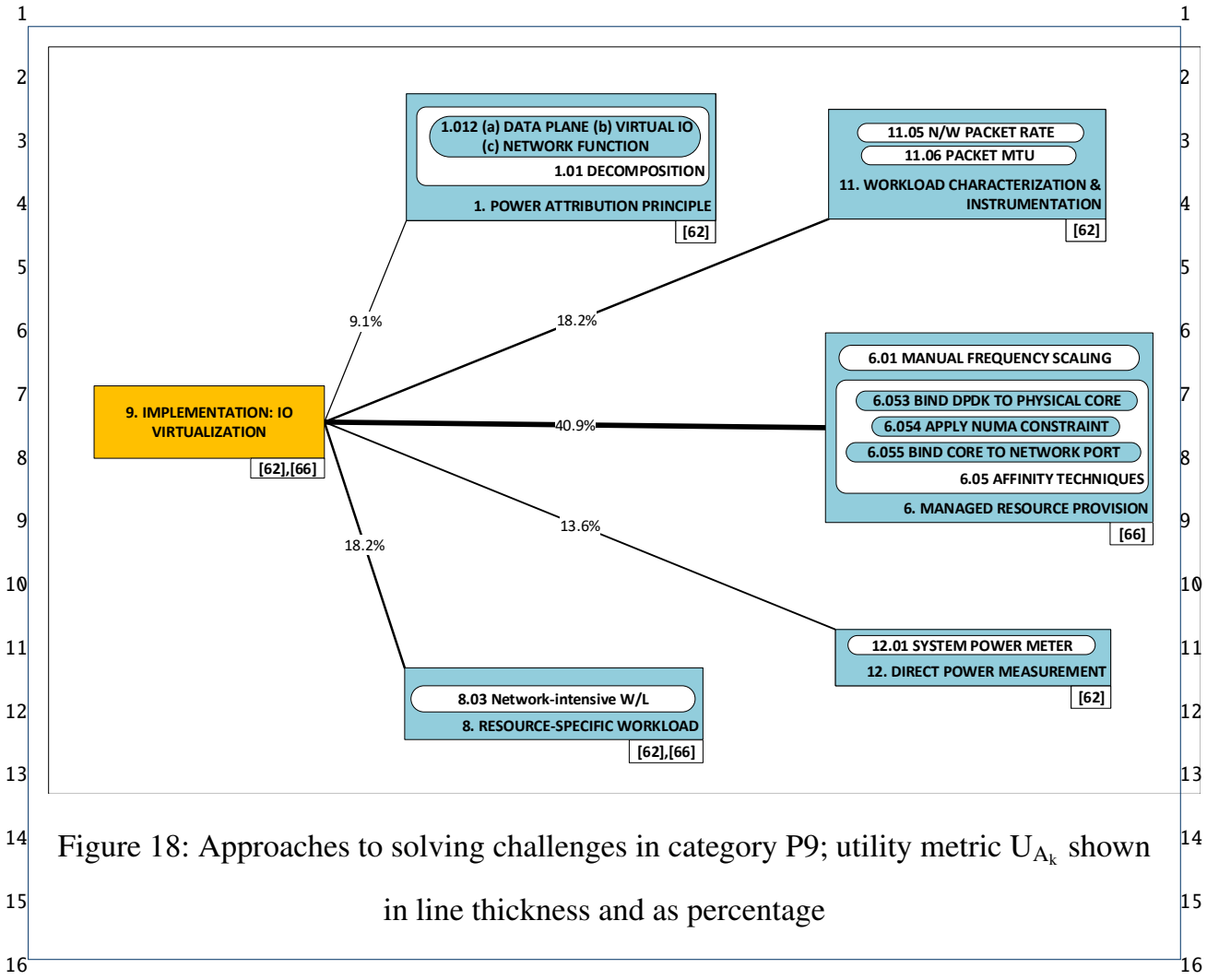

17

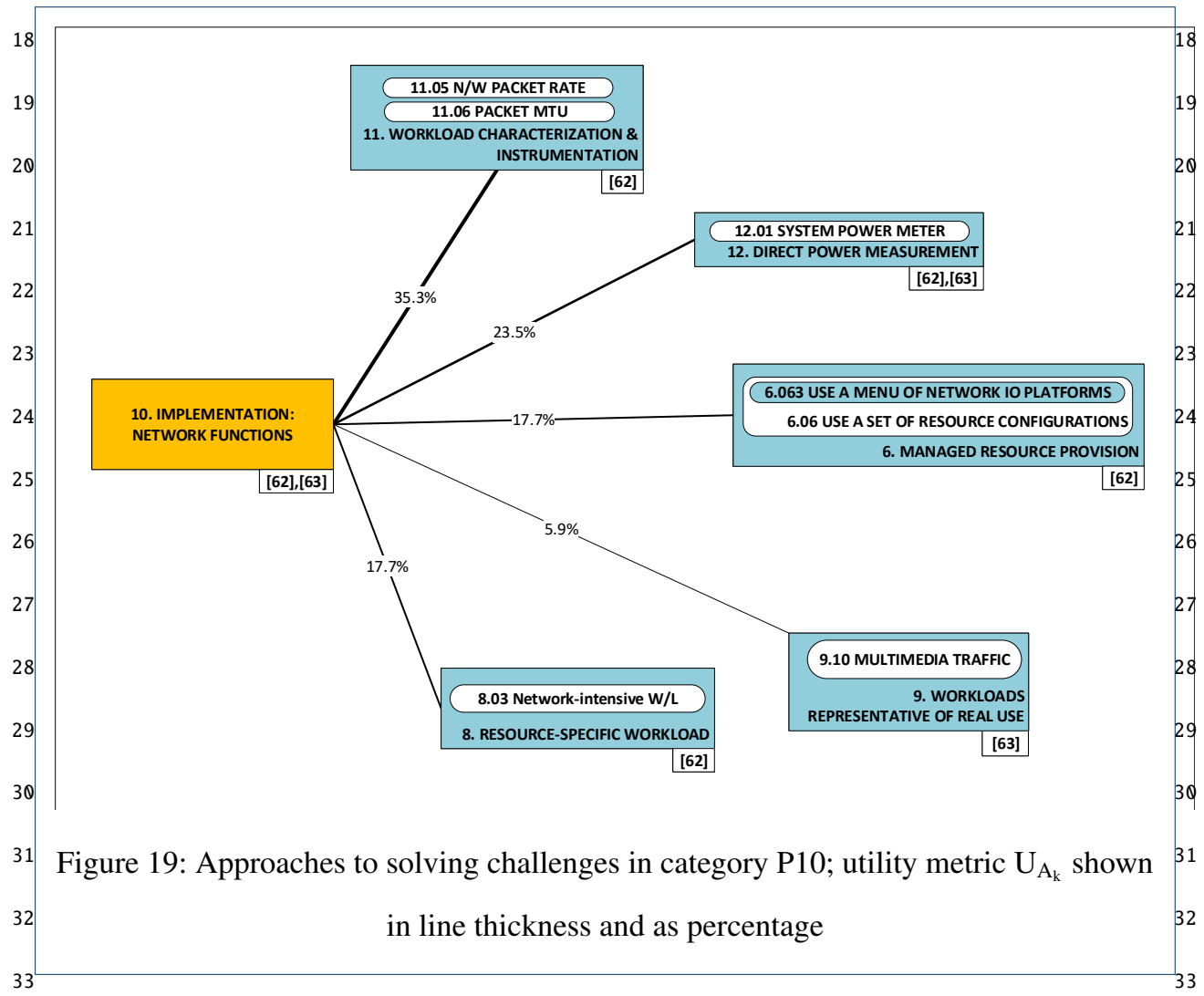




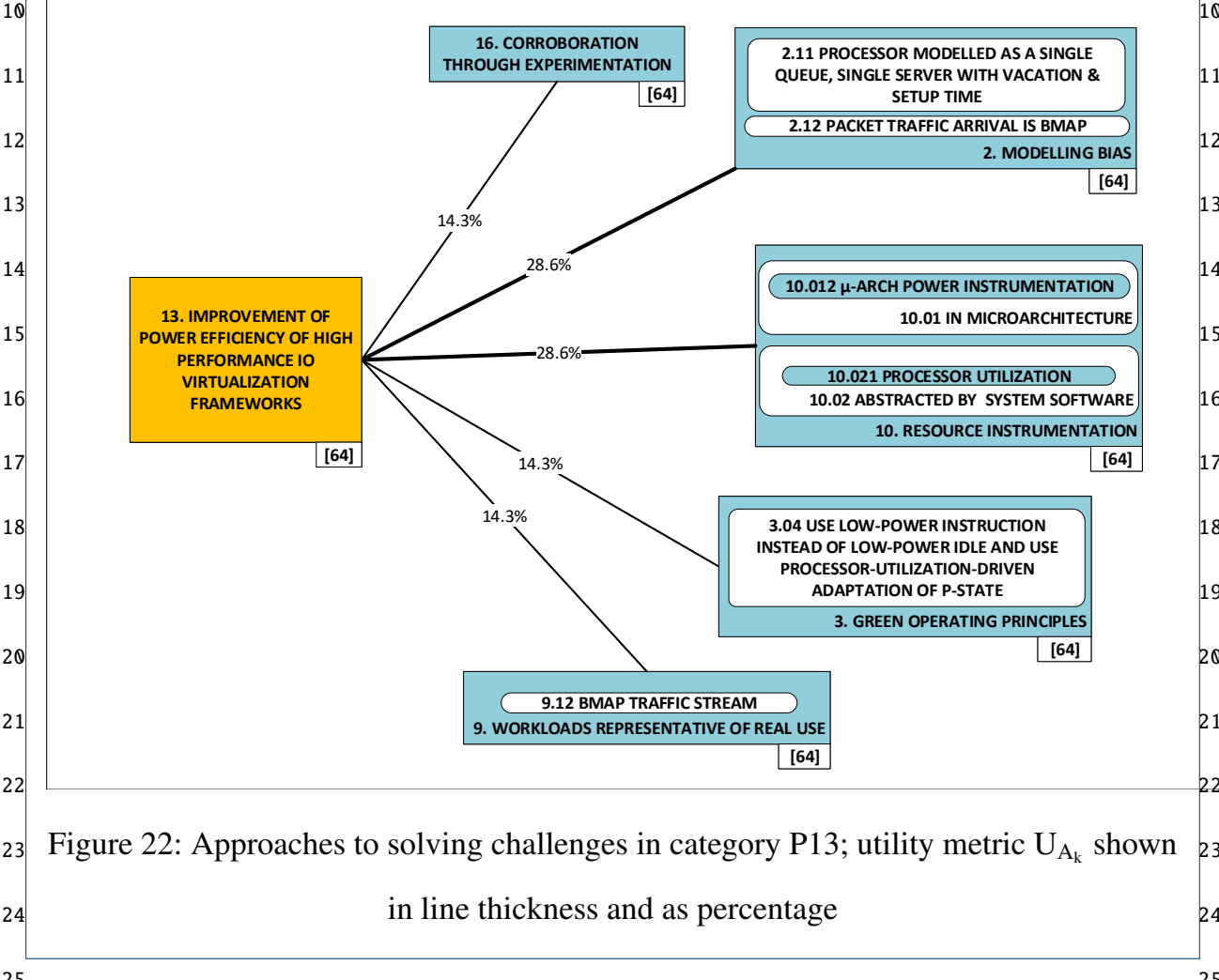




\section{9}

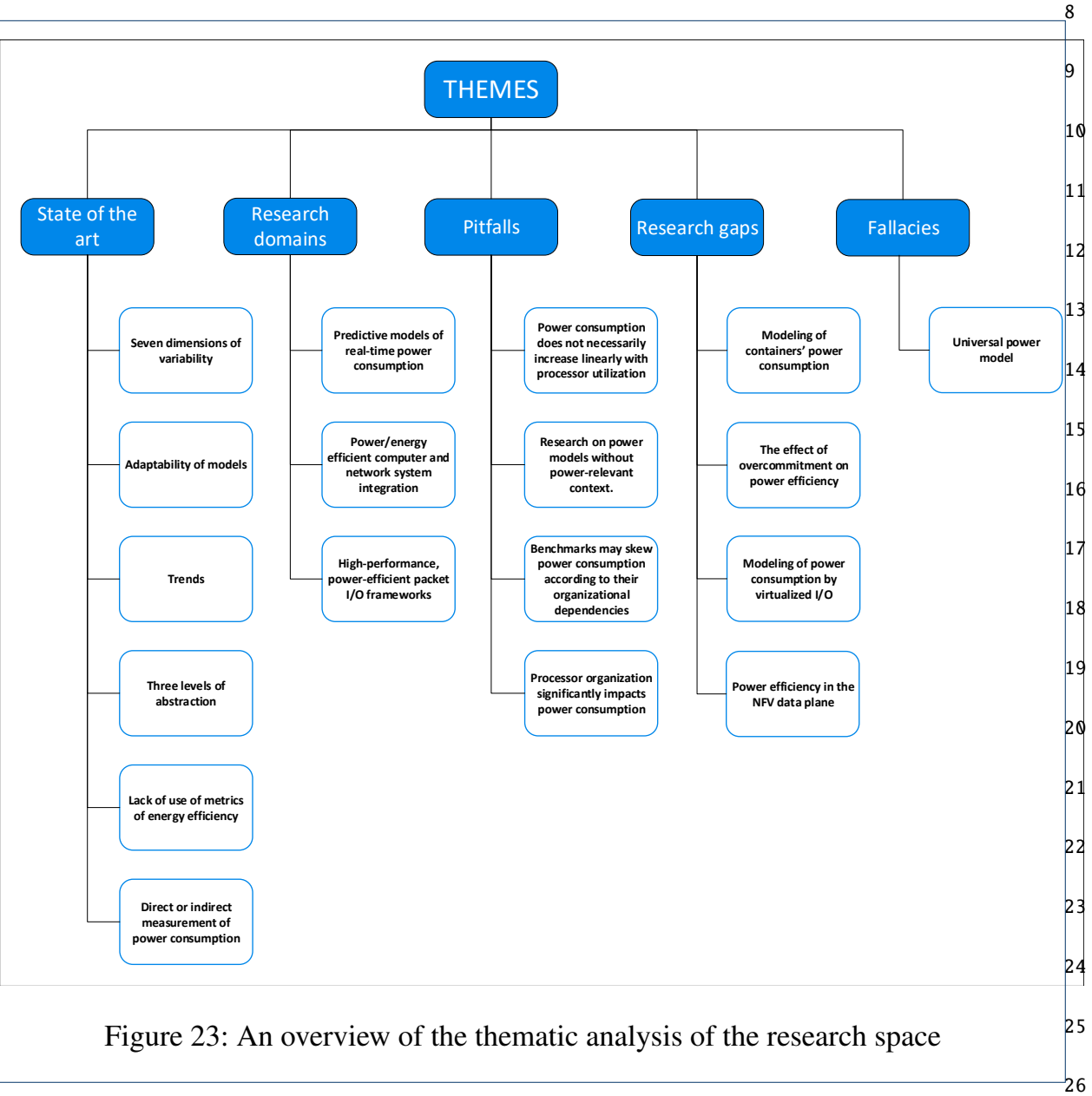




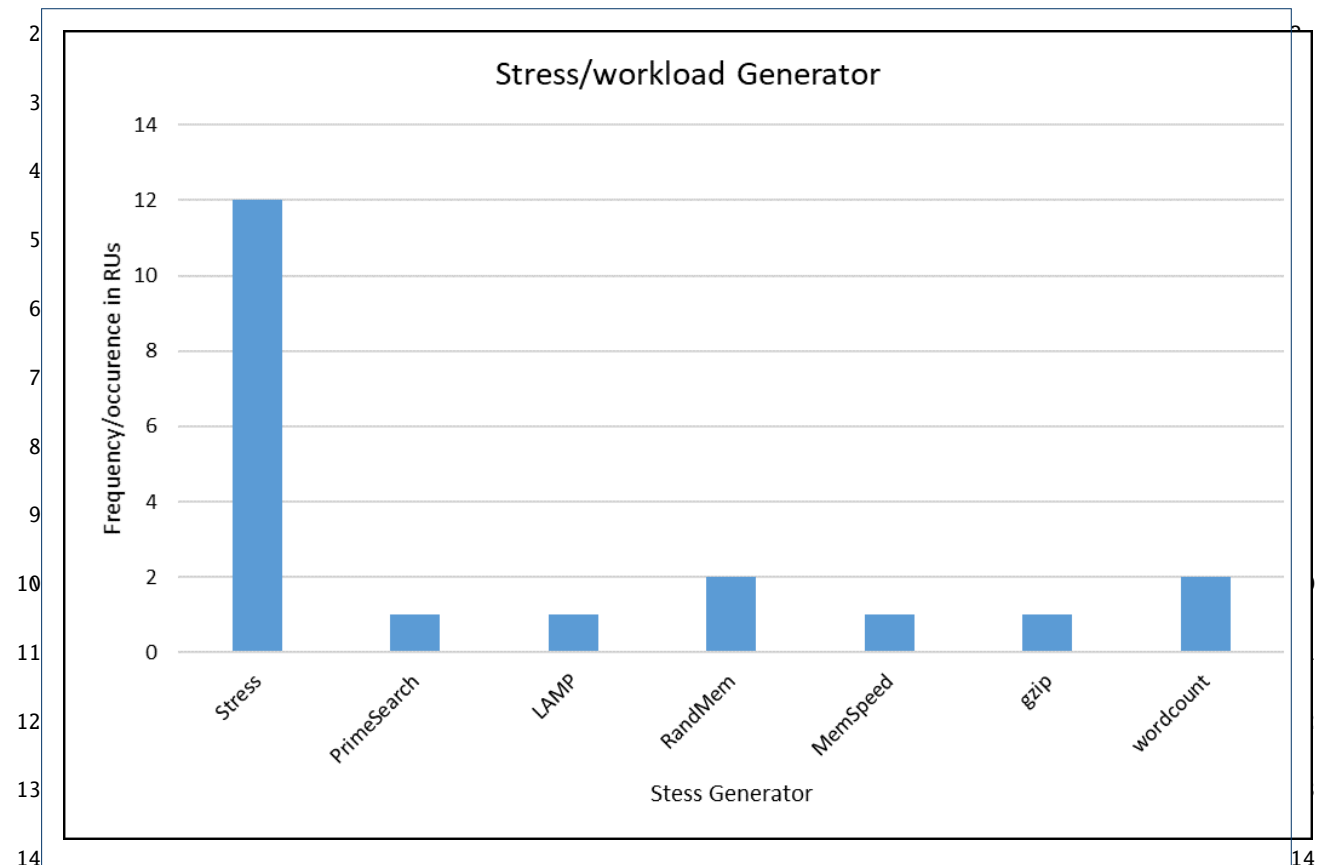

Figure 24: Frequency of stress generator tools used in research studies included in this survey

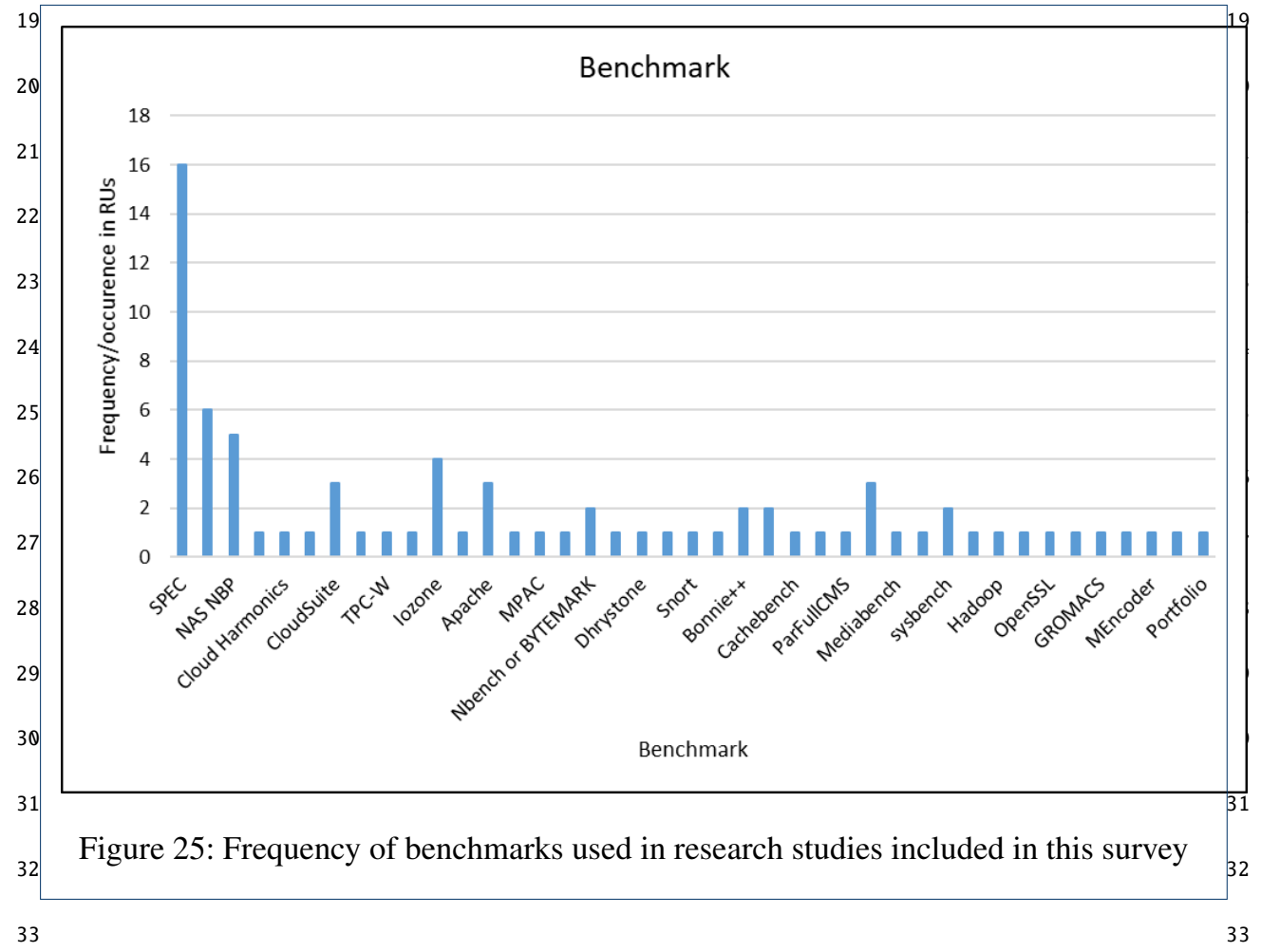




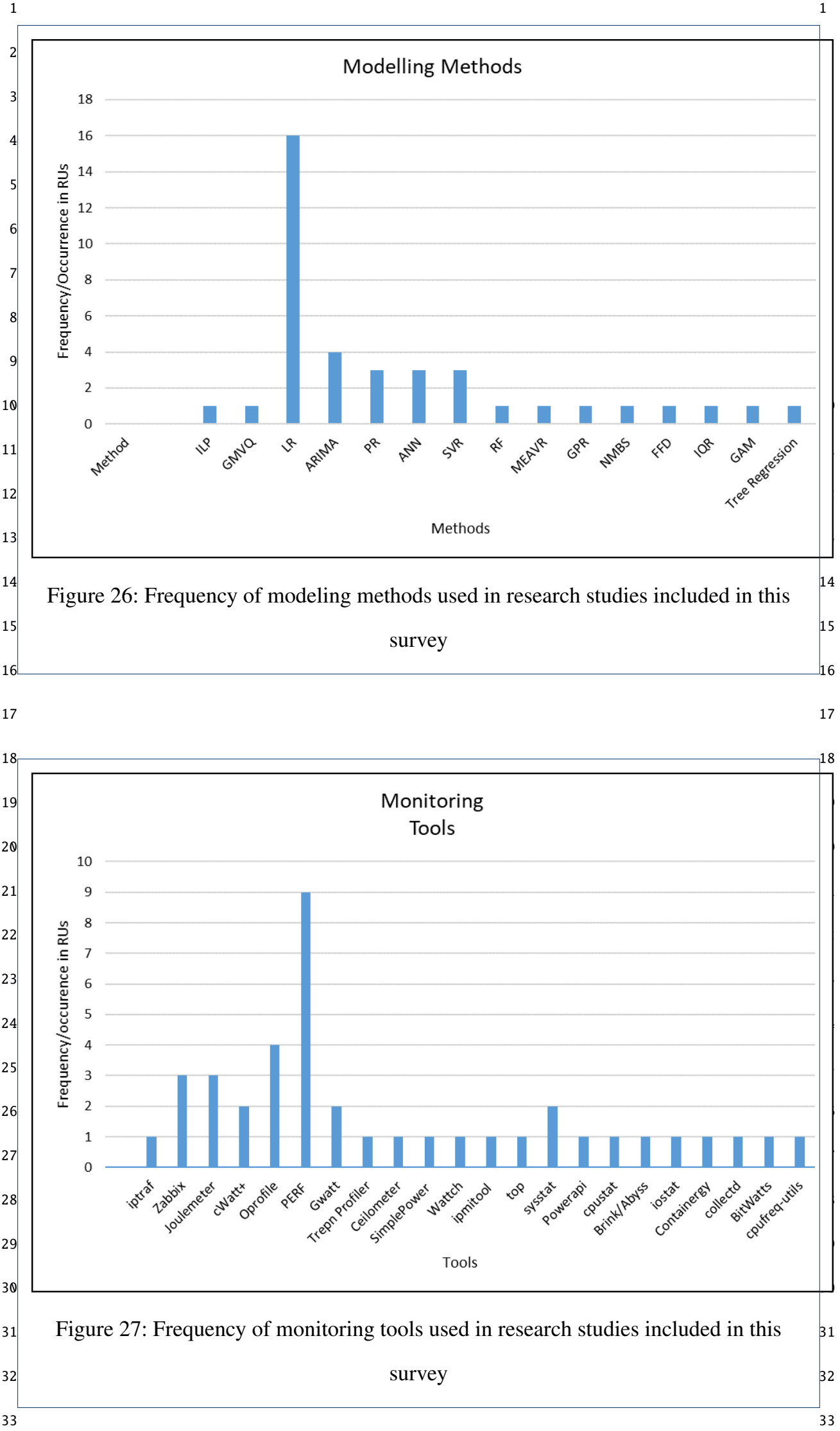

